\title{
Charge and Energy Transport in Disordered Organic Semiconductors
}

\author{
Hassan Abdalla
}

Complex Materials and Devices

Department of Physics, Chemistry and Biology (IFM)

Linköping University, SE-581 83 Linköping, Sweden 
Copyright (C Hassan Abdalla

Charge and Energy Transport in Disordered Organic Semiconductors

ISSN 0345-7524

ISBN 978-91-7685-352-8

Printed by LiU-Tryck. Linköping, Sweden, 2018 
"Flying is learning how to throw yourself at the ground and miss." Douglas Adams 

Improvement of the performance of organic disordered semiconductors (OSC) is driven by the understanding of the underlying charge transport mechanisms and systematic exploitation thereof. There exists a multitude of materials and material systems based on polymers and small molecules with promising performance for use in organic light emitting diodes, photovoltaics, organic field-effect transistors and thermoelectrics. However, universal understanding of many classes of these materials has eluded researchers, due to their broad spectrum of morphologies, molecular structures and electrical properties. Building on the large body of existing models, this thesis deals with charge transport phenomena from the perspective of transport energetics, by studying the interplay between a few but important concepts commonly accepted to play a crucial role in all OSC materials; energetic disorder, charge carrier hopping and Coulomb interactions. The influence of these concepts on the energetic landscape through which charge carriers move and how this translates to experimentally observed transport phenomena are studied by a combination of experimental work, kinetic Monte Carlo (MC) simulations and empirical and analytical models.

The universal scaling and collapse of the temperature and electric field dependence of the conductivity of PEDOT:PSS to a single curve is shown to be functionally equivalent to the scaling of the effective temperature, which describes the effect of field heating as a broadening of the charge carrier distribution. From numerical investigation of the energy relaxation, an empirical model is developed that relates the physical meaning behind both concepts to the heat balance between Joule heating of the carrier distribution via the effective temperature and energy loss to the lattice. For this universal description to be applicable a strongly energy-dependent density of states (DOS) as well as Coulomb interactions and large carrier concentrations are needed.

Chemical doping is a common way of improving charge transport in OSC and is also beneficial for energy transport, which combined leads to an increased thermoelectric power factor. The ensuing thermoelectric investigations not only showed the 
potential of these materials for use in thermoelectric generators but are also helpful in unravelling charge transport mechanism as they give direct insight into the energetics of a material. Interestingly, doped OSC exhibit the same universal powerlaw relationship between thermopower and conductivity, independent of material system or doping method, pointing towards a common energy and charge transport mechanism. In this thesis an analytical model is presented, which reproduces said universal power-law behaviour and is able to attribute it to Variable Range Hopping (VRH) or a transition between Nearest Neighbour Hopping (NNH) and VRH at higher concentrations. This model builds on an existing three-dimensional hopping formalism that includes the effect of the attractive Coulomb potential of ionized dopants that leads to a broadening of the DOS. Here, this model is extended by including the energy offset between host and dopant material and is positively tested against $\mathrm{MC}$ simulations and a set of thermoelectric measurements covering different material groups and doping mechanisms.

Organic field effect transistors (OFETs) have become increasingly comparable in electrical mobility to their inorganic (silicon) counterparts. The spatial extent of charge transport in OFETs has been subject to debate since their inception with many experimental, numerical and analytical studies having been undertaken. Here it is shown that the common way of analysing the dimensionality of charge transport in OFETs may be prone to misinterpretations. Instead, the results in this thesis suggest that charge transport in OFETs is, in fact, quasi-two-dimensional (2D) due to the confinement of the gate field in addition to a morphology-induced preferred in-plane direction of the transport. The inherently large charge carrier concentrations in OFETs in addition to the quasi-2D confinement leads to increased Coulomb interaction between charge carriers as compared to bulk material, leading to a thermoelectric behaviour that deviates from doped organic systems. At very large concentrations interesting charge transport phenomena are observed, including an unexpected simultaneous increase of the concentration dependence and the magnitude of the mobility, the appearance of a negative transconductance, indicating a transition to an insulating Mott-Hubbard phase. The experimental and numerical results in this thesis relate these phenomena the intricacies of the interplay between Coulomb interactions, energetic disorder and charge carrier hopping. 


\section{PUBLICATIONS INCLUDED IN THIS THESIS}

[1] Effective Temperature and Universal Conductivity Scaling in Organic Semiconductors

Hassan Abdalla, Kevin van de Ruit \& Martijn Kemerink Scientific Reports 5, 16870 (2015)

[II] Impact of doping on the density of states and the mobility in organic semiconductors

Guangzheng Zuo, Hassan Abdalla \& Martijn Kemerink Physical Review B 93, 235203 (2016)

[III] Range and energetics of charge hopping in organic semiconductors Hassan Abdalla, Guangzheng Zuo \& Martijn Kemerink Physical Review B 96, 241202(R) (2017)

[IV] Investigation of the dimensionality of charge transport in organic field effect transistors Hassan Abdalla, Simone Fabiano \& Martijn Kemerink Physical Review B 95, 85301 (2017)

[V] I: The Role of Coulomb Interactions in Charge and Energy Transport in Organic Field Effect Transistors Hassan Abdalla, Simone Fabiano \& Martijn Kemerink In review with Physical Review B as of February 2018

[VI] II: Coulomb Interactions at High Carrier Densities in Organic Field Effect Transistors

Hassan Abdalla \& Martijn Kemerink In review with Physical Review B as of February 2018 


\section{PUBLICATIONS NOT INCLUDED IN THIS THESIS}

[I] Two-dimensional charge transport in molecularly ordered polymer fieldeffect transistors Valerio D'Innocenzo, Alessandro Luzio, Hassan Abdalla, Simone Fabiano, Maria Antonietta Loi, Dario Natali, Annamaria Petrozza, Martijn Kemerink and Mario Caironi

Journal of Materials Chemistry C 4, pp 11135-11142 (2016)

[II] Molecular Doping and Trap Filling in Organic Semiconductor Host-Guest Systems

Guangzheng Zuo, Zhaojun Li, Olof Andersson, Hassan Abdalla, Ergang Wang, and Martijn Kemerink.

The Journal of Chemistry C, 121 (14), pp 7767-7775 (2017)

[III] High Thermoelectric Power Factor from Multilayer Solution-Processed Organic Films

Guangzheng Zuo, Olof Andersson, Hassan Abdalla and Martijn Kemerink. Applied Physics Letters, 112(8), 83303 (2018) 


\section{CONTRIBUTIONS TO THE INCLUDED PAPERS}

[I] Performed all simulations and analyzed numerical and experimental results together with the co-authors. Wrote the draft for the manuscript and performed corrections and revisions together with the co-authors.

[II] Provided part of the simulation code and helped analyzed numerical and analytical results. Wrote part of the manuscript draft and revised it together with co-authors.

[III] Built and automated experimental setups, performed all simulations and analytical calculations. Analyzed numerical and experimental results together with the co-authors. Wrote most the draft for the manuscript and performed corrections and revisions together with the co-authors.

[IV] Performed all simulations and analyzed numerical and experimental results together with the co-authors. Wrote most of the draft for the manuscript and performed corrections and revisions together with the co-authors.

[V] Performed all simulations and analyzed numerical and experimental results together with the co-authors. Wrote most the draft for the manuscript and performed corrections and revisions together with the co-authors.

[VI] Performed all simulations and analyzed numerical results together with the co-author. Wrote most the draft for the manuscript and performed corrections and revisions together with the co-author. 


\section{CONTENTS}

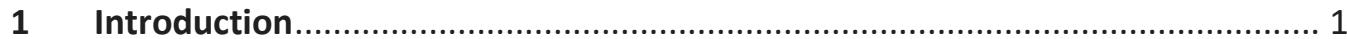

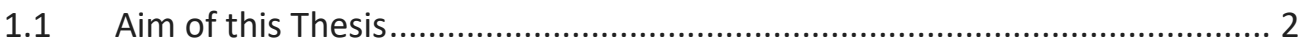

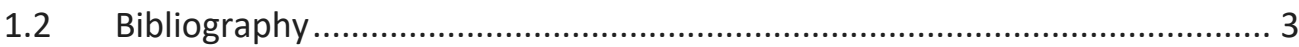

2 Hopping Transport and Energetics ……..................................................... 5

2.1 Density of States and Density of Occupied States....................................... 5

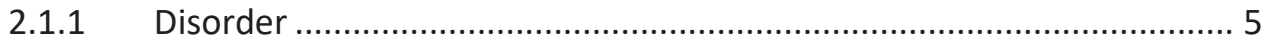

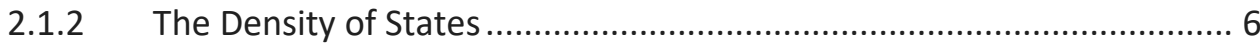

2.1.3 The Density of Occupied States ......................................................... 8

2.1.4 Modifications of the Density of States .............................................. 9

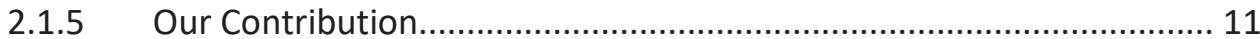

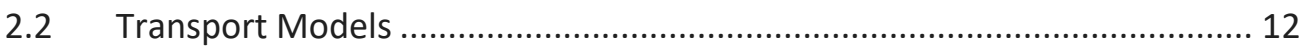

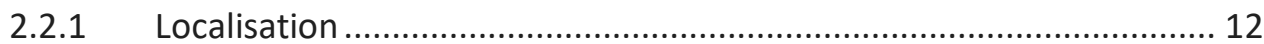

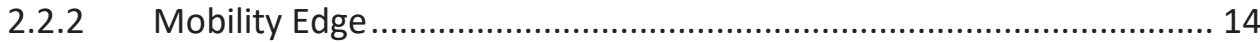

2.2.3 Hopping Transport........................................................................ 15

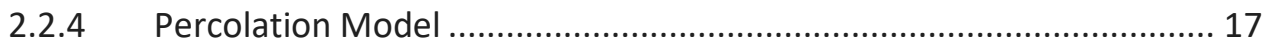

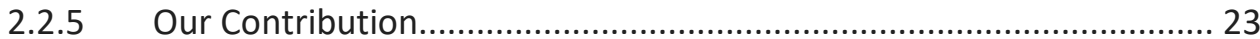

2.2.6 DOS Modification due to Presence of lonized Dopants ...................... 23

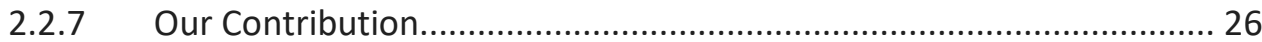

2.2.8 Short Range Carrier-Carrier interaction and Coulomb Gap................. 27

$2.3 \quad$ Non-Ohmic Charge transport ................................................................... 30

2.3.1 The Reduced Activation Energy .......................................................... 30

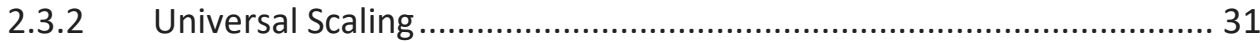

2.3.3 Effective Temperature ................................................................. 33

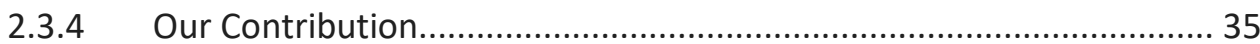




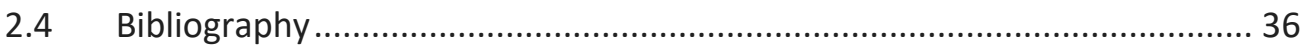

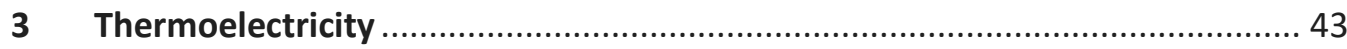

3.1 The Seebeck and Peltier Effect ..................................................................... 43

3.2 Applications of the Seebeck Effect .......................................................... 46

3.3 Thermoelectric Transport Models - Conductivity and Temperature

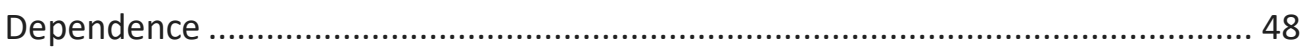

3.3.1 General Expression for the Seebeck Coefficient ................................. 48

3.3.2 Seebeck Coefficient from Statistical Mechanics................................... 50

3.3.3 Seebeck Coefficient from Percolation in a Gaussian DOS .................... 52

3.3.4 Seebeck Coefficient from the Mobility Edge Model............................. 53

3.3.5 Seebeck Coefficient from Mott's Variable Range Hopping Model....... 53

3.3.6 Power-law Relationship between Seebeck Coefficient and Electrical

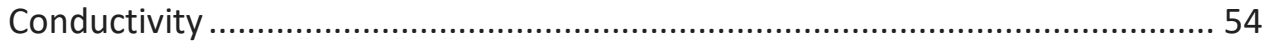

3.3.7 Seebeck Coefficient from a General Transport Function ..................... 55

3.3.8 Our Contribution............................................................................. 56

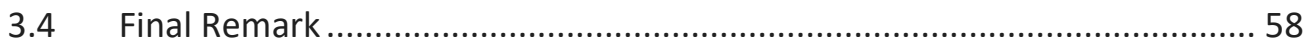

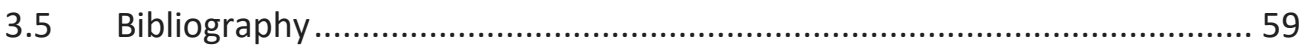

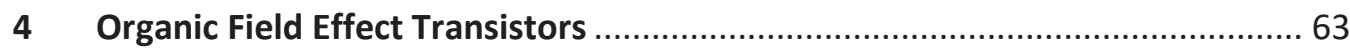

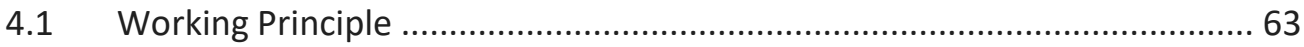

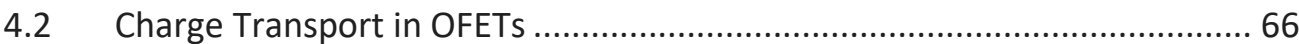

4.2.1 Dimensionality of Charge Transport.................................................. 67

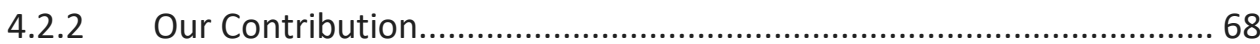

4.3 Concentration dependence of the Mobility ............................................... 70

4.4 Transport at High Relative Concentrations ................................................. 72

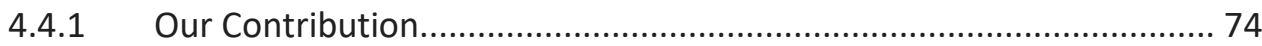

4.5 Thermoelectric properties of OFETs ........................................................... 75

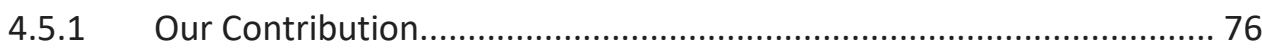

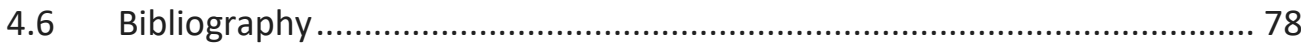




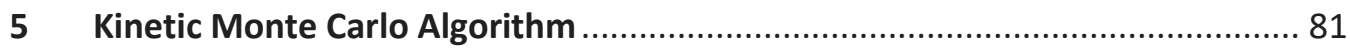

5.1 Geometries and Hopping Mechanism .................................................. 82

5.2 Calculation of Density of States and Density of Occupied States................ 85

$5.3 \quad$ Thermoelectric coefficients ....................................................................... 87

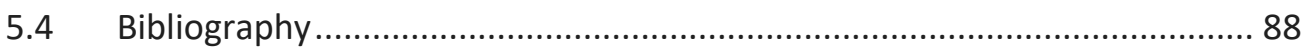

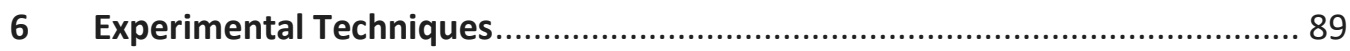

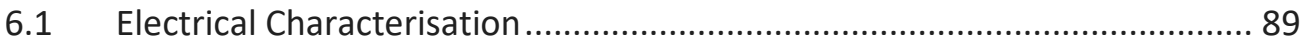

6.2 Determination of the Seebeck Coefficient ................................................. 90

6.3 Determination of the Seebeck Coefficient of OFETs .................................. 93

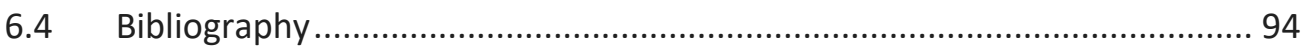

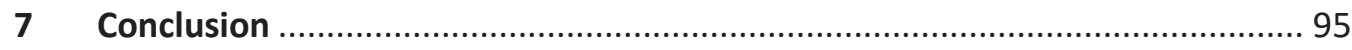

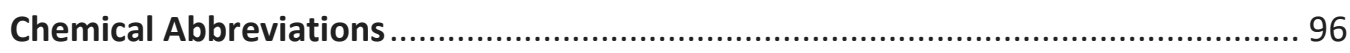

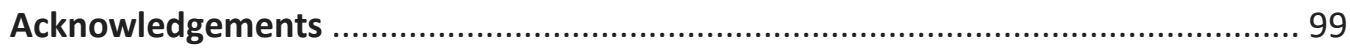




\section{INTRODUCTION}

Organic disordered semiconductors (ODSCs) have shown great potential for replacing inorganic materials in applications such as thin film transistor and photovoltaics and are in fact already commercialised in the form of organic light-emitting diodes. The main arguments in favour of ODSCs are that they are solution-processible from cheap, readily available materials at a very low energy expenditure and can be used in all manners of flexible applications. Since the start of this thesis work, the field of organic thermoelectrics has seen an especially steep increase in research activity. Some classes of ODSCs, such as PEDOT derivatives, have shown promising potential as thermoelectric materials due to their high electrical conductivities combined with the inherently large Seebeck coefficient and low thermal conductivity ${ }^{1,2}$ (the fundamentals of thermoelectricity are discussed in section 3 ). Due to the chemical instability beyond $200^{\circ} \mathrm{C}$, possible use for organic thermoelectric generators are limited to room temperature applications such as powering small devices from body heat by integrating them into clothing, to name one example.

However, the direct connection between Seebeck coefficient, which can be measured relatively easy (see section 6), and energetic conditions in a material, make thermoelectric investigations a simple but powerful tool in the unravelling and understanding of charge and energy transport in the great variety of ODSCs. Thus far, investigations of this kind have revealed interesting connections between morphology, energetic disorder, conductivity and Seebeck coefficient, such as a wide range of doped organic semiconductors following the same empirical relationship between Seebeck coefficient ${ }^{3-5}$ and the absence of this trend in organic field-effect transistors (OFETs) ${ }^{6,7}$. The quasi-universal trend of doped systems cannot be explained by common charge transport models that were otherwise successful in describing temperature and concentration dependence of electrical mobility and conductivity (some of these models are described in section 2) and thus highlights the need for new insights into charge transport and models that consider the energetic interactions in the material. 
OFETs have also demonstrated significantly improved of performance over the last decade, while recently also exhibiting new charge transport phenomena as carrier concentrations approach those of molecular sites in the material. At such concentrations carrier-carrier Coulomb interactions become dominant, crucially effecting the charge transport characteristics. Hence also here exists a need for the inclusion of energetic interactions into charge transport models, as a recently published work demonstrated $^{8}$.

In general, charge transport at high concentrations leads to a variety of scientifically interesting effects that may also be controlled and used to an advantage if the appropriate understanding exists. Thermoelectric investigations in conjunction with electrical characterisations are thus an excellent way of achieving a deeper understanding

\subsection{AIM OF THIS THESIS}

The work conducted in this thesis was aimed at gaining a deeper understanding of the charge and energy transport mechanisms, by combining a previously developed kinetic Monte Carlo (MC) algorithm with thermoelectric and electrical characterisation. A special focus was set on the investigation of transport phenomena at play predominantly under the assumption of high charge carrier concentrations compared to those found in organic solar cells or light-emitting diodes. Hence, the results are applicable to the situations found in organic field-effect transistors or highly chemically doped polymer materials, such as those exhibiting good thermoelectric behaviour. Under these conditions inter-carrier Coulomb interactions as well as the effect of carriers on their environment play a crucial role to charge transport and are, in fact, the red thread that connects the published works of this thesis.

The kappa of this thesis should create the context in which the thesis is placed and offer brief introductions to the topics treated in the scientific part of the thesis. Most subsections include a section called "Our Contribution" that highlights the work of this thesis within context of the subsection. Chemical abbreviations are found at the end of the kappa. 


\subsection{BIBLIOGRAPHY}

1. Bubnova, O. et al. Optimization of the thermoelectric figure of merit in the conducting polymer poly(3,4-ethylenedioxythiophene). Nat. Mater. 10, 429433 (2011).

2. Bubnova, O. et al. Semi-metallic polymers. Nat. Mater. 13, 190-4 (2014).

3. Kaiser, A. B. Electronic transport properties of conducting polymers and carbon nanotubes. Reports Prog. Phys. 64, 1-49 (2001).

4. Glaudell, A. M., Cochran, J. E., Patel, S. N. \& Chabinyc, M. L. Impact of the Doping Method on Conductivity and Thermopower in Semiconducting Polythiophenes. Adv. Energy Mater. 5, 1401072 (2015).

5. Russ, B., Glaudell, A., Urban, J. J., Chabinyc, M. L. \& Segalman, R. A. Organic thermoelectric materials for energy harvesting and temperature control. Nat. Rev. Mater. 1, 16050 (2016).

6. Pernstich, K. P., Rössner, B. \& Batlogg, B. Field-effect-modulated Seebeck coefficient in organic semiconductors. Nat. Mater. 7, 321-325 (2008).

7. Venkateshvaran, D. et al. Approaching disorder-free transport in high-mobility conjugated polymers. Nature 515, 384-388 (2014).

8. Di Pietro, R. et al. Coulomb Enhanced Charge Transport in Semicrystalline Polymer Semiconductors. Adv. Funct. Mater. 26, 8011-8022 (2016). 



\section{HOPPING TRANSPORT AND ENERGETICS}

It is mostly assumed that the transport of charge carriers, and thus electrical conduction, in organic disordered semiconductors (ODSC) occurs via thermally activated tunnelling between energetically localised sites, commonly referred to as hopping. In this chapter the physical process of hopping is discussed as well as the properties and distribution of hopping sites, i.e. the energetic landscape on which hopping occurs. Several widely-employed hopping transport models and formalisms are discussed in this chapter, focussing on those relevant to the scientific work of this thesis.

\subsection{Density of States And Density of Occupied States}

The overlap of $p_{z}$ orbitals in $\pi$-conjugated carbon atoms gives rise to the formation of molecular orbitals, where the number of available orbital energies scales with the number of conjugated carbon in the system. A large number of conjugated atoms would give rise to a metallic material with no gap between the highest occupied and lowest unoccupied state. Peierls distortion, the alternation of bond lengths between conjugated atoms, however, leads to the formation of a band gap, the extent of which depends on the difference between large and small bond lengths ${ }^{1}$.

\subsubsection{Disorder}

For charge transport, typically only the highest occupied molecular orbital (HOMO) or the lowest unoccupied molecular orbital (LUMO) is responsible for the conduction of holes and electrons, respectively. In reality, ODSC materials consist of many polymer chains of finite and different lengths, exhibiting kinks, twist, torsion and other conformational defects that break up the uninterrupted pi-conjugation of the ideal polymer chain into smaller $\pi$-conjugated chain segments. Within the context of this thesis such an element on which conjugation is, to a large degree, uninterrupted forms an energetic site (or more precisely a site is a collection of site energies that are so close together in energy and real space that they become virtually indistinguishable w.r.t the other energy scales in the system such as the thermal 
energy). The energetic disorder between sites depends on the conformation of the polymers and their orientation with respect to each other. The energy difference between site energied in ODSCs is typically large enough to create localized sites where the wavefunctions of charge carriers on neighbouring sites do not overlap significantly (see section 2.2.1). Hence transport between sites is governed by (thermally activated) tunnelling between them.

Further disorder is introduced by the morphology of the whole polymer material. Crystallinity, polymer aggregation, dopants as well as the presence of any charged species all have an effect on the energetic landscape of a material. Morphologically induced disorder is often correlated in space. The energetic disorder is thus a collective property of morphology, chemical structure, constituent atoms and processing condition of an ODSC, rather than inherent to a particular polymer. Importantly, the energetic disorder can in general not simply be inferred purely from the morphology ${ }^{2}$.

\subsubsection{The Density of States}

Information about the energetic distribution of localised sites in a material is contained in the density of states (DOS) $g(E)$, which is defined as the number of states $N$ per volume within an energy interval $\Delta E$ that is available for occupation by a charge carrier,

$$
g(E)=\frac{\text { Number of sites }}{\text { Volume } \cdot \text { Energy }}=\frac{N}{\Delta E}
$$

The mathematical description of the energetic disorder is crucial to the development of models that describe charge transport in ODSC. However, several decades after the first description of organic semiconductors the mathematical shape of the DOS is still subject to debate ${ }^{3}$. For amorphous inorganic semiconductors ${ }^{4}$ and in organic semiconductors in field-effect transistors ${ }^{5}$, typically an exponential distribution of site in energy $E$ is assumed following

$$
g(E)=\frac{N_{\text {site }}}{k_{B} T_{0}} \exp \left(\frac{E}{k_{B} T_{0}}\right)
$$

where $N_{\text {site }}$ is the concentration of localised sites, $T_{0}$ is the characteristic temperature of the distribution (related to the disorder) and $k_{B}$ is the Boltzmann constant. A Gaussian distribution is believed to be the case for many bulk organic semiconductors ${ }^{6}$ and takes the form 


$$
g(E)=\frac{N_{\text {site }}}{\tilde{\sigma} \sqrt{2 \pi}} \exp \left(\frac{E^{2}}{2 \tilde{\sigma}^{2}}\right),
$$

where $\tilde{\sigma}$ is the standard deviation (disorder) of the Gaussian DOS. For systems with a very large disorder a constant DOS can be assumed. Figure 2.1 schematically depicts how the site distribution relates to the DOS with the example of a Gaussian distribution.

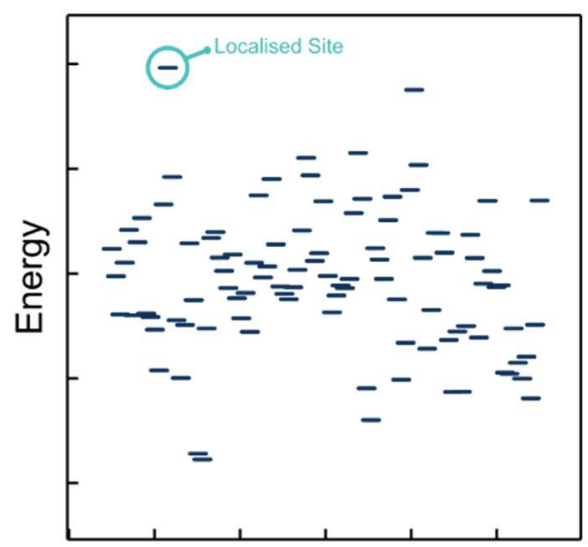

Space

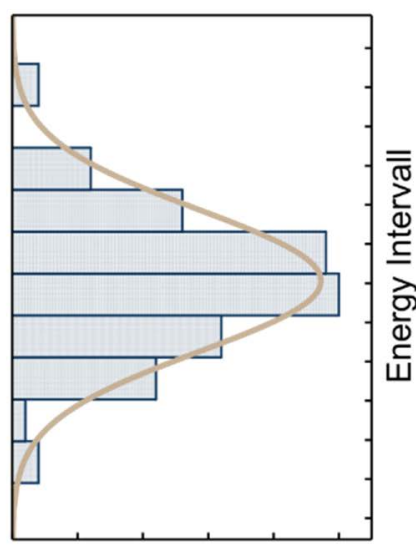

Density of States

Figure 2.1 Distribution of localised sites in space and energy according to a Gaussian distribution Equation (2.3)

There is experimental evidence in favour of both distributions indicating that the shape of the DOS might be dependent not only on the chemical properties of the material, but also its use (e.g. as an active layer in an organic light-emitting diode (OLED) vs. an organic field-effect transistor (OFET)), carrier concentration and processing conditions. The work in this thesis aligns with this opinion and will treat different materials in different device constructions with a DOS that matches previously established opinions in literature as well as own experimental results. However, to extend generality all applied models, numerical or otherwise, have been checked against the "other" DOS shape to see which one delivers the better and more appropriate description of experiments and established charge transport physics. The width of the DOS, irrespective of the shape, varies widely, but ranges in general between 30-50 meV for crystalline and semi-crystalline materials such as P3HT, DPDTTT $^{2}$ and reach up to more than $100 \mathrm{meV}$ for PEDOT:PSS, for which often a constant DOS is assumed. 


\subsubsection{The Density of Occupied States}

Unless stated otherwise, this thesis deals predominantly with the transport of holes, rather than electrons, however, few fundamental differences in transport properties have been found so far. Thus, conduction is facilitated in the HOMO DOS that is usually populated by charges transferred by electron accepting dopant materials (see Papers I, II, III) or electrostatically by an electric field at the semiconductor-dielectric interface of a field-effect transistor (see section 4 and Papers IV, V, VI).

In this thesis mainly high charge carrier concentrations $\geq 1 \%$ are concerned in a temperature range from around $5 \mathrm{~K}$ to around $400 \mathrm{~K}$ both experimentally and theoretically. Furthermore, the interaction of charge carriers with each other in the material are paramount to the discussions and elaborations presented here. As such, Boltzmann statistics, often used in the description of OLED and organic photovoltaics (OPV) due to the low carrier concentrations involved, are not considered here.

Rather, the distribution of charge carriers in the DOS is considered to be according to the full Fermi-Dirac statistics. In this framework the density of occupied states (DOOS), which is the distribution of charges in energy, is given by the product of the DOS in Equation 2.2 or 2.3 and the Fermi-Dirac distribution,

$$
f(E)=\frac{1}{e^{\frac{E-E_{F}}{k_{B} T}}+1},
$$

where $E_{F}$ is the Fermi level and $T$ is the temperature. Inversely, one can also obtain the Fermi level and the temperature by fitting the Fermi-Dirac distribution to the quotient of DOOS and DOS. This procedure is used for numerical modelling as further described in section 5 . The Fermi level $E_{F}$ is the energy at which the probability of occupying a localised site by a charge carrier at the temperature $T$ is $50 \%$. In an exponential DOS $E_{F}$ is usually close to the peak of the DOOS, however, in a Gaussian DOS at lower carrier concentrations, this is no longer the case due to the more-thanexponential increase of the energy dependence of the DOS. Most carriers are then located at the so-called equilibrium energy ${ }^{6}$ and transport is usually considered to be activated from the concentration independent energy $E_{\infty}=\hat{\sigma}^{2} / k_{B} T$ to some transport energy ${ }^{3}$. However, as stated before, the work in this thesis considers relatively high concentrations where $E_{F}$ is larger than $E_{\infty}$ for the disorders and temperature ranges used. 
The DOS is notoriously hard to determine experimentally. Indirectly, one can make statements about the shape of the DOS by comparing the charge carrier concentration dependence from analytical and numerical models with experimental results. A much-used example to show the difference in charge transport between exponential and Gaussian distribution is the determination of the charge carrier concentration dependence of the mobility ${ }^{3,7}$. Due to the independence of $E_{\infty}$ on charge carrier concentration (at lower concentrations) the mobility shows no dependence on the concentration for a Gaussian DOS, whereas an exponential dependence of the mobility is found for an exponential DOS (due to the state-filling) ${ }^{5}$. However, it has been argued that the exponential DOS is a good approximation of the tail of the Gaussian DOS ${ }^{8}$. This is advantageous because analytical solutions to transport equations can be found for exponential DOS, whereas, due to the nature of Equation 2.3, a closed analytical solution cannot be found for a Gaussian DOS for many transport models, without simplifications. One method to directly determine the width of the DOS is from the Urbach energy, which can be extracted from absorbance or thermal deflectance measurements ${ }^{2}$.

\subsubsection{Modifications of the Density of States}

Further complicating the determination of the DOS is the fact that it is subject to change with charge carrier concentration ${ }^{9-11}$. The Coulomb interactions between charge carries put an energy penalty on sites close to a charge carrier in addition to the original energy distribution from Equations (2.2) and (2.3).

This modification of the DOS has an appreciable influence on charge transport and energetics if it is larger than the $k_{B} T$, the thermal energy available to charge carriers. The sphere in space where these two energies are equal and within which modifications matters most, is characterized by the Coulomb interaction radius,

$$
r_{C}=\frac{q}{4 \pi \varepsilon_{0} \varepsilon_{r} k_{B} T} .
$$

Consequently, the DOS will not retain its form from Equations (2.2) or (2.3) but will broaden and change shape as carriers fill up the system. A numerically obtained (see section 5) example of this can be seen in Figure 2.2 which depicts the transformation of an initially exponential DOS to a broader and more Gaussian shape with increasing charge carrier density, where charge carriers are introduced electrostatically. Charge carriers can also occupy a site that is already occupied by paying the Coulomb energy 
penalty associated with overcoming the on-site barrier. The possibility of multiple occupation gives rise to an additional "DOS" following size and shape of the DOOS and shifted in energy by the on-site repulsion energy, which is clearly visible in Figure 2.2 above approximately $10 \%$ concentration. Also, the DOOS will broaden and shift to higher energies since charge carriers want to avoid each other. The extent to which Coulomb interactions influence shape and width of the DOS depends on the interplay between inter-carrier Coulomb interactions and on-site barrier and takes different shapes for different ratios between these two.

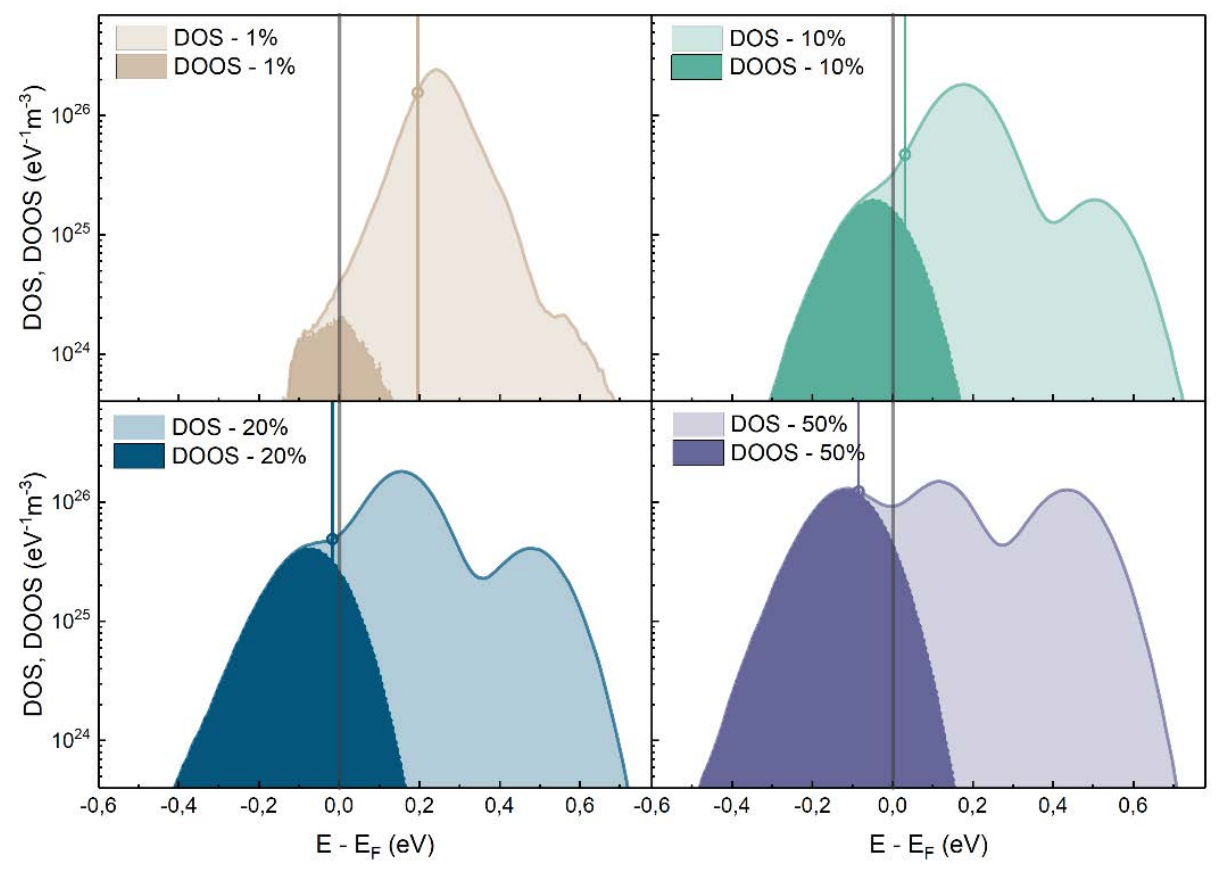

Figure 2.2 Modification of an initially exponential DOS (light shaded area) and corresponding DOOS (filled area) with increasing charge carrier concentration from $1 \%$ per-site concentration (top left) to $50 \%$ (bottom right). The DOS and DOOS are plotted as a function of energy with respect to the Fermi level. The vertical solid coloured lines indicate the position of the transport energy (see sections 2.2.4.2 and 5). DOS and DOOS have been numerically obtained using the kinetic Monte Carlo algorithm described in section 5 and take into account full Coulomb interactions between charge carriers and the effect on unoccupied sites.

The Coulombic influence of charged species such as dopant ions on the DOS is discussed separately in sections 2.2 .6 and 2.2.7. 


\subsubsection{Our Contribution}

In Papers V and VI we have shown that the changes to DOS and DOOS as well as increased Coulomb scattering between charge carriers have a strong effect on charge and energy transport of OFETs and are, in fact, crucial to the numerical reproduction of experimentally observed phenomena at very high concentrations of around $100 \%$ site occupation.

The attractive potential of immobile ions introduced by chemical doping acts as a trap for mobile charge carriers, which manifests itself in the development of an exponential tail and a broadening of the main DOS as, explained in section 2.2.6. In Paper II we have developed an analytical model that describes charge transport in the dopant-modified DOS and find that the analytical results reproduce well numerical simulations and mobility measurements of $\mathrm{F}_{4} \mathrm{TCNQ}$-doped P3HT.

The effects of the presence of dopant ions on thermopower are studied in Paper III, where it was also found to be crucial in reproducing the experimentally observed quasi-power-law relationship between thermopower and electrical conductivity (see section 3.3.6).

Additionally, the combined findings of Papers II, III, V and VI show that the question of which disorder to use depends very much on the charge carrier concentration and may deviate significantly in shape and width from the DOS at low concentrations. 


\subsection{TRANSPORT MODELS}

This section gives a concise overview of commonly employed models for describing charge transport in organic disordered semiconductors and connecting the DOS to charge transport properties.

\subsubsection{Localisation}

The localisation of energetic states has been treated by Anderson (initially for amorphous inorganic semiconductors) by considering a three-dimensional (3D) array of quantum wells with coordination number $z$. Their depths are randomly distributed in energy leading to a Gaussian distribution characterised by a width $V_{0}$ around a mean energy $E_{M}$, depicted schematically in Figure $2.3^{12}$.

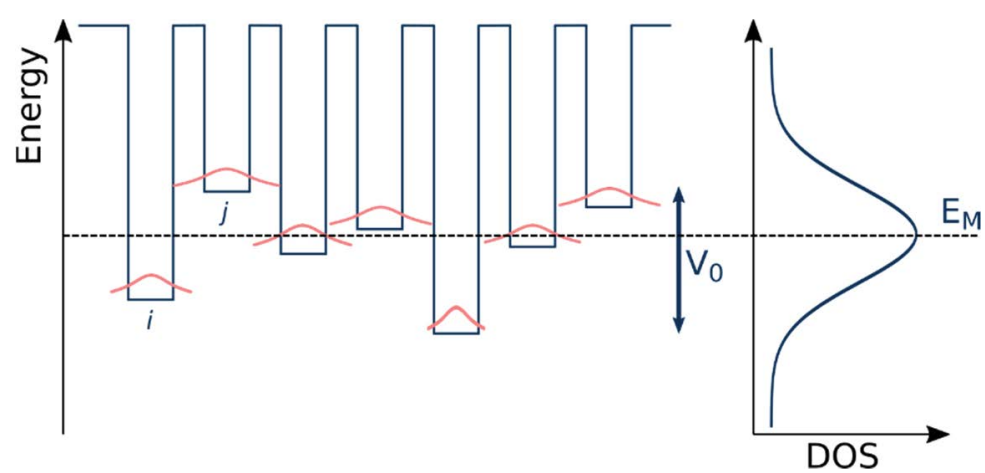

Figure 2.3 Schematic depiction of Anderson localisation due to a Gaussian distribution of quantum well depths. Red lines indicate ground-state wavefunctions.

The wave function $\psi_{i}$ of a charge carrier trapped in a quantum well $i$ decays exponentially with distance $x$ according to

$$
\psi_{i}(x)=\left(\sqrt{\pi} \alpha^{-\frac{3}{2}}\right)^{-1} \exp \left(-\frac{x-x_{i}}{\alpha}\right),
$$

where $x_{i}$ is the position of the localised site and $\alpha$ is the localization length, i.e. the distance at which the wave function has decayed to $1 / e$ of its original value. For $V_{0}=$ 0 , all wells have the same depth and the system shows no disorder. In this case the overlap of the wave function creates a band with the band width 


$$
B=2 z J,
$$

where $J$ is the transfer integral (sometimes also referred to as overlap integral) between quantum wells (i.e. site energies) $i$ and $j$ and is given by

$$
J_{i j}=\left\langle\psi_{i}|H| \psi_{j}\right\rangle \text {. }
$$

The Hamilton operator $H$ is taken to be

$$
H=-\frac{\hbar^{2}}{2 m} \nabla^{2}-\frac{q^{2}}{4 \pi \varepsilon_{0} \varepsilon_{r} r},
$$

with $m$ the charge carrier mass, $q$ the elementary charge and $\varepsilon_{0}$ the vacuum permittivity. The last term on the right-hand side of equation (2.9) corresponds to the Coulomb interaction between two charge carriers on neighbouring sites, separated by the distance $r$. The magnitude of the transfer integral between two hydrogen-like $1 \mathrm{~s}$ wavefunctions instead of two potential wells is plotted in Figure 2.4 as a function of localization length $\alpha$ and inter-site distance $a_{N N}$.

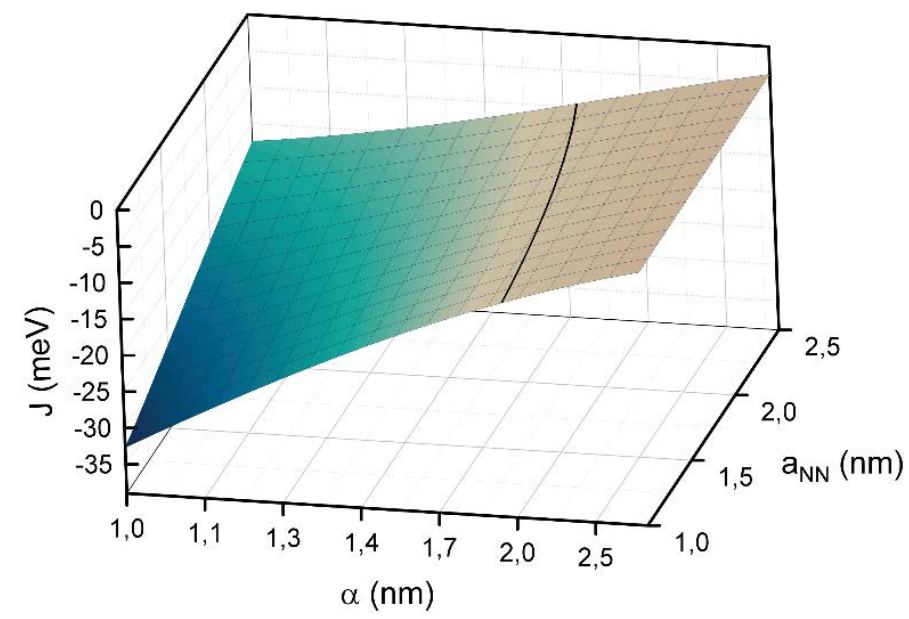

Figure 2.4 Dependence of the transfer integral of two 1s wave functions with localization length $\alpha$ and separated by the distance $a_{N N}$. The black line serves as an approximate limit above which all states are localised (at $V_{0} / B=1.6$ according to reference ${ }^{13}$ ) in a system with a Gaussian disorder of $75 \mathrm{meV}$.

It is intuitive that, as $V_{0}$ increases, the width of the DOS increases and spreads out the sites in energy space and leading to fewer sites with similar energies and less overlap. The transfer integral is proportional to the localization length (Equations (2.6) and 
(2.8)), which itself is proportional to the potential depth and mass of the charge carrier. Hence $J$ decreases with increasing $V_{0}$, as can be seen in Figure 2.4. The transfer integral is also proportional to the spatial separation $r$ between the quantum wells. However, in this simple case there is assumed to be no correlation between the position in space and $V_{0}$ and thus, $r$ is considered constant when changing $V_{0}$. It has been shown in the original work by Anderson that there exists a limit for $V_{0}$ w.r.t to the band width $B$ beyond which all states are localized. The critical ratio for total localization was later found to be $V_{0} / B=1.6$ from numerical calculation ${ }^{13}$. The threshold for complete localisation of the DOS assuming a coordination number of 6 and a disorder potential corresponding to the full width half maximum of a Gaussian distribution with a width of $75 \mathrm{meV}$ is plotted as a black solid line in Figure 2.4. Since the localization length and the average nearest neighbour distance are not necessarily accessible experimentally (or at the very least subject to interpretation) these values have been chosen to the best knowledge throughout the analytical and numerical work in this thesis and lie within the localised regime on the right-hand side of the solid line in Figure 2.4 with $\alpha$ ranging from $0.5 \mathrm{~nm}$ to $2 \mathrm{~nm}$ and $a_{N N}$ ranging from 1 $\mathrm{nm}$ up to $15 \mathrm{~nm}$ (see Paper I-VI). Furthermore, it should be mentioned that complete localisation of the DOS is not a necessary criterion for incoherent hopping to be the dominant charge transport mechanism, as long as the typical transport energies are below the so-called mobility edge, if it exists, that separates localised and delocalised states (see Figure 2.5).

\subsubsection{Mobility Edge}

In an appropriate DOS all sites that sit below a certain energy, the so-called mobility edge $E_{M E}$, are considered to be localised and act as traps for charge carriers, while above $E_{M E}$ all states are delocalized (extended) and form a continuous band in which charge transport is only disturbed by scattering. At high enough concentrations the Fermi level is close enough to the mobility edge and electrical conduction occurs by thermal activation of carriers from $E_{F}$ to $E_{M E}$. During band transport through the material, charge carriers experience multiple trapping events in the lower part of the DOS, from which they need to be (thermally) activated above $E_{M E}$ again ${ }^{14,15}$. This type of transport is typically referred to as the multiple trapping and release (MTR), which is schematically depicted in Figure 2.5 


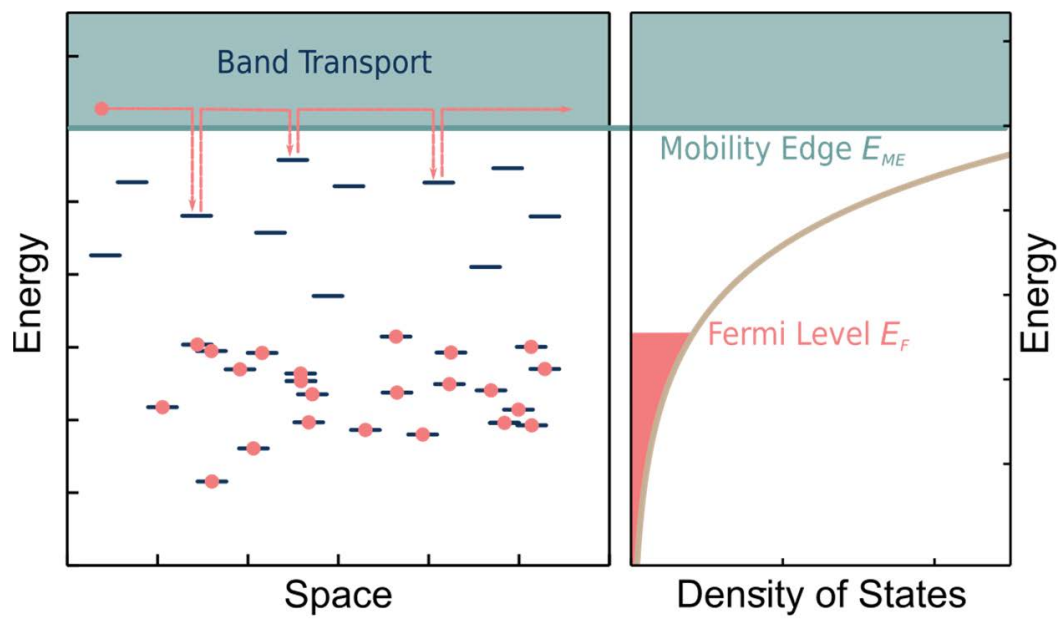

Figure 2.5 Schematic of multiple trapping and release transport mechanism.

Due to the comparatively large disorder of organic materials, the ratio of localised to extended states will be somewhat in favour of the former making these trapping events more likely. Description of the transport will then be more in analogy to conventional hopping models ${ }^{16-18}$, such as the transport energy model (section 2.2.4.2). In materials where ME transport is believed to occur, however, it is likely that band transport occurs on chain segments of the polymer or in the crystalline regions, where the backbone is uninterrupted and the $\pi$-orbitals and the wavefunctions of the occupying charge carriers can delocalize more, while trapping will mostly occur at conformational defects separating chain segments or in the amorphous phase of semi-crystalline material ${ }^{19}$. The MTR model describes the conductivity as the conductivity of the band transport modified by the temperature and material dependent probability of thermal release. This formalism has been shown, in various different forms, to, at least phenomenologically, describe the temperature and field dependence of the mobility in OFETs with semi-crystalline or crystalline materials such as P3HT and PQT-12, with an exponential ${ }^{18,20,21}$ or Gaussian ${ }^{17,22}$ distribution of trap states.

\subsubsection{Hopping Transport}

Thermally activated tunnelling of charge carriers between localised sites was simultaneously suggest by several researchers to describe transport processes in amorphous inorganic semiconductors ${ }^{23-25}$. Due to the large disorder in most organic 
materials and the resulting localization of states, it is nowadays considered that charge transport in ODSC occurs by incoherent (there is no apparent correlation between one hop and the next) tunnelling between localized sites, referred to as hopping. The hopping process between localised sites is thermally activated with hopping rates between two sites $i$ and $j$ expressed either by the Marcus formalism ${ }^{26}$ or, as is done throughout the work of this thesis, by the Miller Abrahams (MA) expression, which follows to 27,28

$$
\vartheta_{i j}=\vartheta_{0} \exp \left(-\frac{2 r_{i j}}{\alpha}-\frac{\left|E_{i}-E_{j}\right|+\left|E_{i}-E_{F}\right|+\left|E_{j}-E_{F}\right|}{2 k_{B} T}\right),
$$

where $r_{i j}$ is the spatial separation of the sites with energies $E_{i}$ and $E_{j}$. The materialdependent prefactor $\vartheta_{0}$ is often referred to as the attempt-to-hop frequency and its magnitude are quite a subject to discussion. Typical values are assumed to be close to the phonon frequency ranging from $10^{10} \mathrm{~s}^{-1}$ to $10^{12} \mathrm{~s}^{-1}$ (see the methods descriptions in Papers I-VI). The first term of the exponent in Equation (2.10) describes the dependence of the tunnelling rate on the spatial distribution of sites and their wavefunction overlap, while the second term relates the energy difference between the two sites as well as between the sites and the Fermi level $E_{F}$ to the hopping probability. However, the hopping rate-limiting steps occur only when hopping from an occupied site of lower energy to an unoccupied site of higher energy, such as depicted in Figure 2.6. Hops downward in energy only suffer a penalty from their spatial separation and so do "hops" between two unoccupied or two occupied sites and are therefore not connected to a penalty in hopping rate and Equation (2.10) takes the form

$$
\vartheta_{i j}=\vartheta_{0} \exp \left(-\frac{2 r_{i j}}{\alpha}\right) \begin{cases}\exp \left(-\frac{E_{j}-E_{i}}{k_{B} T}\right) & \text { for } E_{j}>E_{i} \\ 1 & \text { for } E_{j} \leq E_{i},\end{cases}
$$

which is the form typically encountered in literature and also throughout the scientific work of this thesis. In Papers I, II, IV, V and VI, an electric field $\vec{F}$ is applied in the kinetic Monte Carlo (kMC) simulations to induce directed charge flow in the virtual sample. As will be described in more detail in section 2.3.3, if a positive charge carrier moves a distance $x$ along the direction of the field $\vec{F}$ (or against the field in case of a negative charge), it gains energy, which reduces the energy difference $E_{j}-E_{i}$ perceived by the charge carrier by an amount $q \vec{x} \vec{F}$ and increases its hopping 
probability. Analogously, the difference in Coulomb potential between two sites increases the energy barrier for hopping by

$$
\Delta E_{C}=\frac{q^{2}}{4 \pi \varepsilon_{0} \varepsilon_{r} d}
$$

where $\varepsilon_{0}$ is the vacuum permittivity, $\varepsilon_{r}$ is the dielectric constant of the material and $d$ is the separation between two charge carriers. Including both these effects in Equation (2.11) results in the hopping probability

$$
\vartheta_{i j}=\vartheta_{0} \exp \left(-\frac{2 r_{i j}}{\alpha}\right)\left\{\begin{array}{l}
\exp \left(-\frac{E_{j}-E_{i}-q \vec{x} \vec{F}+\Delta E_{C}}{k_{B} T}\right)=\exp \left(-\frac{\Delta E}{k_{B} T}\right) \Delta E>0 \\
1 \quad \\
\Delta \mathrm{E} \leq 0 .
\end{array}\right.
$$

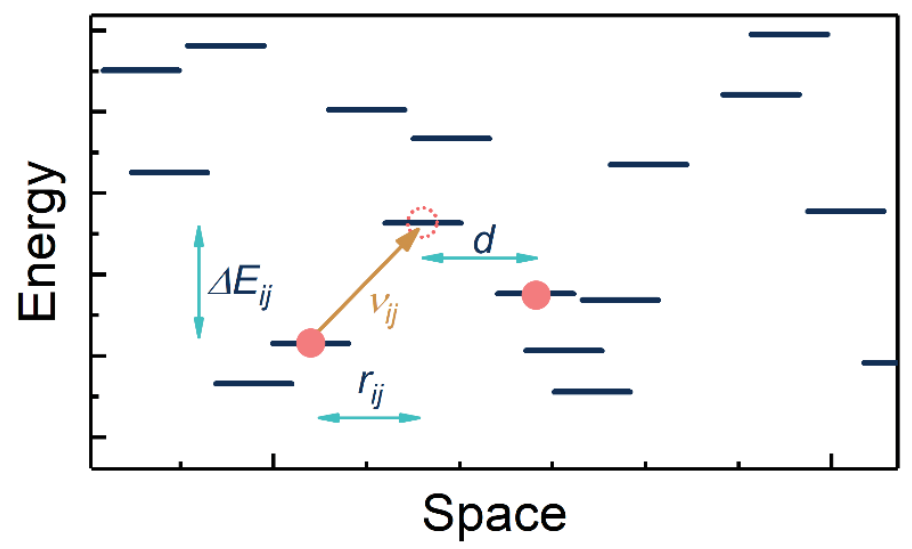

Figure 2.6 Charge carrier hop between two sites with the Miller-Abrahams hopping rate determined by the separation in energy and space.

Like the attempt-to-hop frequency, the localization length $\alpha$ is material-dependent. In the work of this thesis, and in fact throughout similar literature, $\alpha$ is assumed to be somewhere between $0.1 \mathrm{~nm}$ and $2 \mathrm{~nm}{ }^{29}$.

\subsubsection{Percolation Model}

In their original work, Miller and Abrahams calculated the current flow of charges due to charge hopping by considering percolation through a resistor network. There, every hopping site is replaced by an electrical node (junction or site energy) 
distributed randomly in space. A pair of neighbouring nodes $i$ and $j$ is connected via a resistor $R_{i j}$ that is inversely proportional to the MA hopping probability as ${ }^{30}$

$$
R_{i j}=\frac{k_{B} T}{q^{2}} \frac{1}{\vartheta_{i j}}
$$

where $\vartheta_{i j}$ is the symmetric equilibrium rate of transition between sites $i$ and $j$ in Equation (2.10). Only upwards hops are considered since these are connected to a penalty in energy and thus considered to be the rate-limiting step in hopping transport. In most percolation models, the electric field is implicitly assumed to be small and only a perturbation to the resistances ${ }^{31}$. The effect of a non-vanishing field is discussed in section 2.3.

From percolation theory it follows that there exists a critical value $R_{\text {crit }}$ for the resistance that is representative for the whole system and ultimately needs to be overcome to form an continuous percolation pathway through the sample (infinite percolation cluster) and hence from $R_{\text {crit }}$ the conductivity can then be calculated. In order for a current to flow in the resistor network there needs to be a continuous path from one electrode to the other. To find $R_{\text {crit }}$ one sequentially removes resistors from the network, starting with the largest resistances. $R_{\text {crit }}$ is then the value of the resistance that is removed just before percolation stops.

Alternatively, the percolation problem can be reduced to a purely geometrical one shown in Figure 2.7 by considering $d$-dimensional spheres around every site with radii $r_{i}$ instead of pairs of sites separated by a resistance $R_{i j}$. The threshold for percolation is then found by increasing $r$ until an infinite cluster is formed at $r=r_{C}$, i.e. a continuous path from one end of the sample to the other.

For a constant DOS one can calculate the average number of sites within a sphere at the percolation limit as

$$
\frac{4 \pi}{3} g\left(E_{F}\right) r_{C}^{3}=B_{C},
$$

where $B_{C}$ is the average number of bonds per site necessary for the formation of an infinite cluster, typically referred to as percolation threshold, and $g\left(E_{F}\right)$ is the DOS at the Fermi level. Restricting the hops to only nearest neighbour hops (NNH), such as depicted in Figure 2.8, in a cubic lattice leads to $B_{C}=2.8^{32}$. 


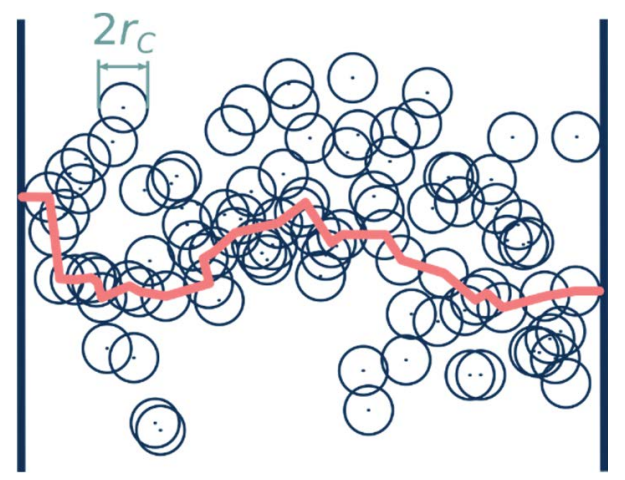

Figure 2.7 Percolation criterium interpreted with spheres.

However, since hopping is a thermally activated process, at low thermal energies $k_{B} T$, compared to the characteristic energy of the DOS, it becomes energetically favourable for carriers to tunnel to non-nearest sites with lower site energies, as shown schematically in Figure 2.8.

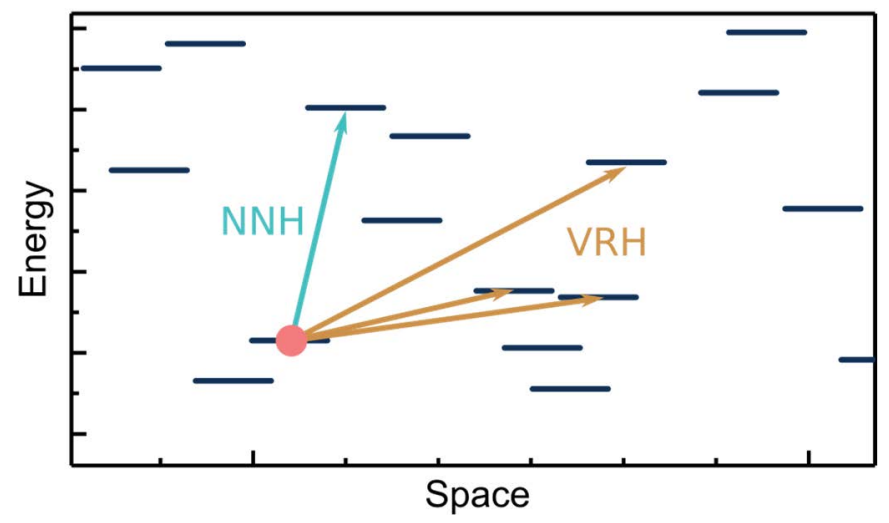

Figure 2.8 Nearest neighbour hopping (green) and variable range hopping (brown) in energy and real space.

This hopping regime is referred to as variable range hopping (VRH) and can be implemented into the percolation formalism by assuming percolation in 4 dimensions (3D space $+1 \mathrm{D}$ energy). Analogously, the percolation criterion Equation (2.15) for VRH can be written as

$$
\frac{4 \pi}{3} r_{C}^{3} \int_{E_{i}}^{E_{f}} g\left(E^{\prime}\right) d E^{\prime}=B_{C}
$$


Assuming charge transport only around the Fermi energy as well as a constant DOS around $E_{F}$, solving the percolation problem with VRH leads to the well-known Mottlaw for the conductivity at low temperatures ${ }^{33}$

$$
\sigma=\sigma_{0} \exp \left(-\left(\frac{T_{0}}{T}\right)^{\frac{1}{1+d}}\right) .
$$

The prefactor $\sigma_{0}$ depends only weakly on temperature. The characteristic temperature $T_{0}$ is defined as

$$
T_{0}=\frac{\beta \alpha^{d}}{k_{B} g_{0}}
$$

with $\beta$ a numerical constant. As mentioned earlier, the DOS of most ODSC is believed to be of exponential or Gaussian shape. The Mott-law Equation (2.17) is only valid in the approximation of a constant DOS around $E_{F}$ which for strongly energy-dependent DOS distribution can only be considered to be the case at low temperatures (the critical temperature for Mott-like VRH is dependent on the disorder and shape of the DOS see e.g. Ref ${ }^{7}$ ). Additionally, it is not straightforward to assume that the numerical value of $B_{C}=2.8$ found for a constant DOS and NNH in a cubic lattice can be generalized to VRH between randomly distributed sites and other DOS shapes ${ }^{7}$. The average number of bonds necessary for VRH has thus far only been solved explicitly for an exponential DOS by considering the geometric problem of randomly distributed spheres with exponentially distributed radii ${ }^{34}$. Other authors usually assume $B_{C}=2.8$, which has been shown to only lead to small deviations of the calculated mobilities ${ }^{34}$.

\subsubsection{Application to Exponential and Gaussian Density of States}

Common to all percolation models with an exponential DOS is that the charge carrier mobility $\mu$ is activated by the transfer between $E_{F}$ and a critical transport energy $E_{\text {perc }}^{*}{ }^{7,35}$

$$
\mu \propto \exp \left(-\frac{E_{\text {perc }}^{*}-E_{F}}{k_{B} T}\right)=\exp \left(-\frac{\Delta E}{k_{B} T}\right)
$$

where $E_{\text {perc }}^{*}$ does not depend on $E_{F}$ and is thus independent on the charge carrier concentration. The physical meaning of $E_{\text {perc }}^{*}$ can be interpreted as the most difficult 20 
hop a carrier has to make in order for it to be transported through the sample. However, $E_{\text {perc }}^{*}$ is not the energy at which transport is most likely to happen, this is only the case for $\mathrm{NNH}$.

Percolation with VRH in a Gaussian DOS has been solved explicitly only for a lattice model and small concentrations. The model describing this transport is usually referred to as the Gaussian disorder model (GDM) ${ }^{36}$. The mobility for a Gaussian DOS has a stronger temperature dependence than the exponential VRH regime, namely $\mu \propto \exp \left(-\Delta E / k_{B} T^{2}\right)^{6}$. However, in the limit of low concentrations, VRH in exponential DOS and the GDM lead to fundamentally different concentration dependences of the mobility due to the carriers sitting at the equilibrium energy $E_{\infty}=-\sigma^{2} / k_{B} T$, rather than the Fermi level ${ }^{3}$.

At very large disorders, the DOS around the Fermi level can be assumed constant and Mott-type VRH is recovered. At large temperatures (compared to the other energy scales of the system) and large concentrations it is usually assumed that NNH is the dominant charge transport, since thermal activation allows the carriers to find a suitable nearest neighbour for percolation.

\subsubsection{Application to Arbitrary DOS shape}

From Equation (2.11) and Equation (2.14) it follows that the conductivity can be written as

$$
\sigma=\sigma_{0} \exp \left(-\frac{2 R^{*}}{\alpha}-\frac{E^{*}-E_{F}}{k_{B} T}\right)
$$

where $R^{*}$ is the hopping distance in space and $E^{*}$ is the energy to which carriers hop from the Fermi level ${ }^{37,38}$. The optimal hopping distance-energy combination depends on temperature, localization length and DOS, $g(E)$, and can be obtained by minimizing the exponent in Equation (2.20). For VRH the hopping distance between two sites is given by the percolation criterion Equation (2.16) and follows to

$$
\mathrm{R}^{*}=\left[\frac{4 \pi}{3 B_{C}} \int_{E_{F}}^{E^{*}} g\left(E^{\prime}\right) d E^{\prime}\right]^{-\frac{1}{3}} .
$$


The transport energy $E^{*}$ is then the final site energy $f$ for which the upwards hopping rate $\vartheta_{\text {up }}$ from an initial site $i$ is highest by solving

$$
\frac{\partial \ln \left(\sigma\left(\mathrm{R}^{*}, \varepsilon_{\mathrm{i}}, \varepsilon_{\mathrm{f}}\right)\right)}{\partial E_{f}}=0 .
$$

The transport energy (TE), schematically depicted in Figure 2.9, is, in good approximation, independent on the initial energy (Fermi level) and thus independent on the carrier concentration. The position is only dependent on the shape and width of the DOS. It has been shown, in fact, that for a Gaussian DOS, $E^{*}$ sits at a constant offset from the peak of the DOS ${ }^{35}$. The mobility and conductivity in the TE model are activated and have thus the same functional dependence as Equation (2.19) with $E^{*}$ instead of $E_{\text {perc }}^{*}$. In fact, it has been shown that the transport energy is valid for every steeply energy-dependent DOS ${ }^{39}$.

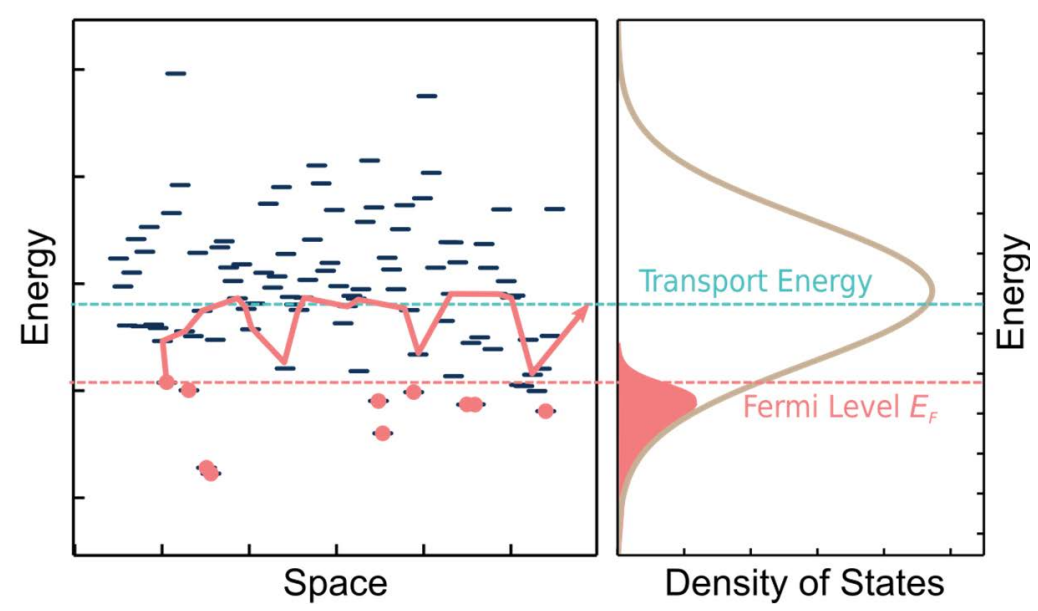

Figure 2.9 Schematic representation of the transport energy $E^{*}$ around which most transport occurs.

Throughout this thesis both the VRH and NNH concepts were used with different justifications. Due to computational restraints, numerical investigations were only done in the NNH regime. However, justifications and the correctness of the results have been given throughout the scientific work of this thesis. 


\subsubsection{Our Contribution}

Distinguishing between VRH and NNH from concentration and temperature dependence of the conductivity is difficult, since many models give the same qualitative dependence on temperature and carrier concentration for both types of hopping. Additional insight into the transport mechanism can be gained by a thermoelectric investigation as it gives direct insight into the energetics (described in section 3). The occurrence of a quasi-universal conductivity-thermopower relationship for many doped systems and spanning several orders of magnitude in conductivity points towards a common transport mechanism in a wide variety of materials. However, none of the typically employed models is able to describe such a relation. In Paper III we show that VRH transport in a DOS that is broadened and exponentially extended by the presence of ionized dopants, leads to the experimentally observed power-law relationship. This further indicates that in most doped systems, significant amount of hopping beyond nearest neighbours occurs at room temperature

\subsubsection{DOS Modification due to Presence of lonized Dopants}

Doping is one way of increasing the density of free charge carriers, where the dopant transfers charges to the host. What remains is an ionized dopant that sits within the randomly distributed sites of the host material. As mentioned in section 2.1, the presence of a charged species has an influence on the shape and width of the DOS. The ionized dopant interacts Coulombically with sites in the vicinity and therefore their presence modifies the DOS of the host-guest system and the energetic landscape in which the charge carriers perform their hops and thus charge transport.

Arkhipov et al. were the first to include this effect into an analytical theory, of percolative charge transport by VRH in an initially Gaussian DOS ${ }^{9,40}$. If the number of dopants $N_{D}$ is small compared to the number of intrinsic sites $N_{i}$, the ionized dopants will only interact with the intrinsic localized sites and not with each other $\left(N_{D}^{-3}<\right.$ $\left.r_{C}\right)$. The probability of finding an ionized dopant at a distance $a$ from a localized site is given by the Poisson distribution and the initially Gaussian sites energy distribution $E_{\text {site }}$ is modified by the distribution of Coulomb energy $E_{C}$ due to the dopant ions. The new total site energy is then $E=E_{C}+E_{\text {site }}$. The effect on the DOS is a shift of site energies within $r_{C}$ of the ions to lower energies which extends the tail exponentially ${ }^{41}$. 
Common hopping transport models only take into account an intrinsic DOS (i.e. Equation (2.2) or (2.3)). Since the relative dielectric constant of ODSC typically ranges between 2-4, which is relatively small compared to inorganic materials with $\varepsilon_{r}$ around 11 , the Coulomb capture radius $r_{C}=10-20 \mathrm{~nm}$ and thus much larger than the average distance between hopping sites $a_{N N}=0.8-2 \mathrm{~nm}$ (see section 2.2.1). Consequently, a single dopant ion effects several hundred hopping sites, which should have a strong influence on charge transport.

To facilitate analytical treatment within the VRH formalism Arkhipov et al. employed two simplifications to the physical model;

i) the Coulomb traps within $r_{C}$ of a dopant ion are replaced with a single trap site and ii) the energy of these sites is the sum of the intrinsic, disorder-dependent site energy and the barrier between these sites modified by the presence of an external electric field $F, E_{\text {trap }}=E_{\text {site }}+\Delta_{\text {ion }}$, with

$$
\Delta_{i o n}=\sqrt{\frac{q^{3} F}{\pi \varepsilon_{0} \varepsilon_{r}}}-\frac{q^{2}}{4 \pi \varepsilon_{0} \varepsilon_{r} a},
$$

where $a$ is the distance between a dopant ion and the nearest hopping site. The barrier $\Delta_{i o n}$ is schematically indicated on the left side of Figure 2.10. The modified DOS takes the form

$$
g(E)=\frac{N_{i}-N_{D}}{N_{i}} g_{i}(E)+\frac{N_{D}}{N_{i}} g_{i}\left(E-\Delta_{i o n}\right),
$$

where $g_{i}$ is the intrinsic DOS. Transport is then calculated from a percolative transport energy model assuming VRH but can in principle be applied to any other hopping model that takes the DOS into account. The calculated mobility follows the Poole-Frenkel behaviour in field, $\mu \propto \sqrt{F}$, while the temperature dependence follows Arrhenius behaviour. Interestingly, the concentration dependence of the mobility is very sensitive on the initial disorder of the DOS. For small disorders, the mobility exhibits a negative concentration dependence while an increasing mobility is found for very large disorders. This can be related to the position of the majority of charge carriers in energy, i.e. the equilibrium energy $E_{\infty}$, which scales with disorder and surpasses the value for activation from a Coulomb trap at a disorder of $0.15 \mathrm{meV}$. At this point charge carriers fill the traps and the Fermi energy increases with dopant concentration and thereby also the mobility. However, the conductivity increases also 
for small disorders, since the mobility decreases at a slower rate than the concentration of free charge carriers, $N_{D}$, increases.

At dopant concentrations above approximately $0.1 \%$ the distance between dopant ions is smaller than their Coulomb capture radius $r_{C}$ leading to the overlap of the Coulomb wells of the ionized dopants seen on the right side of Figure 2.10. This is beneficial for charge transport since it smoothens the energy landscape and reduces the barrier $\Delta_{i o n}$ for de-trapping compared to the case of low concentrations without any significant overlap depicted on the left side of Figure 2.10.
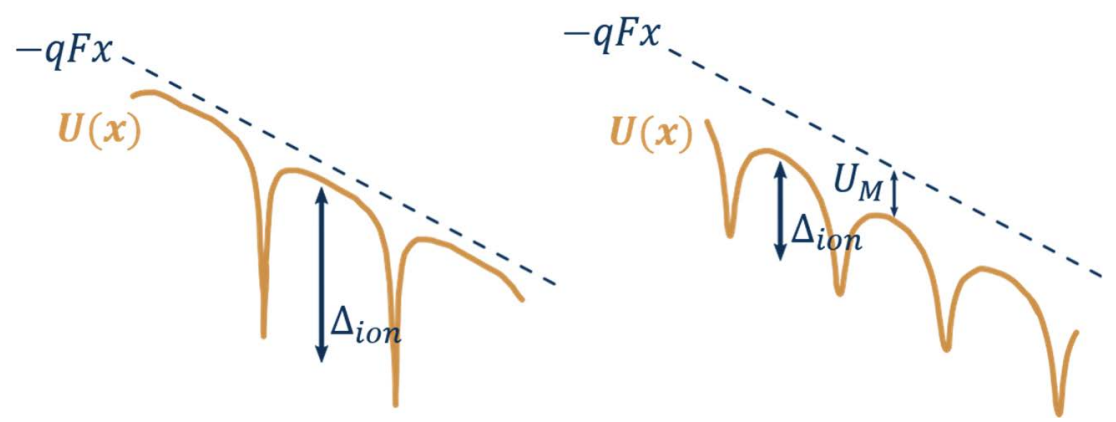

Figure 2.10 (left) Negligible overlap of dopant ion Coulomb potentials $U(x)$ at low concentrations. (right) High concentration of dopant ions increases the overlap and reduces the barrier $\Delta_{i o n}$. The energy at maximum overlap is indicated as $U_{M}$.

The energy barrier is then expressed as

$$
\Delta_{i o n}=\frac{q^{2}}{4 \pi \varepsilon_{0} \varepsilon_{r} a}+U_{M}
$$

where $U_{M}$ is the energy at maximum overlap. This addition explains the experimentally observed steep increase of the mobility at higher dopant concentrations of e.g. $\mathrm{F}_{4} \mathrm{TCNQ}$ doped $\mathrm{P} 3 \mathrm{HT}^{42}$. The effect on the DOS is paramount to a smaller copy of the DOS that is shifted towards lower energies. The dopant DOS increases and moves towards the main DOS with increasing dopant concentration, however, no change to the intrinsic peak occurs. 


\subsubsection{Our Contribution}

In Paper II we extend the expression for the DOS including the distribution of dopant ions obtained by Arkhipov et al. ${ }^{9}$ to include the energy offset between the characteristic energies of host and guest material (i.e. the HOMO of the host LUMO of the dopant material). The effect of increasing the carrier concentration on the DOS with this inclusion is then a simultaneous broadening of the main peak and formation of an exponential tail of the otherwise Gaussian DOS, depicted in Figure 2.11 for different dopant concentrations.

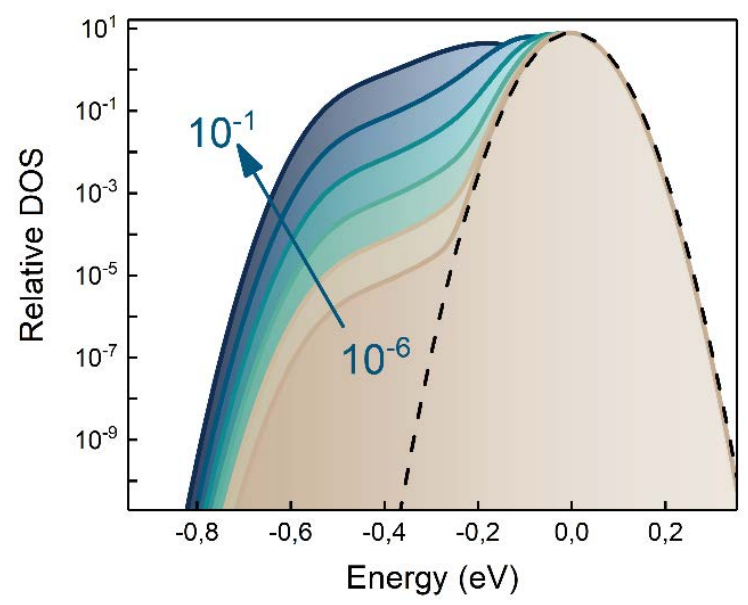

Figure 2.11 Modification of the DOS due to the presence of dopant ions ranging in relative concentration from $10^{-6}$ to $10^{-1}$ calculated with the model described in Papers II and III. The dashed line indicates the intrinsic DOS without dopants.

The transport in this model is calculated by the Mott-Martens model described in section 2.2.4.2, by activation from the Fermi energy to a transport energy ${ }^{37,38}$. The addition to the modification of the DOS allows for a more precise description of experiments as shown in Paper II. In Paper III the Arkhipov DOS modification is used to distinguish between $\mathrm{VRH}$ and $\mathrm{NNH}$ regime within the context of the conductivity dependence of the thermopower (further details can be found in section 3.3.6). Additionally, we find that the model based on the DOS described by Equations (2.25) and (2.24) does neither reproduce the DOS modification nor the concentrations dependence of the mobility calculated with MC simulations. 


\subsubsection{Short Range Carrier-Carrier interaction and Coulomb Gap}

At larger charge carrier concentrations, however, one would expect the inter-carrier Coulomb interactions to play a (significant) role in charge transport, due to the changes they infer on the DOS (section 2.1) and modification of the MA hopping rate (Equation 2.11). Pollak and Ambegaokar have in fact shown that the short-range interaction between localized charge carriers leads to a decrease of the DOS around the Fermi energy ${ }^{30,43}$. This has strong implications on the charge transport (especially at low temperatures) since the Mott-law, which is often used as a reference model to classify hopping transport, requires the DOS at the Fermi level to be constant. Efros and Shklovskii have developed a model describing the shape of the DOS around $E_{F}$ in a system of interacting charges and were amongst the first to study the influence of the Coulomb gap on conductivity ${ }^{44}$. Assuming negligible wavefunction overlap (i.e. assuming localization of states) the energy of a system must increase upon transferring a carrier from a site below the Fermi energy to a site above the Fermi energy, as depicted in Figure $2.12^{44}$.

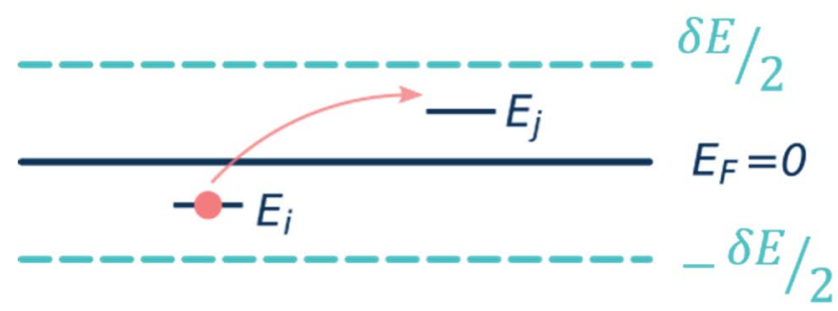

Figure 2.12 Transfer of charge carrier from an occupied site below the Fermi level to an unoccupied site above the Fermi level $E_{F}$.

Thus, the inequality

$$
\Delta_{i j}=E_{j}-E_{i}-\frac{q^{2}}{4 \pi \varepsilon_{0} \varepsilon_{r} r_{i j}}>0
$$

must be fulfilled for every possible hop from below $E_{F}$ to above $E_{F}$. The last term in the middle part of Equation (2.22) takes into account the reduction of the hopping energy by the attractive potential between the hopping charge carrier type (electron or hole) and the vacancy it leaves behind (hole or electron). Imagining a small energy interval $\delta E$, indicated by the dashed lines in Figure 2.12, around the Fermi level and assuming sites to be randomly distributed in space with an average concentration of 
$N_{\text {site }}$, one can assume the average spatial separation $\xi$ between these sites to be (per Equation (2.1))

$$
\xi=\left(\frac{1}{g\left(E_{F}\right) \delta E}\right)^{\frac{1}{3}},
$$

where $g\left(E_{F}\right)$ is the DOS at the Fermi level. In order for inequality (2.26) to be satisfied, sites must be separated by a distance of at least

$$
\xi \geq \frac{q^{2}}{4 \pi \varepsilon_{0} \varepsilon_{r} \delta E}=\xi_{C} .
$$

This also puts a constraint on the site density $N$ around $E_{F}$, which in three dimensions may not be larger than $\xi_{C}^{-3}$ or

$$
N_{C}=\frac{\delta E^{3}\left(4 \pi \varepsilon_{0} \varepsilon_{r}\right)^{3}}{q^{6}} .
$$

This constriction leads to a decreasing DOS around $E_{F}$ and ultimately to the formation of a soft Coulomb gap, depicted schematically in Figure 2.13.

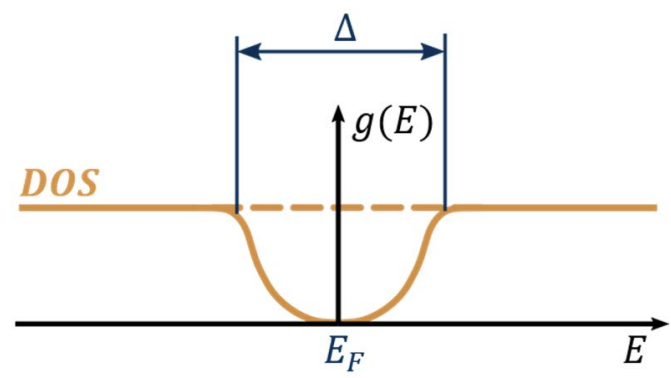

Figure 2.13 Efros-Shklovskii Coulomb gap with width $\Delta$ forming around the Fermi level $E_{F}$ of an otherwise constant DOS.

The width $\Delta$ of the gap follows from equating the unperturbed DOS $g_{0}$ with the gap DOS, $g(E)$, which in 3D follows to

$$
g(E)=\frac{d N_{C}}{d E}=C \frac{\delta E^{2}\left(4 \pi \varepsilon_{0} \varepsilon_{r}\right)^{2}}{q^{6}}
$$

with the numerical constant $C=3 / \pi$ and the gap width 


$$
\Delta=\frac{q^{3} \sqrt{g_{0}}}{\left(4 \pi \varepsilon_{0} \varepsilon_{r}\right)^{\frac{3}{2}}} .
$$

Due to the inherent spread of site energies (i.e. disorder), the Coulomb gap for most ODSCs does not open completely at $E_{F}$.

The effect of the Coulomb gap on transport is only important if the gap width is of the same order as $k_{B} T$, then the presence of the gap leads to a conductivity dependence in two and three dimensions that is similar to the Mott-law in one dimension, however, with different prefactor and constants,

$$
\sigma=\sigma_{E S} \exp \left(-\left(\frac{T_{E S}}{T}\right)^{\frac{1}{2}}\right)
$$

where $T_{E S}=\beta_{E S} q^{2} / 4 \pi \varepsilon_{0} \varepsilon_{r} \alpha$, with $\beta_{E S}$ a numerical coefficient. 


\subsection{NON-OHMIC CHARGE TRANSPORT}

At field energies that are large compared to the available thermal energy, i.e. $q F x / k_{B} T \gg 1$, the often referred to Mott-law is not valid anymore. It is quite clear, however, that study and description of the high field and high temperature regimes are of importance not only for applications but also for gaining a deeper understanding of the charge transport mechanisms. To that end, Pollak \& Riess extended the original percolation formalism to higher fields leading to a temperature and field-dependent expression of the conductivity for VRH in a constant DOS that is valid at intermediate fields ${ }^{31}$

$$
\sigma(F, T)=\sigma_{0} \exp \left(c \frac{q F L(T)}{k_{B} T}\right),
$$

where $\sigma_{0}$ is the (temperature dependent) ohmic conductivity, $c$ is a constant related to the angle between electric field and hopping direction (typically chosen to be 0.17 ${ }^{45,46}$ ) and $L(T)$ is the length scale characteristic for the hopping process. The precise connection of $L(T)$ to the material is dependent on the specific morphology and should not be taken too literal. However, study of this length scale can give interesting insights into charge transport and indicate changing hopping behaviour.

\subsubsection{The Reduced Activation Energy}

At low temperatures ODSCs with an approximately constant DOS at the Fermi level exhibit a Mott-like temperature dependence of the conductivity

$$
\sigma \propto \exp \left(-\left(\frac{T_{0}}{T}\right)^{\gamma}\right)
$$

where the exponent $\gamma=1 /(1+d)$ ranges between $1 / 2-1 / 4$ depending on the number of dimensions in which charge transport occurs and thus indicating VRH in 1 to 3 dimensions (section 2.2.4). The exponent, and thereby the dimension of this type of charge transport, is often determined by the temperature dependence of the socalled reduced activation energy ${ }^{47}$

$$
W(T)=\frac{d \ln (\sigma)}{d \ln (T)}
$$


An early study of the ohmic conductivity of doped polyaniline showed a metalinsulator transition at sufficiently low temperatures ${ }^{48}$. By application of a magnetic field, an insulating behaviour with a Mott-VRH temperature dependence was created, while reducing the energetic disorder created metallic conduction. The "untreated" films, however, fall into a critical regime, characterized by a power-law temperature dependence, $\sigma \propto T^{\alpha}$, with the exponent $\alpha$ typically between 0.26 and $0.4{ }^{62}$.

Mott-VRH in 3D leads to a slope of $-1 / 4$, the metallic state has a positive slope and the reduced activation energy of the critical regime is constant in temperature. A transition from semiconducting to critical behaviour has also been observed for PEDOT doped with PSS upon treatment with different solvents ${ }^{49}$. The temperature dependence of the reduced activation energy of DMSO treated PEDOT:PSS is in the critical regime, while pristine, DMF and THF-treated PEDOT:PSS exhibits semiconducting behaviour with different slopes, i.e. different dimensionalities according to the Mott model. The pristine sample exhibits a slope of $-1 / 2$ indicating one-dimensional VRH charge transport. This was subsequently confirmed by further investigations of the reduced activation energy, which attributed this behaviour to quasi-1D charge transport in PEDOT:PSS in parallel, weakly interacting onedimensional fibres ${ }^{45,46}$. A metallic temperature dependence was found for PEDOT:Tos and was attributed to a semi-metallic state ${ }^{50}$.

For activated transport, $\sigma \propto \exp \left(-\Delta E / k_{B} T\right)$, the reduced activation energy takes the form,

$$
W(T)=\frac{\Delta E}{k_{B} T} \frac{d \Delta E}{d T} \propto \frac{1}{T^{\alpha a c t}},
$$

where $\alpha_{a c t}$ depends on the choice of the energy from which most carriers are activated from. In the GDM (i.e. Boltzmann limit and Gaussian DOS) $\Delta E=E^{*}-E_{\infty}$, with $E_{\infty}=-\sigma^{2} / k_{B} T$ introducing an additional temperature dependence leading to $\alpha_{\text {act }}=2$, while $\Delta E=E^{*}-E_{F}$ leads to $\alpha_{\text {act }}=1$.

\subsubsection{Universal Scaling}

In the Ohmic regime, highly conducting systems close to the metal-insulator transition often exhibit a power-law dependence of the current on the temperature, rather than the stretched exponential behaviour expected from hopping transport;

$$
j \propto T^{\alpha} \quad\left(k_{B} T>>q V\right),
$$


while for the non-ohmic regime (at large voltages) a power-law dependence on voltage $V$ is observed

$$
j \propto V^{\beta} \quad\left(k_{B} T \ll q V\right),
$$

where the exponents $\alpha$ and $\beta$ are often found to be related as

$$
\beta=\alpha+1 .
$$

The combined influence of field and temperature has been shown to collapse to a universal line that transitions from ohmic to non-ohmic behaviour when plotted as $J / T^{1+\alpha}$ vs. $V / T$ and can be described by the expression ${ }^{51-56}$

$$
j=b T^{1+\alpha} \sinh \left(\gamma \frac{q V}{k_{B} T}\right)\left|\Gamma\left(\frac{1+\beta}{2}+\frac{i}{\pi} \gamma \frac{e V}{k_{B} T}\right)\right|^{2},
$$

where $b$ is a scaling parameter, $\gamma$ a parameter describing the transition between ohmic and non-ohmic regime such that $2 / \gamma \approx q V / K_{B} T$ and $\Gamma$ is the complex Gamma function. An example of the collapse to a universal line of experimental data obtained from PEDOT:PSS is seen in Figure 2.14. From conductivity measurements on the left side of Figure 2.14 the temperature dependence of the ohmic conductivity (i.e. field independent) the exponent $\alpha$ is obtained and the data of the complete field range plotted in rescaled units on the right side of Figure 2.14.
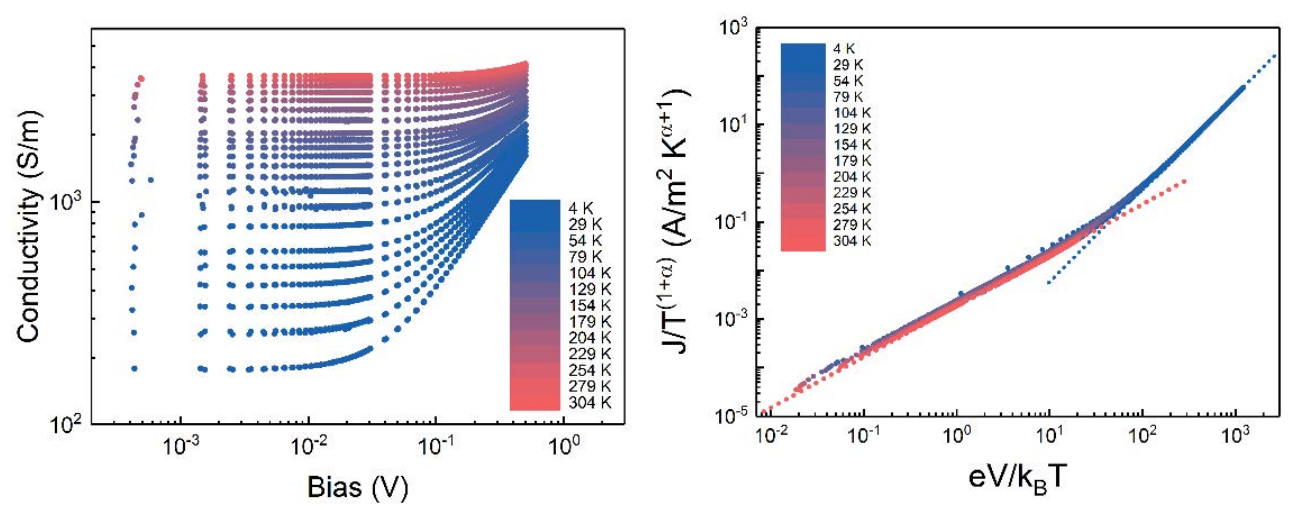

Figure 2.14 (left) Conductivity vs. applied bias for a 1:2.5 (w/w) PEDOT:PSS thin film, measured at different temperatures indicated by the color scale. (right) Same data as in (left), rescaled according to the universal scaling procedure discussed in the text. The red and blue dotted lines on the right side indicate power laws with slope 1 and $b$, respectively; $\alpha=1$. Reproduced with permission from Paper I. 
The universal scaling of temperature and field dependence has been observed for various inherently one-dimensional systems (e.g. nanotubes) as well as amorphous polymeric semiconductors such as PBTTT (as active layers in OFETs as well as doped films) or PEDOT:PSS and is mostly attributed to be a characteristic of transport in a Luttinger liquid. However, the large variety of materials in which this behaviour is observed has sparked discussions about the universal validity of the LL theory, leading to at least five mutually exclusive models leading to Equation 2.40 or qualitatively equivalent expressions as briefly discussed in Paper I

\subsubsection{Effective Temperature}

An electric field $F$, as was argued by Marianer \& Shklovskii, has a similar effect on charge hopping as the lattice temperature $T^{57}$, allowing similarities to be drawn to thermal activation of hopping transport. To a charge carrier, the energetic landscape is shifted by an energy $\Delta E_{\text {Field }}=q F x$ to lower energies (w.r.t. what is considered lower for a hole or an electron) when hopping a distance $x$ with (against) the electric field. This situation is schematically depicted on the left side of Figure 2.15 with the example of electrons in an exponential DOS.
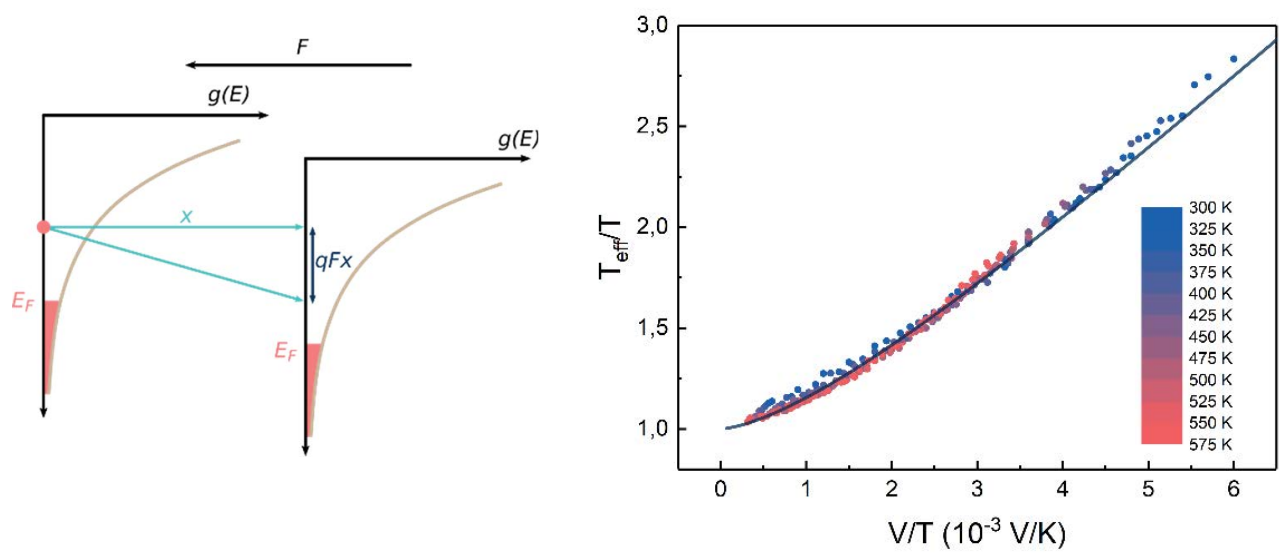

Figure 2.15 (left) Electron hop against the electric field F. Due to positive energy dependence of the DOS a higher concentration of localized sites is available for the electron. The shaded area represents the occupied states. (right) Scaled dependence of $T_{\text {eff }}$ on the field $F$, reproduced with permission from reference Paper $I$. 
Hence, hopping under the influence of a field and temperature is paramount to hopping without a field, but activated by an effective temperature $T_{\text {eff }}$ that includes the combined effect of field and temperature. The validity of $T_{\text {eff }}$ at finite temperatures has subsequently been shown by comparing measurements on amorphous Silicon to a numerical study assuming an exponential DOS and the Boltzmann limit of low concentrations ${ }^{57}$.

Numerically, $T_{e f f}$ is obtained by fitting the Boltzmann or Fermi-Dirac distribution function to the numerically obtained charge carrier distribution. This process is schematically shown in Figure 2.16. Plotting these $T_{\text {eff }}$ values as a function of the ratio between field and temperature yields a universal line as seen on the right side of Figure 2.15 that is described by the empirically expression

$$
T_{e f f}^{\zeta}(T, F)=T^{\zeta}+\left(\frac{\lambda q F \alpha}{k_{B}}\right)^{\zeta},
$$

which describes the combined effect of lattice temperature $T$ and electric field $F$ on the charge carrier distribution. The exponent $\zeta$ was originally found to be 2 , however, no physical interpretation was given ${ }^{57}$.
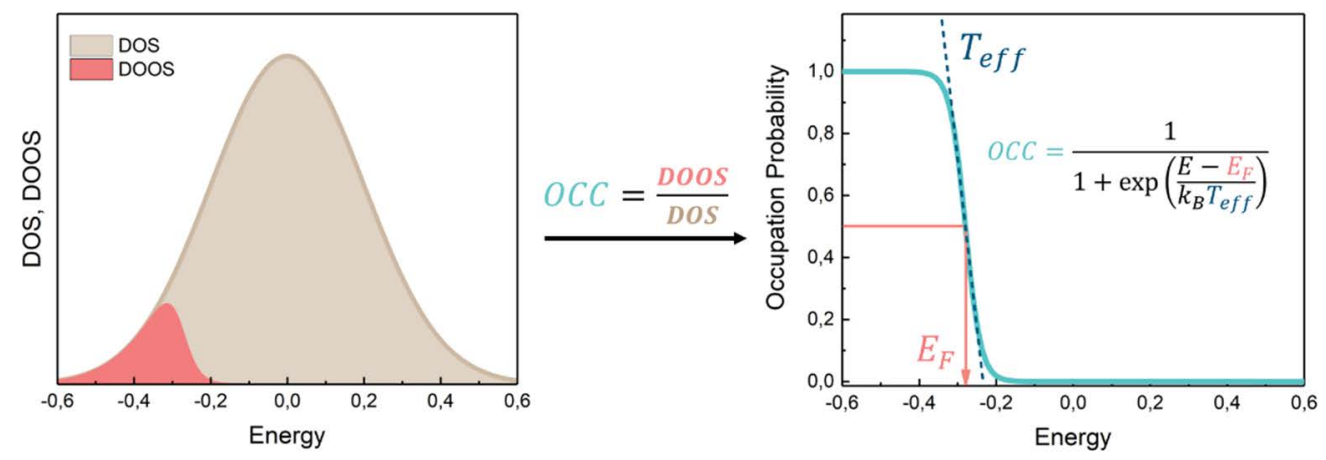

Figure 2.16 Numerical determination of $T_{\text {eff }}$ by division of the numerically obtained DOOS by the DOS and fitting of the resulting occupation probability to the Fermi-Dirac distribution.

Experimentally, $T_{\text {eff }}$ is obtained by comparing the temperature and field dependent conductivity $\sigma(T, F)$ to the field independent ohmic conductivity $\sigma(T, 0)$ and finding the temperature at which the latter is equal to the former. 
Interestingly, if the conductivity is plotted as a function of $T_{\text {eff }}$ a collapse to one line is obtained following the relation

$$
\sigma(F, T) \propto T_{e f f}^{\alpha} .
$$

The concept of describing temperature and field dependence combined via $T_{e f f}$ has subsequently been shown to be valid experimentally and numerically also for Gaussian disorders and Fermi-Dirac statistics as well as beyond the Boltzmann limit ${ }^{58-}$ ${ }^{61}$. The scaling of the conductivity to a universal curve is rather reminiscent of the previously described universal scaling, not least if one considers the equivalent axes units in both types of scaling on the right side of Figure 2.14 and 2.15.

\subsubsection{Our Contribution}

In Paper I, we show that the universal scaling and $T_{\text {eff }}$ frameworks describe the same functional dependence of the conductivity in $F$ and $T$ in Ohmic and non-Ohmic limit. Both scaling phenomena are observed for PEDOT:PSS out-of-plane via devices and kinetic Monte Carlo simulations. From physical insight obtained via numerical investigations, we find that the physical background of both types of scaling can be related to a simple heat balance between Joule heating of the current and temperature dependent relaxation to the lattice. Finally, by solving the heat balance equation, we develop an empirical model that consistently describes both scaling phenomena. 


\subsection{BIBLIOGRAPHY}

1. Brédas, J. L. Relationship between band gap and bond length alternation in organic conjugated polymers. J. Chem. Phys. 82, 3808-3811 (1985).

2. Venkateshvaran, D. et al. Approaching disorder-free transport in high-mobility conjugated polymers. Nature 515, 384-388 (2014).

3. Baranovskii, S. D. Theoretical description of charge transport in disordered organic semiconductors. Phys. Status Solidi 251, 487-525 (2014).

4. Grünewald, M., Thomas, P. \& Würtz, D. Simple calculation of the hopping conductivity for an exponential band tail. Phys. Status Solidi 94, K1-K4 (1979).

5. Vissenberg, M. C. J. M. \& Matters, M. Theory of the field-effect mobility in amorphous organic transistors. Phys. Rev. B 57, 13 (1998).

6. Bassler, H. Charge Transport in Disordered Organic Photoconductors. Phys. Status. Solidi. B, Basic Res. 175, (1993).

7. Baranovskii, S. D. Theoretical description of charge transport in disordered organic semiconductors. Phys. Status Solidi Basic Res. 251, 487-525 (2014).

8. Tanase, C., Meijer, E. J., Blom, P. W. M. \& De Leeuw, D. M. Unification of the hole transport in polymeric field-effect transistors and light-emitting diodes. Phys. Rev. Lett. 91, 216601 (2003).

9. Arkhipov, V. I., Heremans, P., Emelianova, E. V. \& Bässler, H. Effect of doping on the density-of-states distribution and carrier hopping in disordered organic semiconductors. Phys. Rev. B 71, 45214 (2005).

10. Arkhipov, V. I., Heremans, P., Emelianova, E. V. \& Bässler, H. Effect of doping on the density-of-states distribution and carrier hopping in disordered organic semiconductors. Phys. Rev. B 71, 45214 (2005).

11. Di Pietro, R. et al. Coulomb Enhanced Charge Transport in Semicrystalline Polymer Semiconductors. Adv. Funct. Mater. 26, 8011-8022 (2016).

12. Anderson, P. W. Absence of diffusion in certain random lattices. Phys. Rev. 109, 1492-1505 (1958). 
13. Elyutin, P. V., Hickey, B., Morgan, G. J. \& Weir, G. F. Diffusion and Localisation in Random Systems. Phys. Status Solidi 124, 279-286 (1984).

14. Arkhipov, V. I. \& Rudenko, A. I. Drift and diffusion in materials with traps. Philos. Mag. Part B 45, 189-207 (1982).

15. Rudenko, A. I. \& Arkhipov, V. I. Drift and diffusion in materials with traps. Philos. Mag. Part B 45, 177-187 (1982).

16. Nenashev, A. V et al. Effect of electric field on diffusion in disordered materials. \{II\}. Two- and three-dimensional hopping transport. Phys. Rev. B 81, (2010).

17. Nenashev, A. V. et al. Effect of electric field on diffusion in disordered materials. I. One-dimensional hopping transport. Phys. Rev. B - Condens. Matter Mater. Phys. 81, (2010).

18. Street, R. A., Northrup, J. E. \& Salleo, A. Transport in polycrystalline polymer thin-film transistors. Phys. Rev. B - Condens. Matter Mater. Phys. 71, 1-13 (2005).

19. Noriega, R. et al. A general relationship between disorder, aggregation and charge transport in conjugated polymers. Nat. Mater. 12, 1038-44 (2013).

20. Salleo, A. et al. Intrinsic hole mobility and trapping in a regioregular poly(thiophene). Phys. Rev. B 70, 115311 (2004).

21. Horowitz, G., Hajlaoui, R. \& Delannoy, P. Temperature Dependence of the Field-Effect Mobility of Sexithiophene. Determination of the Density of Traps. J. Phys. III 5, 355-371 (1995).

22. Nenashev, a. V. et al. Effect of electric field on diffusion in disordered materials. II. Two- and three-dimensional hopping transport. Phys. Rev. B 81, 1-11 (2010).

23. Conwell, E. M. Impurity Band Conduction in Germanium and Silicon. Phys. Rev. 103, 51-61 (1956).

24. Pines, D. ELECTRON INTERACTION IN SOLIDS. Can. J. Phys. 34, 1379-1394 (1956). 
25. Mott, N. F. ON THE TRANSITION TO METALLIC CONDUCTION IN SEMICONDUCTORS. Can. J. Phys. 34, 1356-1368 (1956).

26. Marcus, R. a. Electron Transfer Reactions in Chemistry: Theory and Experiment (Nobel Lecture). Angew. Chemie Int. Ed. English 32, 1111-1121 (1993).

27. Miller, A. \& Abrahams, E. Impurity conduction at low concentrations. Phys. Rev. 120, 745-755 (1960).

28. Kirkpatrick, S. Percolation and Conduction. Rev. Mod. Phys. 45, 574-588 (1973).

29. Larkin, A. I. \& Khmel'nitskii. Activation conductivity in disordered systems with large localization length. Sov. Phys. JETP 56, 647-652 (1982).

30. Ambegaokar, V., Halperin, B. I. \& Langer, J. S. Hopping Conductivity in Disordered Systems. Phys. Rev. B 4, 2612-2620 (1971).

31. Pollak, M. \& Riess, I. A percolation treatment of high-field hopping transport. J. Phys. C Solid State Phys. 9, 2339-2352 (1976).

32. Lorenz, C. D. \& Ziff, R. M. Precise determination of the critical percolation threshold for the three-dimensional 'Swiss cheese' model using a growth algorithm. J. Chem. Phys. 114, 3659-3661 (2001).

33. Mott, N. F. Conduction in non-crystalline materials. Philos. Mag. 19, 835-852 (1969).

34. Nenashev, A. V. et al. Advanced percolation solution for hopping conductivity. Phys. Rev. B - Condens. Matter Mater. Phys. 87, 1-9 (2013).

35. Cottaar, J., Koster, L. J. a, Coehoorn, R. \& Bobbert, P. a. Scaling Theory for Percolative Charge Transport in Disordered Molecular Semiconductors. Phys. Rev. Lett. 107, 136601 (2011).

36. Hartenstein, B. \& Bässler, H. Transport energy for hopping in a gaussian density-of-states distribution. J. Non. Cryst. Solids 190, 112-116 (1995).

37. Martens, H. C. F. et al. Understanding the doping dependence of the conductivity of conjugated polymers: Dominant role of the increasing density of states and growing delocalization. Phys. Rev. B 67, 121203 (2003). 
38. Coehoorn, R., Pasveer, W., Bobbert, P. \& Michels, M. Charge-carrier concentration dependence of the hopping mobility in organic materials with Gaussian disorder. Phys. Rev. B 72, 155206 (2005).

39. Baranovskii, S. D., Faber, T., Hensel, F. \& Thomas, P. The applicability of the transport-energy concept to various disordered materials. J. Phys. Condens. Matter 9, 2699-2706 (1997).

40. Arkhipov, V. I., Emelianova, E. V., Heremans, P. \& Bässler, H. Analytic model of carrier mobility in doped disordered organic semiconductors. Phys. Rev. B 72, 235202 (2005).

41. Silver, M., Pautmeier, L. \& Bässler, H. On the origin of exponential band tails in amorphous semiconductors. Solid State Commun. 72, 177-180 (1989).

42. Pingel, P. \& Neher, D. Comprehensive picture of $p$-type doping of P3HT with the molecular acceptor $\mathrm{F}_{4}$ TCNQ. Phys. Rev. B 87, 115209 (2013).

43. Pollak, M. Effect of carrier-carrier interactions on some transport properties in disordered semiconductors. Discuss. Faraday Soc. 50, 13-19 (1970).

44. Efros, a L. \& Shklovskii, B. I. Coulomb gap and low temperature conductivity of disordered systems. J. Phys. C Solid State Phys. 8, L49-L51 (1975).

45. Van De Ruit, K. et al. Quasi-one dimensional in-plane conductivity in filamentary films of PEDOT:PSS. Adv. Funct. Mater. 23, 5778-5786 (2013).

46. Nardes, A. M., Kemerink, M. \& Janssen, R. A. J. Anisotropic hopping conduction in spin-coated PEDOT:PSS thin films. Phys. Rev. B - Condens. Matter Mater. Phys. 76, 1-7 (2007).

47. Zabrodskii, A. G. \& Zinov'eva, K. N. Low-temperature conductivity and metalinsulator transition in compensate n-Ge. Zh. Eksp. Teor. Fiz. 86, 727-742 (1984).

48. Menon, R., Yoon, C. O., Moses, D., Heeger, A. J. \& Cao, Y. Transport in polyaniline near the critical regime of the metal-insulator transition. Phys. Rev. B 48, 17685-17694 (1993). 
49. Kim, J. Y., Jung, J. H., Lee, D. E. \& Joo, J. Enhancement of electrical conductivity of poly(3,4-ethylenedioxythiophene)/poly(4-styrenesulfonate) by a change of solvents. Synth. Met. 126, 311-316 (2002).

50. Bubnova, O. et al. Semi-metallic polymers. Nat. Mater. 13, 190-4 (2014).

51. Aleshin, A. N., Lee, H. J., Park, Y. W. \& Akagi, K. One-Dimensional Transport in Polymer Nanofibers. Phys. Rev. Lett. 93, 196601 (2004).

52. Kronemeijer, a. J. et al. Universal Scaling in Highly Doped Conducting Polymer Films. Phys. Rev. Lett. 105, 156604 (2010).

53. Kim, K. H. et al. Apparent Power Law Scaling of Variable Range Hopping Conduction in Carbonized Polymer Nanofibers. Sci. Rep. 1-8 (2016). doi:10.1038/srep37783

54. Bockrath, M. et al. Luttinger-liquid behaviour in carbon nanotubes. Nature 397, 598 (1999).

55. Rodin, a. S. \& Fogler, M. M. Apparent power-law behavior of conductance in disordered quasi-one-dimensional systems. Phys. Rev. Lett. 105, 1-4 (2010).

56. Grabert, H. \& Weiss, U. Quantum Tunneling Rates for Asymmetric Double-Well Systems with Ohmic Dissipation. Phys. Rev. Lett. 54, 1605-1608 (1985).

57. Marianer, S. \& Shklovskii, B. I. Effective temperature of hopping electrons in a strong electric field. Phys. Rev. B 46, 13100-13103 (1992).

58. Arkhipov, V. I., Emelianova, E. V. \& Adriaenssens, G. J. Field-dependent effective temperature and variable range hopping: Application to dark dc conductivity in doped a-Si:H. J. Appl. Phys. 93, 6150-6153 (2003).

59. Baranovskii, S. D., Cleve, B., Hess, R. \& Thomas, P. Effective temperature for electrons in band tails. J. Non. Cryst. Solids 164-166, 437-440 (1993).

60. Jansson, F., Baranovskii, S. D., Gebhard, F. \& Österbacka, R. Effective temperature for hopping transport in a Gaussian density of states. Phys. Rev. $B$ 77, 195211 (2008).

61. Preezant, Y. \& Tessler, N. Carrier heating in disordered organic semiconductors. Phys. Rev. B - Condens. Matter Mater. Phys. 74, 1-5 (2006). 
62. Larkin, A. I., \& Khmel'nitskii, D. E. Activation conductivity in disordered systems with large localization length. Sov. Phys. JETP, 56(3), 647-652 (1982). 



\section{THERMOELECTRICITY}

\subsection{The Seebeck and Peltier Effect}

Thermoelectricity is the direct conversion of thermal energy to electrical energy or vice-versa via the Seebeck and Peltier effects, respectively. By keeping two ends of a slab of material at different temperatures, a temperature gradient arises that changes the distribution of charge carriers shown in Figure 3.1, where the redistribution of charge carriers from states below the Fermi level to states above $E_{F}$ increases with temperature $T$ (note that the "definition" of below and above is opposite for electrons and holes). This creates a gradient in electrochemical potential along the material, which, in open circuit condition, is counteracted by an electric field. The strength of this electric field $F$ is proportional to the temperature gradient $\Delta T$ and the Seebeck coefficient $S$.
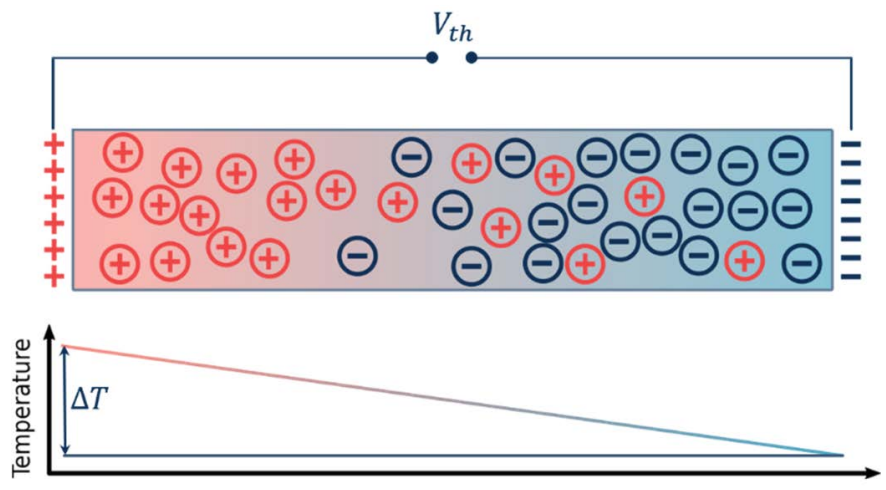

Figure 3.1 Redistribution of charge carriers due to a thermal gradient and build-up of thermal voltage under open circuit condition.

From thermodynamic considerations, the heat flux density $\tilde{q}$ and charge flux density (current density) $j$ are given by the temperature and electric potential gradients $\nabla T$ and $\nabla \varphi$, respectively, and their proportionality constants as follows

$$
\begin{gathered}
\tilde{q}=\kappa \nabla T+\Pi j \\
j=S \sigma \nabla T+\sigma \nabla \varphi,
\end{gathered}
$$


where $\kappa$ is the thermal conductivity, $\sigma$ is the electrical conductivity, $\Pi$ is the Peltier coefficient and $S$ is the Seebeck coefficient. The sign of Peltier and Seebeck coefficient depends on the type of majority carrier of the material and is positive for holes. Under the condition that there is no net charge flow (no current) and hence the potential difference, i.e. the thermal voltage $V_{t h}$ along the direction of the temperature gradient, follows to

$$
V_{t h}=S \cdot \Delta T \text {. }
$$

The Peltier coefficient, on the other hand, is the material dependent proportionality constant for the heat transported per unit time $\dot{Q}$ for a given current $I$,

$$
\dot{Q}=\Pi I \text {. }
$$

It is important to note that the transported heat is not due to Joule heating, which contrary to the Peltier effect, is an irreversible process. The Seebeck and Peltier coefficients are related to each other via the first and second Thomson relations (sometimes also referred to as Kelvin relations), which are, respectively,

$$
\mathcal{K}=\frac{d \Pi}{d T}-S
$$

and

$$
\Pi=S T,
$$

where $\mathcal{K}$ is the Thomson coefficient. From Equation (3.4) it follows that the Peltier coefficient is the amount of transported heat per electric charge. Using the second Thomson relation this makes the Seebeck coefficient the entropy transported per electric charge and hence it follows from the Boltzmann equation that

$$
S=\frac{k_{B}}{q} \ln (\Omega),
$$

where $\Omega$ is the number of equally possible configurations of the charge carriers and $k_{B} / q$ is referred to as the natural unit of the Seebeck coefficient. The total energy flux density $\dot{E}$ is the sum of the heat and energy transported by the charge carriers, which at steady state (and under the assumption of a constant current) becomes

$$
\dot{E}=\nabla \tilde{q}+j \nabla \varphi \text {. }
$$


Inserting Equations (3.1) and (3.2) into Equation (3.8) leads to

$$
\dot{E}=\nabla(\kappa \nabla T)+j \nabla \Pi+\frac{j^{2}}{\sigma}-j S \nabla T .
$$

Using the definition of the Thomson coefficient $\mathcal{K}$, Equation (3.9) can be simplified to

$$
\dot{E}=\nabla(\kappa \nabla T)+\frac{j^{2}}{\sigma}-j \mathcal{K} \nabla T,
$$

where the first term describes the heating of the material (Fourier law), the second term describes the Joule heating (resistance) and the third term the combined thermoelectric effect. 


\subsection{Applications of the Seebeck Effect}

From the energy flux density in Equation (3.10) it becomes clear that in order for a material to have a high thermoelectric efficiency, the thermal conductivity $\kappa$ must be small in order to minimize heat transport, while the electrical conductivity $\sigma$ must be large to reduce Joule heating in addition to a large Seebeck and Peltier coefficient. Since solving and comparing Equation (3.10) for different materials is rather cumbersome, comparison of the thermoelectric performance is done by means of the dimensionless figure of merit $Z T$ defined as

$$
Z T=T \frac{S^{2} \sigma}{\kappa},
$$

which increases with both increasing Seebeck coefficient and electrical conductivity and is inversely proportional to the thermal conductivity. Unfortunately, there exists and empirical inverse relationship between the Seebeck coefficient and the electrical conductivity, so that if one increases the other decreases. The thermal conductivity $\kappa$ has a lattice and an electronic contribution. The latter is connected to the electrical conductivity $\sigma$ via the Wiedemann-Franz law and cannot be greatly influenced. The former can typically be engineered to be as low as possible.

While the figure of merit is an excellent way of comparing thermoelectric performances, it is relatively hard to determine the thermal conductivity of the typically thin organic films of only several hundreds of nanometres thickness. Thus, often only the power factor

$$
P F=S^{2} \sigma
$$

is considered, with the implicit assumption that $\kappa$ does not vary significantly among organic materials. It might however become a dominating influence in composite blends, which can often also be produced in thicker films allowing for an easier thermal conductivity measurement ${ }^{1}$.

In principle, organic disordered semiconductors have a great potential for thermoelectric application due to their inherently low thermal conductivity of between $0.1-0.3 \mathrm{~W} / \mathrm{mK}$, high Seebeck coefficient and a relatively high conductivity. $Z T$ for the best performing inorganic semiconducting materials is around $1-1.2$ whereas values of up to 0.25 have been reported for organic materials (PEDOT:Tos) ${ }^{2}$. 
Most high performing inorganic materials reach their maximum $Z T$ at temperatures exceeding $100^{\circ} \mathrm{C}$, making them useful for recovering waste heat from exhaust systems in automotive and industrial applications where high temperatures are found. ODSCs, however, are not stable at temperatures significantly higher than around $80^{\circ} \mathrm{C}$, which limits their use to room temperature applications such as powering small devices from body heat, cooling from room temperature as well as for thermal sensing and temperature controlling. At the same temperature range, the inorganic semiconductor alloy $\mathrm{Bi}_{2} \mathrm{Te}_{3}$ shows the highest $Z T$ of around $1^{3}$. However, the solution processability and flexible nature of ODSCs in principle allows them to be massproduced at a very low cost thus landing a cost-advantage over inorganic materials, which often contain exotic and/or toxic materials and are difficult to produce. This explains the surge of thermoelectric investigations of organic materials ${ }^{4}$, which lead to many new material systems such as polymer-carbon nanotube (CNT) blends and nano-structuring that are believed to improve charge and energy transport, while simultaneously decreasing the lattice contribution of the thermal conductivity by phonon scattering at CNT interfaces ${ }^{1,5}$.

Apart from practical applications, the thermoelectric effect can aid charge transport investigations by giving unique insights into the energetics of the material and directly coupling it to charge transport, which is evident from Equations (3.4) and (3.7). Furthermore, thermoelectric data is relatively easy to obtain experimentally, as described in section 6 . 


\subsection{Thermoelectric TRANSPort MOdels - Conductivity AND TeMPERATURE DEPENDENCE}

\subsubsection{General Expression for the Seebeck Coefficient}

Many models describing charge transport of organic disordered semiconductors assume some type of activation from around the Fermi level $E_{F}$ to another energy $E$ (or energy band above) where transport occurs (see section 2.2.2 and 2.2.4). As mentioned before, the Peltier coefficient $\Pi$ is the amount of energy transport per charge.

The contribution of a charge carrier sitting at an energy $E$ to $\Pi$ is proportional to the conductivity contribution $\sigma(E)$ of the same carrier within the interval $d E$ to the total conductivity $\sigma^{6}$, i.e. proportional to $\sigma(E) d E / \sigma$, where

$$
\sigma(E)=q g(E) \mu(E) f(E)(1-f(E)),
$$

and the total conductivity follows to

$$
\sigma=\int \sigma(E) d E
$$

with $g(E)$ the density of states, $\mu(E)$ the mobility and $f(E)$ the distribution function, which is typically chosen to be the Boltzmann distribution function for low concentration and the Fermi-Dirac distribution function for other cases (see section 2.1). Summing up all contributions, the Peltier coefficient becomes

$$
\Pi=\frac{1}{q} \int\left(E-E_{F}\right) \frac{\sigma(E) d E}{\sigma} .
$$

With the second Thomson relation it follows for the Seebeck coefficient

$$
S=\frac{\Pi}{T}=\frac{k_{B}}{q} \int\left(\frac{E-E_{F}}{k_{B} T}\right) \frac{\sigma(E) d E}{\sigma} .
$$

In good approximation, the conductivity DOS from Equation (3.15), shown in Figure 3.2, can assumed to be symmetric and to exhibit a narrow peak at which the transport energy $E^{*}$ sits, which is then defined as

$$
E^{*}=\int \frac{E \sigma(E) d E}{\sigma} .
$$


Substituting Equation (3.17) into the expression for the Seebeck coefficient, Equation (3.16), gives,

$$
S=\frac{k_{B}}{q} \frac{E^{*}-E_{F}}{k_{B} T} .
$$

Hence, the Seebeck coefficient proportional to the difference between Fermi level and transport energy as shown in Figure 3.2. Apart from the assumption of the presence of a transport energy $E^{*}$, Equation (3.18) is universal and can lead to various different temperature and conductivity dependences of the Seebeck coefficient depending on how $E^{*}$ is calculated and what the energetic landscape looks like. An investigation of this type was undertaken in Paper III by means of the analytical model described in section 2.2.7. The kinetic Monte Carlo Algorithm used in Papers $\mathbf{V}$ and VI uses Equation (3.18) for the thermopower calculations as well but does not implicitly rely on any of the assumptions leading to Equation (3.18), since the MC algorithm allows to directly measure the mean transported energy from the positions of the charge carriers in energy.

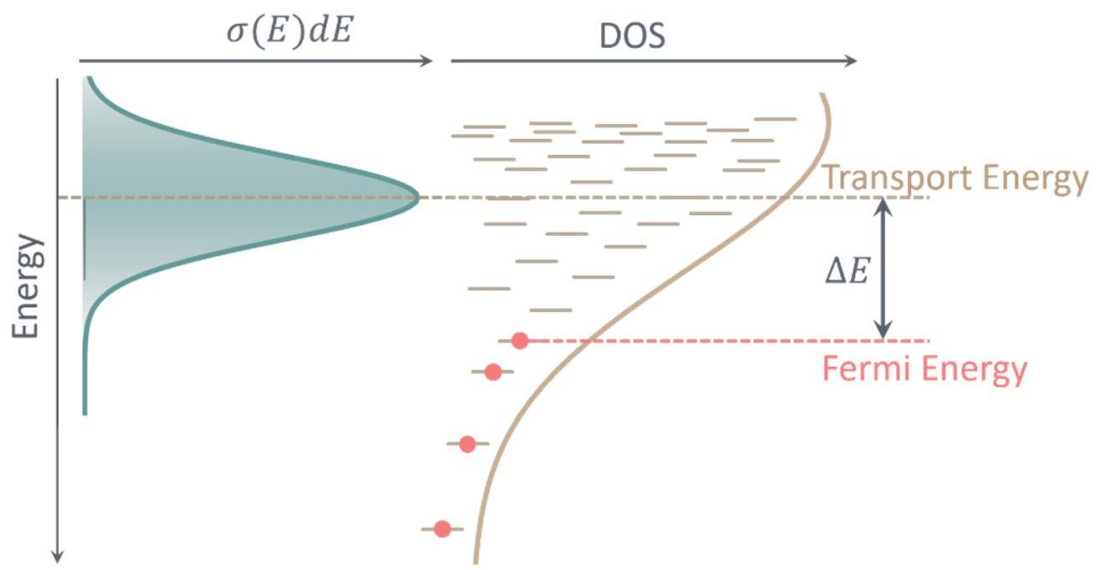

Figure 3.2 (left) Distribution of the differential conductivity. (right) Schematic depiction of the DOS with Fermi level $E_{F}$ and transport energy $E^{*}$ indicated. 


\subsubsection{Seebeck Coefficient from Statistical Mechanics}

The identification of the Seebeck coefficient as the transported entropy per charge allows to give a first order approximation of the dependence of $S$ on $\sigma$ from statistical mechanics ${ }^{7}$. Assuming a system with a relative charge carrier concentration of

$$
c=\frac{n}{N},
$$

with $n$ non-interacting charge carriers and $N$ possible sites for the carriers to occupy, the Seebeck coefficient from Equation (3.7) then shows a logarithmic dependence on concentration ${ }^{10-12}$

$$
S=\frac{k_{B}}{q} \ln \left(\frac{c}{1-c}\right),
$$

which changes sign above half-filling of the available states. Equation (3.20) is the Mott-Heike formula which is used to describe the thermopower in inorganic semiconductors ${ }^{10}$. Note that this expression of the thermopower lacks a temperature dependence. However, it might still be a good first order approximation due to the typically small linear temperature dependence of the thermopower expected in systems where charge transport is mainly due to hopping and concentrations are small (contrary to the temperature dependence of the conductivity) ${ }^{9,11,12}$. One can define a maximum conductivity $\sigma_{\max }$, from Equation (3.13) for small concentrations $c \ll 1$ by extrapolating to $c=1$ and express the conductivity with respect to $\sigma_{\max }$ leading to ${ }^{7}$

$$
\sigma_{\max }=q g \mu
$$

and

$$
\sigma=\sigma_{\max } c(1-c)
$$

or

$$
\frac{\sigma}{\sigma_{\max }}=c \quad c \ll 1 .
$$

Inserting Equation (3.23) into the expression for the thermopower Equation (3.20) results in a logarithmic dependence of the thermopower on the conductivity,

$$
S=\frac{k_{B}}{q} \frac{1}{\beta} \ln \left(\frac{\sigma}{\sigma_{\max }}\right),
$$


similar in functional dependence to the mobility edge model discussed below and plotted on the right side of Figure 3.3, where the temperature and conductivity dependence for various models is plotted together. The prefactor $\beta$ is defined by the maximum conductivities of holes and electrons as

$$
\beta=\frac{r+1}{r-1},
$$

With $r$ the ratios between the conductivities of the two types of carriers,

$$
r=\frac{\sigma_{\max }(\text { hole })}{\sigma_{\max }(\text { electron })} .
$$

Hence, if only one type of carrier is responsible for conduction, as is the case in chemical doping where the free charge carrier is introduced by a dopant molecule that remains ionized and immobile, the conductivity of the free species will be far greater than of the immobile dopant ion and consequently $r \gg 1$ and $\beta=1$ for hole conduction and $r \approx 0$ and $\beta=-1$ for electron conduction. In the case of $\beta=|1|$, Equation (3.24) transforms into the Mott-Heike formula Equation (3.20).

In Ref. ${ }^{7}$ Mateeva et al. found that Equation (3.24) fits the conductivity dependence of the thermopower measured for doped polyaniline and polypyrrole and gives $\beta \approx 9$ implying that ambipolar charge and energy transport is present in these materials. Doped polyacytelene, on the other hand, exhibits values of $\beta \approx 1$ and overall much higher Seebeck coefficients.

In Ref. ${ }^{8}$, Emin considered charge transport by hopping of bipolarons (quasi particles comprised of two bound charges in spatial vicinity and their combined effect on the structure around them). Taking into account the possibility of bipolaron splitting, the authors derived an expression similar to Equation (3.24) extended by the change in entropy caused by polaron splitting. In Ref. ${ }^{9}$ it was assumed that most carriers form bipolarons and Equation (3.24) was recovered and used to qualitatively explain the logarithmic conductivity dependence of the thermopower of $\mathrm{PF}_{6}$ - doped $\mathrm{P} 3 \mathrm{HT}$. This material exhibits a weak $T^{-1}$ temperature dependence, where the slope was found to decrease with doping concentrations. While Equation (3.24) does not explicitly contain a temperature dependence, the experimental results can be fitted by adding a term that takes into account phonon drag as discussed in section 3.4. 


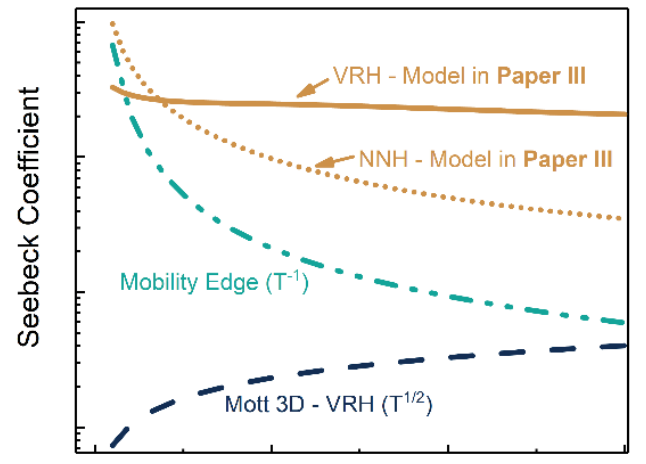

Temperature

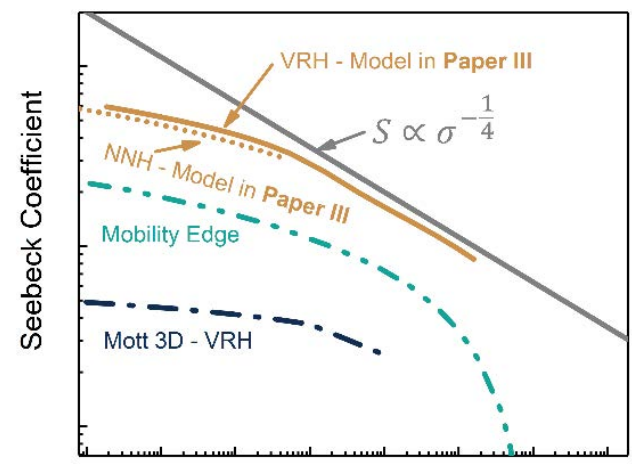

Conductivity

Figure 3.3 (left) Functional shape of temperature dependence of the Seebeck coefficient obtained from various models. (right) Functional shape of conductivity dependence obtained from the same models. The grey line indicates the empirical $-1 / 4$ power law. The model curves have been shifted vertically for improved readability and do not present any absolute trends of Seebeck coefficient or conductivity.

\subsubsection{Seebeck Coefficient from Percolation in a Gaussian DOS}

The expression for the Peltier coefficient in Equation (3.15) is easily adopted to percolation transport models by exchanging the mobility in Equation (3.13) for the number of electrical bonds per site energy $B(E)$ so that Equation (3.15) becomes ${ }^{13}$

$$
\Pi_{\mathrm{Perc}}=\frac{1}{q} \frac{\int\left(E-E_{F}\right) g(E) B(E) d E}{\int g(E) B(E) d E} .
$$

For a Gaussian DOS and assuming low temperatures this gives for the Seebeck coefficient a logarithmic $c^{-1}$ concentration dependence and includes the effect of the initial energetic disorder $\hat{\sigma}^{13}$

$$
S_{\text {Perc }}=-\frac{k_{B}}{q}\left[-\ln \left(\frac{1}{c}\right)+\frac{1}{2}\left(\frac{\hat{\sigma}}{k_{B} T}\right)^{2}-\frac{E_{\text {Perc }}^{*}}{k_{B} T}+\frac{3 \hat{\sigma}^{2}}{E_{\text {Perc }}^{*} k_{B} T}\right] .
$$

Also, this model predicts a logarithmic dependence on the inverse concentration that is offset by the energetic disorder and the transport energy. By extension it can be assumed that the conductivity dependence roughly follows $S_{\text {Perc }} \propto \ln \left(\frac{1}{\sigma}\right)$ or at least $S_{\text {Perc }} \propto \ln \left(\frac{1}{\sqrt{\sigma}}\right)$. 
Depending on disorder $\hat{\sigma}$ and transport energy $E_{\text {Perc }}^{*}$, the thermopower from Equation (3.28) changes sign at some concentration. The temperature dependence is close to the $T^{-1}$ relationship found for band-like transport, plotted on the left side of Figure 3.3. The inclination of the temperature dependence mainly depends on the amount of energetic disorder and increases with it.

\subsubsection{Seebeck Coefficient from the Mobility Edge Model}

The mobility edge model assumes transport to occur in a band of extended states above a certain energy (see section 2.2.2). Consequently the expression of the thermopower in Equation (S) includes a term $A$ to account for the distribution of states beyond the mobility edge ${ }^{10,12,14}$

$$
S_{M E}=\frac{k_{B}}{q} \frac{E^{*}-E_{F}}{k_{B} T}+A .
$$

The parameter $A$ is generally taken to be temperature independent and thus the Seebeck coefficient is proportional to $T^{-1}$.

Glaudell et al. approximated the energy difference between Fermi level and transport energy with the Arrhenius activation energy of the conductivity $E_{A c t}=B \ln (\sigma)+C$, where $B$ and $C$ are fit constants ${ }^{10}$. This substitution results in a logarithmic dependence of the Seebeck coefficient on the electrical conductivity,

$$
S_{M E}=\frac{B \ln (\sigma)+C}{T} .
$$

The functional shape temperature and conductivity dependence is plotted in Figure 3.4 together with the predictions from other models.

\subsubsection{Seebeck Coefficient from Mott's Variable Range Hopping Model}

From Mott's 3D variable range hopping in a constant DOS (see section 2.2.4) the Seebeck coefficient follows as ${ }^{14,15}$

$$
S_{V R H}=\frac{k_{B}^{2}}{2 q}\left(T_{0} T\right)^{\frac{1}{2}} \frac{\partial \ln (g(E))}{\partial E},
$$


with $T_{0}$ the characteristic temperature, which can be obtained from fitting the temperature dependence of the conductivity to the Mott law. Mott 3D VRH leads to a square root temperature dependence of the Seebeck coefficient and a logarithm-like conductivity dependence ${ }^{10}$. Especially the latter is not observed experimentally for doped systems, which is not surprising considering that this model requires a constant DOS at the Fermi energy with variation of charge carrier concentration, which has been shown not to be the case in Paper II and III as well as in previously published literature ${ }^{16-18}$ and sections (2.2.6) and (2.2.7).

3.3.6 Power-law Relationship between Seebeck Coefficient and Electrical Conductivity The thermopower from models describe thus far shows a logarithmic dependence on conductivity and is linear in temperature,

$$
S \propto \ln (\sigma) \quad \text { and } \quad S \propto T^{-1} .
$$

Both dependencies have been found experimentally for acid-doped polyaniline, propyrrole and perchlorate-doped polyacytelene ${ }^{7}$ and $\mathrm{PF}_{6}$ - doped $\mathrm{P} 3 \mathrm{HT}$ ( $0-34 \%$ relative dopant concentration) ${ }^{9}$, all of which exhibit variations of the inclination upon doping or stretching of the polymer film. While exhibiting a distinct $S \propto \ln (\sigma)$ relationship, $\mathrm{F}_{4}$ TCNQ-doped VOPc (0.2 - 2 mol-\%) only shows weak $T^{-1}$ temperature dependence, with little to none inclination change ${ }^{19}$. Thermopower investigations of OFETs reveal a logarithmic conductivity dependence and very little temperature dependence, irrespective of the material used, including experimental and numerical results in Paper $\mathbf{V}$ as well as measurements in Refs. ${ }^{20,21}$.

Interestingly, many doped ODSCs show a quasi-power-law relationship between the Seebeck coefficient and electrical conductivity that appears to be universal for several different host-guest material combinations and doping methods ${ }^{10,15}$. The relation and several data taken from literature as well as data from this thesis is plotted in Figure 3.4 and the power-law can be seen to roughly follow

$$
S \propto \sigma^{-\frac{1}{4}} \quad \text { or } \quad S^{2} \propto \sigma^{-\frac{1}{2}} .
$$

Hence, in order to increase the power factor $P F$ and thereby the figure of merit $Z T$ it is advantageous to increase the electrical conductivity rather than the Seebeck coefficient, despite the quadratic dependence on the thermopower in Equations (3.11) and (3.12). 


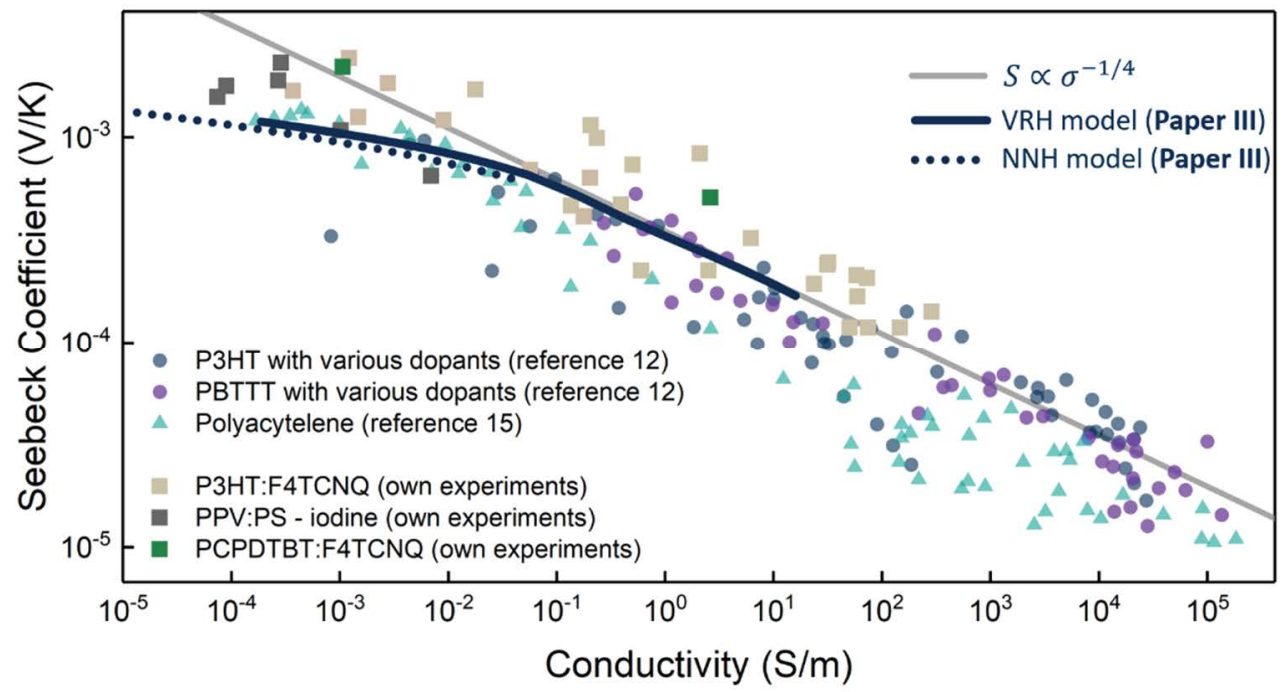

Figure 3.4 Compilation of experimental data from references ${ }^{10,22}$ of the conductivity dependence of the Seebeck coefficient exhibiting a universal-1/4 power-law relationship as indicated by the grey line. Solid and dotted lines are calculated with the analytical model described in Paper II and III with VRH and NNH, respectively.

The quasi-power-law relationship was first observed by Kaiser, however, without commenting on it ${ }^{15}$ and later by Glaudell et al. who were unsuccessful in fitting any of the existing transport models (Equations (3.28) (3.30) (3.31) ) to the experimental data and argued that the thermoelectric properties are dominated by the polymer and not the dopant ${ }^{10}$. Dongmin Kang et al. presented a model that describes Seebeck coefficient and conductivity by an empirically obtained general transport function that is discussed in the next paragraph ${ }^{23}$. In Paper III we were able to explain the power-law in Equation (3.33) by variable range hopping in a DOS that is modified by the presence of dopant ions using the model developed in Paper II.

\subsubsection{Seebeck Coefficient from a General Transport Function}

In Ref. ${ }^{23}$ Dongmin Kang et al. developed a generalized charge transport model where the conductivity is characterized by a single transport function and the Fermi-Dirac distribution $f$ 


$$
\sigma=\int \sigma_{E}\left(-\frac{\partial f}{\partial E}\right) d E
$$

with

$$
\begin{gathered}
\sigma_{E}(E, T)=\sigma_{E_{0}}(T)\left(\frac{E-E_{\text {trans }}}{k_{B} T}\right)^{s} \quad\left(E>E_{\text {trans }}\right) \\
\sigma_{E}(E, T)=0 \quad\left(E<E_{\text {trans }}\right) .
\end{gathered}
$$

Hence, carriers sitting below the transport edge $E_{\text {trans }}$ do not contribute to the conductivity and the exponent $s$ characterizes the distribution of states in energy above the transport edge. The Seebeck coefficient is then described by the energy dependence of the transport function in analogy to Equation (3.16)

$$
S_{G T}=\frac{1}{\sigma} \frac{k_{B}}{q} \int\left(\frac{E-E_{F}}{k_{B} T}\right) \sigma_{E}\left(-\frac{\partial f}{\partial E}\right) d E .
$$

The temperature-dependent transport coefficient $\sigma_{E_{0}}(T)$ is obtained from comparing temperature dependence measurements of conductivity and thermopower and using Equations (3.33) and (3.35) and has the form of an activated conductivity

$$
\sigma_{E_{0}}(T) \propto \exp \left[-\left(\frac{W_{\gamma}}{k_{B} T}\right)^{\gamma}\right] .
$$

Here $W_{\gamma}$ depends on the polymers morphology and is related to the choice of $\gamma$, which itself is related to the connectivity of a polymer ${ }^{24}$. The connections of the various parameters and exponents in this model to material properties is empirical and not founded on physical models. However, the general nature of the mathematics allows various other charge transport models to be expressed via the generalized transport function $\sigma_{E}(E, T)$ as shown in the supplementary information of Ref. ${ }^{23}$. Different classes of polymers are then identified by combinations of $s$ and $\sigma_{E_{0}}$ values based on their thermopower-conductivity relationship rather than being connected to any one type of charge transport mechanism.

\subsubsection{Our Contribution}

In Paper II we have developed a charge transport model, based on a previously developed model by Arkhipov et al. ${ }^{17,25}$, that describes charge transport by percolation in a DOS that is broadened and exponentially extended to lower energies 56 
by the presence of immobile dopant ions. In Paper III we used this model to distinguish between variable range hopping and nearest neighbour hopping based on the temperature and conductivity dependence of the experimentally obtained thermopower. The details of this model are described in in Paper II (for the nearest neighbour hopping case only) and Paper III (and the supplementary information of the latter two). The calculation of the Seebeck coefficient follows Equation (3.18), however, a crucial difference arises between NNH and VRH in the calculation of the transport energy $E^{*}$ as can be seen on the left side of Figure 3.5.
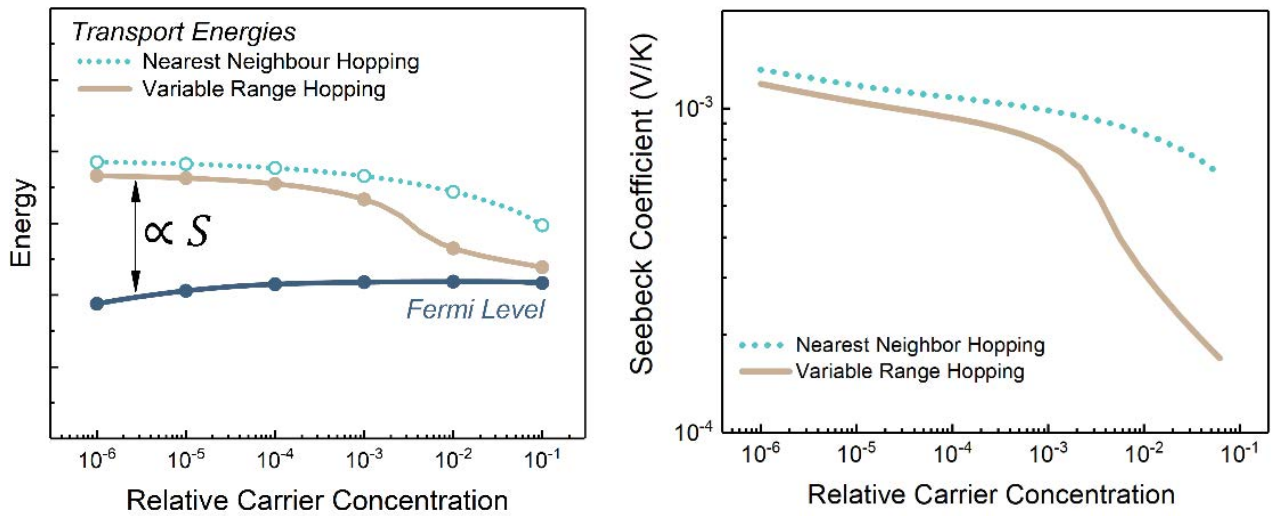

Figure 3.5 (left) Fermi and Transport energy as a function of charge carrier concentration in the case of NNH (dashed lines) and VRH (solid lines). (right) Distinct difference between NNH and VRH in the concentrations dependence of the Seebeck coefficient. Adopted with permission from Paper III.

The effect on the Seebeck coefficient is that at larger concentrations VRH leads to a faster drop of the Seebeck coefficient than NNH as can be seen on the right side of Figure 3.5. The functional shape of the concentration dependence of the conductivity, however, is similar for VRH and NNH. As can be seen in Figure 3.4 the stronger concentration dependence of $\mathrm{VRH}$ at higher concentrations leads to the experimentally observed $S \propto \sigma^{-\frac{1}{4}}$, which is not reproduced by NNH. This suggests that there is substantial amount of VRH for doped ODSCs even at room temperature. Without the dopant-induced modifications to DOS, the differences between VRH and $\mathrm{NNH}$ vanish and a stretched logarithmic behaviour is recovered for the conductivity dependence of the thermopower, such as predicted by the previously described models. Agreement of this model to the data compiled in Ref. ${ }^{10}$ was obtained by using reasonable material parameters for hopping frequency $\left(v_{0}=10^{11} \mathrm{~Hz}\right)$, localization length $(\alpha=2 \mathrm{~nm})$, nearest neighbour distance $\left(a_{N N}=1.8 \mathrm{~nm}\right)$, dielectric 
constant $\left(\varepsilon_{R}=3.6\right)$ and disorder $(\widetilde{\sigma}=50 \mathrm{meV})$. Within reasonable parameter changes, the main result of Paper III could not be changed and especially the initial disorder seems to have little influence on the curve in Figure 3.4 as shown in the SI of Paper III. This agrees with the universal experimental trend in Figure 3.4 for several different types of materials exhibiting different morphologies and energetic disorders. Additionally, no parameter combination was found that would create a -1/4 powerlaw dependence of the thermopower for the case of $\mathrm{NNH}$, which instead showed a much flatter (approximately -1/10) power-law. Further evidence for the consistency of the model in Paper III is given by the fact that the measured temperature dependence of the conductivity could only be reproduced by assuming VRH in a modified DOS.

\subsection{FINAL REMARK}

All of the models described above implicitly assume (typically via Equation (3.16)) that charge carriers only take their "own" energy with them and do not carry along any energy from carrier-phonon exchanges that might have occurred during hopping. This is typically referred to as phonon drag and the energy associated with it is proportional to a fraction $f$ of the charge carriers activation energy of the mobility $E_{\mu}$ and enters the Seebeck (and Peltier) coefficient by addition of a temperature dependent term ${ }^{6,9,27}$

$$
S=S_{\text {Model }}+f \frac{E_{\mu}}{k_{B} T}
$$




\subsection{BIBLIOGRAPHY}

1. Yu, C., Kim, Y. S., Kim, D. \& Grunlan, J. C. Thermoelectric behavior of segregated-network polymer nanocomposites. Nano Lett. 8, 4428-4432 (2008).

2. Bubnova, O. et al. Optimization of the thermoelectric figure of merit in the conducting polymer poly(3,4-ethylenedioxythiophene). Nat. Mater. 10, 429433 (2011).

3. Snyder, G. J. \& Toberer, E. S. Complex thermoelectric materials. Nat. Mater. 7, 105-114 (2008).

4. Lu, N., Li, L. \& Liu, M. A review of carrier thermoelectric-transport theory in organic semiconductors. Phys. Chem. Chem. Phys. 18, 19503-19525 (2016).

5. Cho, C. et al. Outstanding Low Temperature Thermoelectric Power Factor from Completely Organic Thin Films Enabled by Multidimensional Conjugated Nanomaterials. Adv. Energy Mater. 6, 1-8 (2016).

6. Fritzsche, H. A general expression for the thermoelectric power. Solid State Commun. 9, 1813-1815 (1971).

7. Mateeva, N., Niculescu, H., Schlenoff, J. \& Testardi, L. R. Correlation of Seebeck coefficient and electric conductivity in polyaniline and polypyrrole. J. Appl. Phys. 83, 3111-3117 (1998).

8. Emin, D. Pair breaking in semiclassical singlet small-bipolaron hopping. Phys. Rev. B 53, 1260-1268 (1996).

9. Xuan, Y. et al. Thermoelectric properties of conducting polymers: The case of poly(3-hexylthiophene). Phys. Rev. B 82, (2010).

10. Glaudell, A. M., Cochran, J. E., Patel, S. N. \& Chabinyc, M. L. Impact of the Doping Method on Conductivity and Thermopower in Semiconducting Polythiophenes. Adv. Energy Mater. 5, 1401072 (2015).

11. Emin, D., Seager, C. H. \& Quinn, R. K. Small-polaron hopping motion in some chalcogenide glasses. Phys. Rev. Lett. 28, 813-816 (1972). 
12. Park, Y. W., Denenstein, A., Chiang, C. K., Heeger, A. J. \& MacDiarmid, A. G. Semiconductor-metal transition in doped $(\mathrm{CH}) \mathrm{x}$ : Thermoelectric power. Solid State Commun. 29, 747-751 (1979).

13. Baranovskii, S. D., Zvyagin, I. P., Cordes, H., Yamasaki, S. \& Thomas, P. Percolation approach to hopping transport in organic disordered solids. Phys. Status Solidi Basic Res. 230, 281-287 (2002).

14. Mott, N. F. \& Davis, E. A. Electronic processes in non-crystalline materials. (Oxford University Press, 2012).

15. Kaiser, A. B. Electronic transport properties of conducting polymers and carbon nanotubes. Reports Prog. Phys. 64, 1 (2001).

16. Arkhipov, V. I., Heremans, P., Emelianova, E. V. \& Bässler, H. Effect of doping on the density-of-states distribution and carrier hopping in disordered organic semiconductors. Phys. Rev. B 71, 45214 (2005).

17. Arkhipov, V. I., Emelianova, E. V., Heremans, P. \& Bässler, H. Analytic model of carrier mobility in doped disordered organic semiconductors. Phys. Rev. B 72, 235202 (2005).

18. Efros, a L. \& Shklovskii, B. I. Coulomb gap and low temperature conductivity of disordered systems. J. Phys. C Solid State Phys. 8, L49-L51 (1975).

19. Pfeiffer, M., Beyer, A., Fritz, T. \& Leo, K. Controlled doping of phthalocyanine layers by cosublimation with acceptor molecules: A systematic Seebeck and conductivity study. Appl. Phys. Lett. 73, 3202 (1998).

20. Venkateshvaran, D. et al. Approaching disorder-free transport in high-mobility conjugated polymers. Nature 515, 384-388 (2014).

21. Pernstich, K. P., Rössner, B. \& Batlogg, B. Field-effect-modulated Seebeck coefficient in organic semiconductors. Nat. Mater. 7, 321-325 (2008).

22. Kaiser, A. B. Electronic transport properties of conducting polymers and carbon nanotubes. Reports Prog. Phys. 64, 1-49 (2001).

23. Dongmin Kang, S. \& Jeffrey Snyder, G. Charge-transport model for conducting polymers. Nat. Mater. 1, 1-7 (2016). 
24. Urban, J. J. Organic electronics: One model to rule them all. Nat. Mater. 16, 157-159 (2016).

25. Arkhipov, V. I., Heremans, P., Emelianova, E. V. \& Bässler, H. Effect of doping on the density-of-states distribution and carrier hopping in disordered organic semiconductors. Phys. Rev. B 71, 45214 (2005).

26. Abdalla, H., Zuo, G. \& Kemerink, M. Range and energetics of charge hopping in organic semiconductors. Phys. Rev. B 96, 241202 (2017).

27. Emin, D. Thermoelectric Power Due to Electronic Hopping Motion. Phys. Rev. Lett. 35, 882-885 (1975). 



\section{ORGANIC FIELD EFFECT TRANSISTORS}

\subsection{WORKING PRINCIPLE}

Embedding an organic semiconducting material into a field effect structure such as in Figure 4.1 allows for the film to be doped electrostatically without the introduction of dopant ions and the effects associated with it (see sections 2.2.6 and 2.2.7 and 3.3.8). While FETs with inorganic active layers find wide commercial use, OFETs are still mainly used for the purpose of research. One of the reasons for this is the field effect mobility $\mu_{F E}$ which until recently lagged behind amorphous Silicon, but is catching up with mobilities of more than $1 \mathrm{~cm}^{2} / \mathrm{Vs}$ reported consistently ${ }^{1-4}$. However, for many application higher mobilities are still needed.

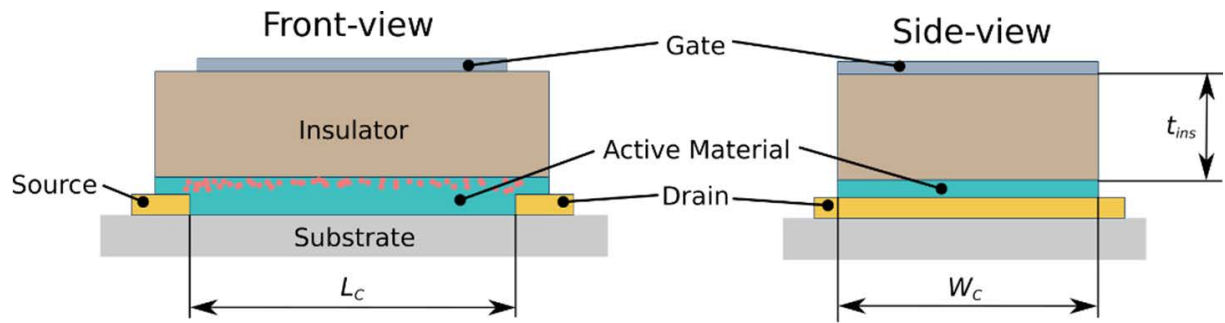

Figure 4.1 Top-gate bottom contact OFET on top of a substrate. The width and length of the channel as well as the thickness of the insulator are indicated.

A top-gate bottom-contact OFET is shown in Figure 4.1, however, other device structures are possible. In each case, the active layer is contacted by a source and a drain contact. The gate contact is electrically separated from the rest of the structure by an insulating layer, which is typically PMMA or Cytop in top-gate configuration or $\mathrm{SiO}_{2}$ in bottom-gate configuration.

Since intrinsic ODSCs have only very few inherent free charge carriers, the generation of mobile charges in OFETs occurs via accumulation of charges in the channel. The application of a gate voltage $V_{G}$ between the gate contact and the source or drain contact accumulates mobile charges in the channel between the contacts. The charge accumulation density decreases with distance from the semiconductor/insulator 
interface as shown on the left side of Figure 4.2. Especially at large gate fields and if morphology is favourable, most charge carriers will be confined to a quasi-twodimensional (quasi-2D) layer at the interface, as we have shown numerically and experimentally in Paper II. However, in some literature a 3D distribution is assumed.
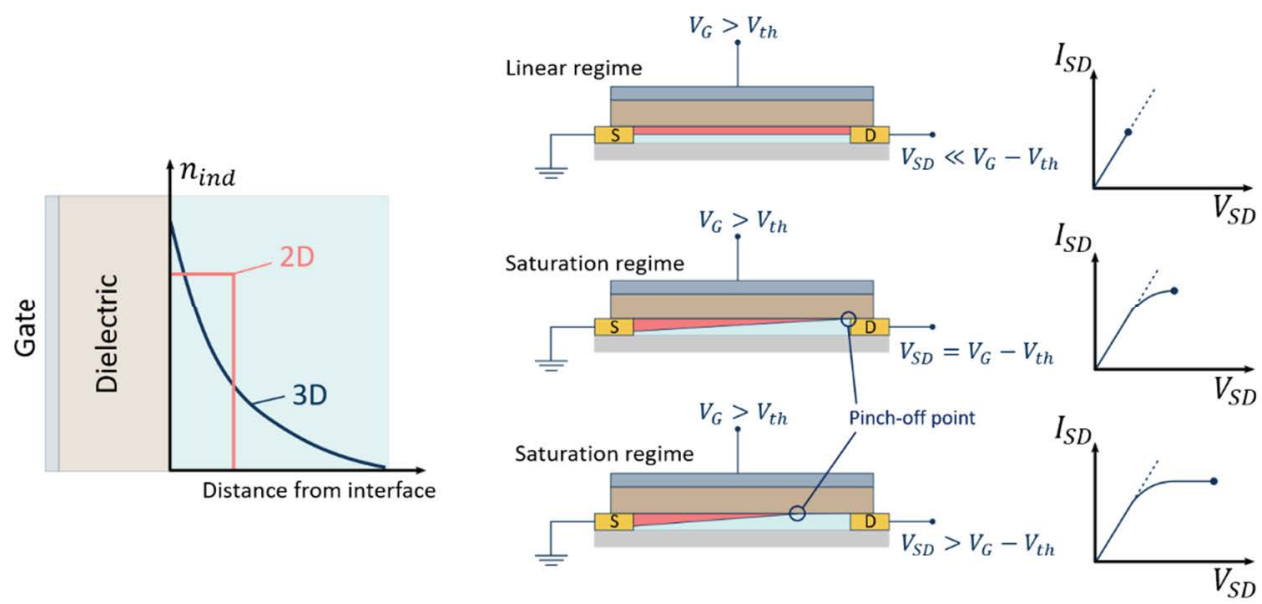

Figure 4.2 (left) Three- and two-dimensional distribution of charge carriers in the channel of a FET. (right) Different modes of operation and corresponding transfer characteristics.

The total induced areal charge carrier density assuming 2D charge distribution is calculated from Gauss's law and is a function of $V_{G}$ and the gate capacitance $C_{G}$

$$
n_{\text {ind }}=\frac{1}{q} C_{G} V_{G}=\frac{1}{q} \varepsilon_{0} \varepsilon_{r} F_{G},
$$

with the gate field $F_{G}=V_{G} / t_{i n s}$ and $t_{\text {ins }}$ the thickness of the insulating layer, indicated in Figure 4.1. The induced carrier density $n_{\text {ind }}$ is typically limited to $10^{16} \mathrm{~m}^{-2}$ to $10^{17} \mathrm{~m}^{-2}$ due to the relatively small gate capacitance achievable with polymeric materials and the limitation of breakthrough voltage of the same. Higher gate capacitances and thus charge carrier concentrations can be achieved by using an electrolyte as gate insulator ${ }^{5}$. However, in-diffusion of ions from the electrolyte to the semiconductor can lead to electrochemical doping of the film ${ }^{6}$. While this may be intended in some cases, it is generally avoided by using single crystalline molecular materials as active layer such as rubrene that prevent in-diffusion ${ }^{7}$.

ODSCs often have some density of deep traps (deep w.r.t to the available thermal energy to activate the hopping process) that need to be filled before any transport 64 
can occur. Hence, mobile carriers are only accumulated once $V_{G}$ is above a threshold voltage $V_{t h}$, the magnitude of which depends on the material and device structure. Another cause for the presence of a threshold voltage are ionic effects of the dielectric interface that offset the gate voltage.

The field effect mobility $\mu_{F E}$ can be extracted from the transfer characteristics of source-drain current $I_{S D}$ as a function of source-drain voltage $V_{S D}$ and gate voltage $V_{G}$. Depending on the magnitude of $V_{S D}$, two charge transport regimes are distinguished. In the linear regime, plotted on the right side of Figure 4.2, $V_{S D}<<$ $V_{G}-V_{T}$ and the effect of the source-drain field on the gate field is small, leading to a uniformly distributed charge carrier concentration throughout the channel (distribution perpendicular to the interface may still vary with distance). The current is then calculated as

$$
I_{S D}^{l i n}=\mu_{F E} \frac{W}{L} C_{G}\left(V_{G}-V_{T}\right) V_{S D}
$$

where $W$ and $L$ are the width and length of the channel, respectively. In the saturation regime, depicted in Figure 4.2, where $V_{S D}>V_{G}-V_{T}$, the channel is partially pinched-off by the source-drain field and the current begins to saturate and to become independent on the source-drain voltage

$$
I_{S D}^{s a t}=\mu_{F E} \frac{W}{L} C_{G}\left(V_{G}-V_{T}\right)^{2} .
$$

At high enough $V_{S D}$ the channel is pinched-off completely at the source electrode and the current is saturated, seen in Figure 4.2. Increasing $V_{S D}$ shifts the pinch-off point closer to the drain electrode and hence, care must be taken in designing the channel length in a FET structure to not make it too small. The corresponding field-effect mobilities can then be extracted from the transfer characteristics,

$$
\begin{gathered}
\mu_{F E}^{l i n}=\frac{L}{C_{G} W V_{D}} \frac{\partial I_{d}^{l i n}}{\partial V_{G}} \\
\mu_{F E}^{\text {sat }}=\frac{L}{C_{G} W V_{D}} \frac{1}{\left(V_{G}-V_{t h}\right)} \frac{\partial I_{d}^{s a t}}{\partial V_{G}},
\end{gathered}
$$

where $\partial I_{d}^{l i n} / \partial V_{G}$ is the transconductance. The threshold voltage is determined by plotting $\sqrt{I_{S D}}$ in the saturation regime as a function of $V_{G}$ and finding the intersection 
with the $x$-axis by interpolation of the linear fit. In numerical simulations the threshold voltage is zero by definition.

\subsection{Charge TRANSPORT IN OFETS}

Early on, the concentration dependence of ODSCs at high concentrations was successfully described with a multiple trap and release (MTR) model where transport occurs by thermal excitation of a carrier from a trap to a mobility edge above which states are extended, such as described in section 2.2.2. The trap distribution was originally assumed to be double exponential and was successful in describing temperature and gate field dependence of various oligothiophene films ${ }^{8}$ and was later shown to also reproduce transfer characteristics for $\mathrm{P} 3 \mathrm{HT}$ and poly(thiophenes) films, where a single exponential distribution of traps was assumed ${ }^{9}$. While the MTR model is well suited to describe charge transport in (semi-)crystalline organic semiconductors, where evidence for the existence of extended states has been found 10,11 , it does not necessarily apply to amorphous films, where transport can occur between trap states alone ${ }^{12}$ (see also section 2.2.3).

To address this issue Vissenberg-Matters (VM) developed a model that describes transport in OFETs with percolation theory by 3D variable range hopping in an exponential DOS ${ }^{12}$. Hopping is activated from the Fermi level $E_{F}$ to a transport energy $E^{*}$. A key assumption of the VM model is that carriers are distributed, per the FermiDirac distribution, mainly at low energy sites in the strongly energy-dependent tail of the DOS, so that the DOOS has a Fermi level

$$
E_{F} \ll k_{B} T_{0}
$$

with $k_{B} T_{0}$ the characteristic energy of the trap distribution. In this scenario carriers prefer to hop to higher energies (rather than larger distances) due to the exponentially larger number of available states further up in the DOS. This limits the validity of this model to temperatures $T<T_{0}$. Above this limit the energy dependence of the DOS is too weak and Mott-type behaviour is observed. The derivation of the conductivity $\sigma$ follows percolation theory and leads to an activated temperature behaviour and a super-linear dependence on induced carrier concentration $c$, 


$$
\sigma(c, T)=\sigma_{0}\left(\frac{\left(\frac{T_{0}}{T}\right)^{4} \sin \left(\frac{\pi T_{0}}{T}\right)}{(2 \alpha)^{3} B_{C}}\right)^{\frac{T_{0}}{T}} c^{\frac{T_{0}}{T}},
$$

with $\sigma_{0}$ being a prefactor to the conductivity. The increase in conductivity with concentration is due to state-filling of the tail states and thus dependent on the characteristic width of the DOS w.r.t. to the temperature.

\subsubsection{Dimensionality of Charge Transport}

The VM model explicitly assumes a 3D distribution of carriers in the film (see left side of Figure 4.2) and thus also a distribution of conductivities along the thickness $t$ of the material. This changes the expression for the linear current in Equation (4.2) to

$$
I_{S D}=\frac{W V_{S D}}{L} \int_{0}^{t} d z \sigma(c(z), T) .
$$

If Equations (4.7) and (4.8) are explicitly solved for a 3D distribution of carriers and the result expanded to a Taylor series, the source-drain current can be approximated as having a power-law dependence in gate voltage ${ }^{13}$

$$
I_{S D}^{3 D} \propto\left(V_{t h}-V_{G}\right)^{\frac{2 T_{0}}{T}-1} .
$$

Assuming a two-dimensional (2D) charge carrier distribution in form of a step function with a certain width (left side of Figure 4.2), the current shows a different power-law,

$$
I_{S D}^{2 D} \propto\left(V_{t h}-V_{G}\right)^{\frac{T_{0}}{T}} .
$$

Brondijk et al. used the difference in exponents between the 3D and 2D case to analyse the extent of carrier confinement in OFETs, by fitting a power-law to the gate voltage dependence of the source-drain current measured at different temperatures, depicted on the left side of Figure $4.3^{13}$. On the right side of Figure 4.3, the obtained exponent $\gamma$ is then plotted as a function of the inverse temperature $1000 / T$, which allows to determine the intersection with the $\gamma$ axis by extrapolation to $T \rightarrow \infty$. Per Equation (4.9), an intersection at -1 indicates 3D transport while an intersection at zero indicates 2D transport (Equation 4.10). In this way the charge transport in OFETs with a single-monolayer of T6 and self-assembled monolayer FETs (SAMFETs), which are inherently 2D, was found to be $2 \mathrm{D}$ while the transport in thicker films of $\mathrm{P} 3 \mathrm{HT}$, 
MDMO-PPV and PTV was found to be 3D. The slope on the right side of Figures 4.3 gives the characteristic width $T_{0}$ of the exponential DOS.
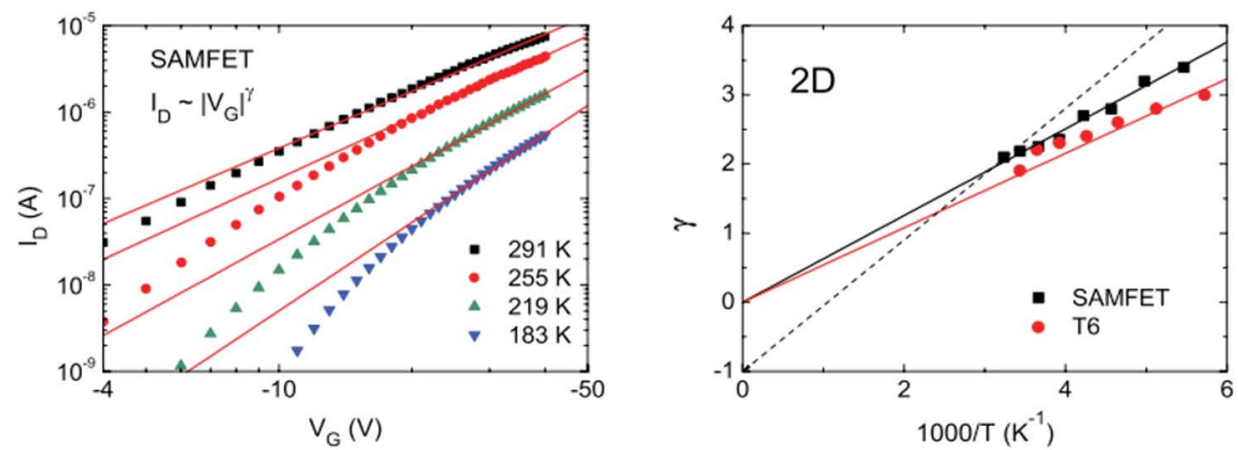

Figure 4.3 (left) Experimental transfer curves of a SAMFET measured as a function of temperature, at a drain bias of $V_{D}=-2 \mathrm{~V}$. The red lines are a power-law fit at high gate bias, for each temperature. (right) The exponent extracted from (left) versus inverse temperature. Adapted with permission from Brondijk et al ${ }^{13}$.

This extended-VM method of analysing charge transport dimensionality has subsequently been adopted to the saturation regime with $T_{0}$ values extracted in this way corresponding to the Urbach energy from absorbance measurements ${ }^{14}$.

In fact, the spatial extent of the charge carrying channel in OFETs has been subject to discussion since their inception. Experimental studies often focus on the thickness dependence of the mobility of active layers exhibiting long range order mostly found that it saturates after 2 to 5 monolayers (MLs) ${ }^{15-18}$. Other studies find that charge transport transitions from 2D to 3D after a thickness of about $10 \mathrm{~nm}$ by providing an alternative transport path through the upper layer of the film ${ }^{19}$ or that charge transport is inherently $3 \mathrm{D}$ altogether ${ }^{13}$.

\subsubsection{Our Contribution}

In Paper IV we have investigated the dimensionality of OFET transport by means of kinetic Monte Carlo simulations and mobility investigation of individual $3 \mathrm{~nm}$ Langemuir-Schäfer (LS) MLs of (P(NDI2OD-T2) (N2200) stacked to a thickness of 1 to 5 MLs. N2200 is a high-mobility n-type polymer with predominantly face-on orientation of the individual polymer backbones and is thus particularly well suited for the assembly into a well-defined ML. We find that the dimensionality tends to be inherently quasi-2D at high gate voltages due to the confinement of the charge carriers by the gate field. In the case of LS N2200 layers, this confinement is 
morphologically enhanced by the smaller inter-site distance in the direction of charge transport compared to the direction perpendicular to it.
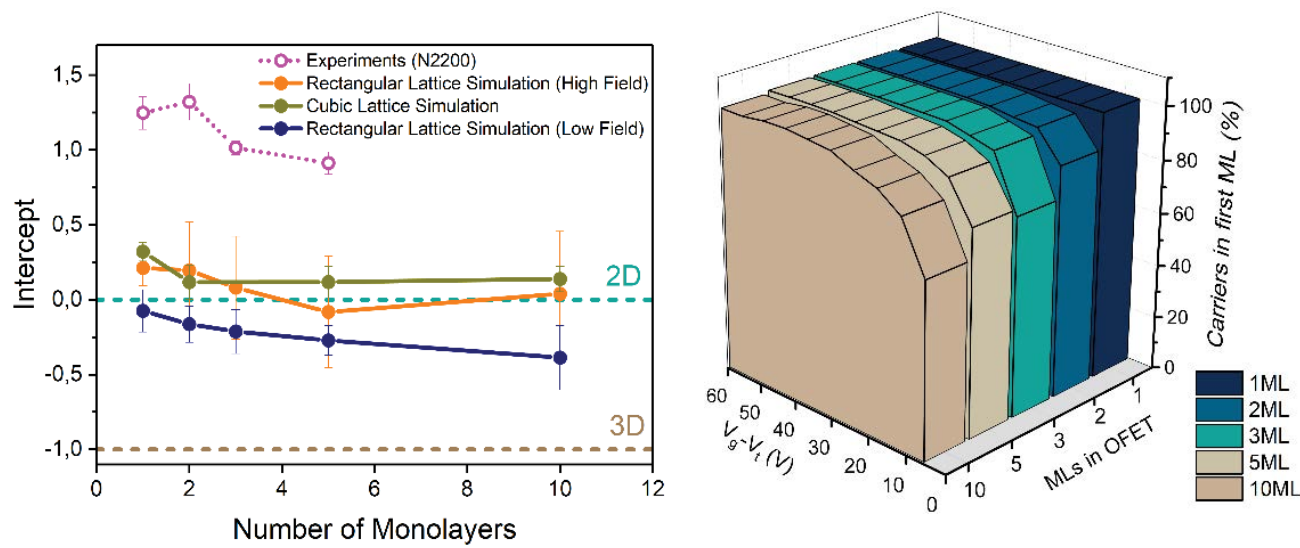

Figure 4.4 (left) Intercepts of $\gamma$ as a function of number of monolayers for experiments on N2200 OFETs and kinetic Monte Carlo simulations. (right) Percentage of carriers in the first monolayer as a function of gate voltage and total number of MLs in the device for a rectangular lattice. Adapted with permission from Paper IV.

By using the extended VM dimensionality analysis proposed by Brondijk et al. we find that unbiased linear fitting of the temperature dependence of $\gamma$ does not lead to a change of the interception from $2 \mathrm{D}(\gamma(0)=0)$ to $3 \mathrm{D}(\gamma(0)=-1)$ when increasing the number of MLs from $1 \mathrm{ML}$ to $5 \mathrm{ML}$. Rather, the intercept changes between 1 and 1.5, values that indicate sub-2D transport (left side of Figure 4.4). The transport in MCsimulated OFETs is found to be quasi-2D, independent of the number of MLs as indicated by the VM-type analysis and by simply looking at the position of the carriers in the simulation on the right side of Figure 4.4. The extracted values for the width of the DOS are, however, inconsistent with the input to the simulations. The disorder values for the experiments were found to be unphysically low, which has previously been observed for other materials fitted with the VM model ${ }^{20}$. 


\subsection{CONCENTRATION DEPENDENCE OF THE MOBILITY}

The field-effect mobility is expressed according to the VM model (Equation (4.3)) as ${ }^{21}$

$$
\mu_{F E}(c, T)=\frac{\sigma_{0}}{q}\left(\frac{\left(\frac{T_{0}}{T}\right)^{4} \sin \left(\frac{\pi T_{0}}{T}\right)}{(2 \alpha)^{3} B_{C}}\right)^{\frac{T_{0}}{T}} c^{\frac{T_{0}}{T}-1} .
$$

Note that the concentration dependence of the mobility increases with disorder (see Figure 4.5). This equation was initially used to describe the temperature and concentration dependence of PTV and pentacene ${ }^{12}$ and has later been shown to also describe transport in P3HT and a PPV-derivative ${ }^{21}$. Interestingly, if the same materials are characterized at low concentrations, in a diode configuration, the mobility was found to be orders of magnitude lower and constant in carrier concentration. The transition between these two regimes is plotted in Figure 4.5 and has been studied first by Tanase et al. ${ }^{21}$.

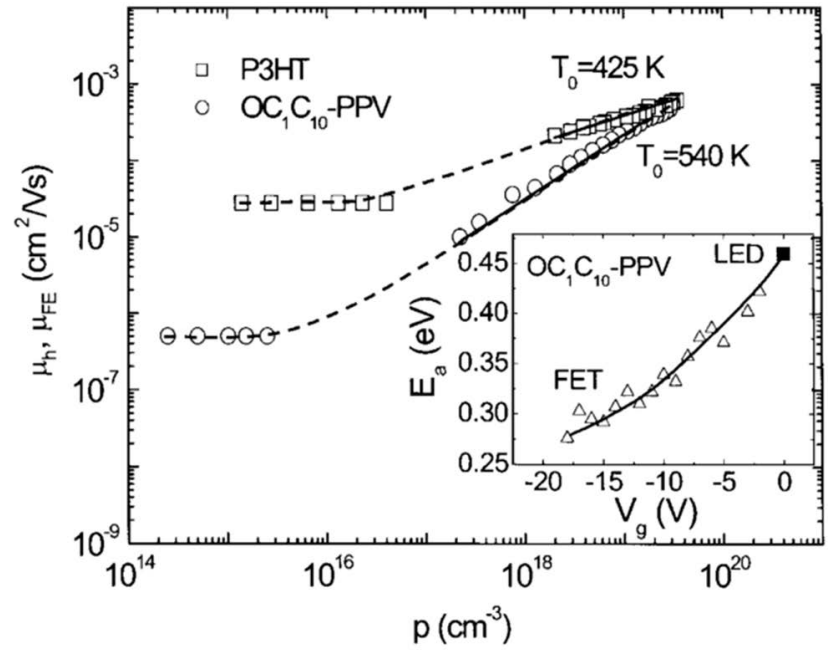

Figure 4.5 Mobility as a function of hole density $p$ in a diode and field-effect transistor for P3HT and $\mathrm{OC}_{1} \mathrm{C}_{10}$-PPV [measurements (symbols) and Equation (4.11) (lines)]. The dashed line is a guide to the eye. Inset: The activation energy of the mobility in the $O C_{1} C_{10}-P P V$ based FET as a function of gate voltage (triangles), together with the activation energy of $0.46 \mathrm{eV}$ as obtained from the diode at low densities (square). Adapted with permission from Tanase et al. ${ }^{21}$. 
The inset in Figure 4.5 shows the activation energy of the field-effect mobility obtained from Equation (4.11), which extrapolates remarkably well to the activation energy obtained with the correlated Gaussian disorder model (cGDM) at low concentrations. Additionally, by comparing $T_{0}$ values obtained from fitting the VM model to the high concentration regime to the disorder obtained from the cGDM, it was argued that the main reason between the discrepancy in mobility between diode and FET configuration is the large difference in carrier concentration and that the exponential DOS is a good approximation of the tail states of the Gaussian DOS. The apparent concentration independence at small concentration might be related to experimental and device-related factors playing a larger role in this regime.

Later experimental investigations of semi-crystalline polymer materials $\mathrm{P} 3 \mathrm{HT}$ and PQT-12 showed a reduced concentration dependence and fits with the VM model resulted in unphysically small disorders. It was found that the results were better described by the ME model with an exponential DOS ${ }^{22}$. Like in the VM model, in the ME model a smaller energetic disorder relates to a smaller concentration dependence and was attributed to a more crystalline morphology ${ }^{9}$. The same correlation between mobility, concentration dependence and disorder also follows from a variety of other disorder models ${ }^{23,24}$. In general, most models predict functionally equivalent concentration and temperature dependencies of the field-effect mobility and justification for choosing one model over another are usually done by correlating the material parameters (foremost the energetic disorder) extracted from model fits to experimental data to morphological investigations (usually crystallinity), allowing for some unavoidable degree of freedom in determining the charge transport mechanism or shape of DOS. 


\subsection{Transport at High Relative Concentrations}

Di Pietro et al. studied how different degrees of crystallinity effect the field-effect mobility of N2200 OFETs ${ }^{25}$. By varying the polarity of the solvent and post-processing treatments, the size of crystallites could be controlled. The measured concentration dependence of the mobility increases with increasing magnitude of the mobility, which is typically not observed in other literature. Furthermore, the increase in mobility correlates with crystallite size, i.e. degree of crystallinity. Since the simultaneous increase of both aspects of the mobility cannot be explained with classical disorder-based models, such as the VM model (Equation (4.11)), the ME model or any GDM, the authors suggested a model where Coulomb repulsion between charge carriers in doubly occupied crystallites dominates over disorder. In films with higher degree of crystallinity (and hence overall fewer number of crystallites), the charge carrier concentration at high gate voltages is in the order of the number of crystallites, which may then have a finite possibility of being occupied by two carriers, as shown schematically in Figure 4.6. The Coulomb repulsion between carriers in a doubly occupied domain raises their energy and allows one of them to easier overcome the energy barrier associated with amorphous regions in-between crystalline regions, thus improving charge transport. The inclusion of the Coulomb interaction effectively modifies the DOS of the material and may result in a disorder that is different from the inherent value of the material.

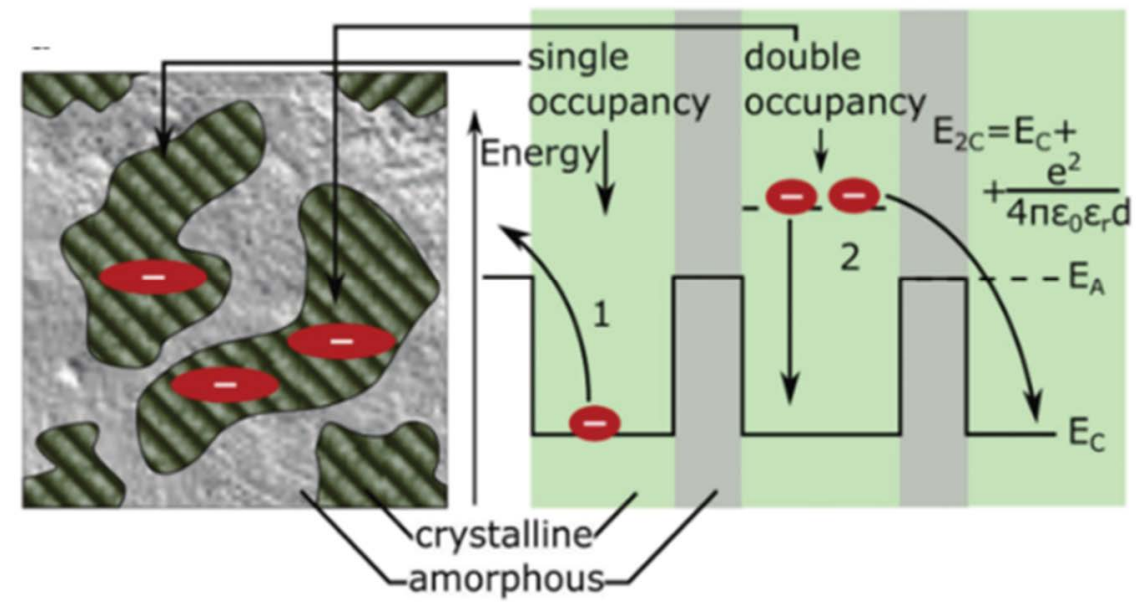

Figure 4.6 Single and double occupation of crystallites surrounded by an amorphous matrix. Adapted with permission from Di Pietro et al. ${ }^{25}$. 
Using electrolytes with high ionic but low electronic conductivities as gate insulators increases the gate capacitance, and thereby the induced carrier concentration by the formation of an electric double layer at the semiconductor/insulator interface that locally increases the gate capacitance, as schematically depicted on the left side of Figure 4.7. In the work of Xia et al. this effect was used to investigate the mobility dependence of rubrene single crystals gated by $\left[(\mathrm{EMIM})^{+}(\mathrm{TFSI})^{-}\right]^{7}$. Varying the gate voltage led to a peak in the source-drain current, shown in the upper panel of the right side of Figure 4.7 and a subsequent negative transconductance above a certain gate voltage, despite a linearly increasing hole concentration. Consequently, the mobility exhibits a peak sitting at a concentration corresponding to approximately 0.15 holes per rubrene molecule (assuming a molecular density of $1.9 \times 10^{14}$ molecules $\left(\mathrm{cm}^{2}\right)$. To explain their findings the authors proposed a model in which the negatively charged immobile ions in the electrolyte trap mobile charges in the semiconductor layer thereby reducing percolation and forcing carriers to take alternative and less efficient paths through the $2 \mathrm{D}$ channel.
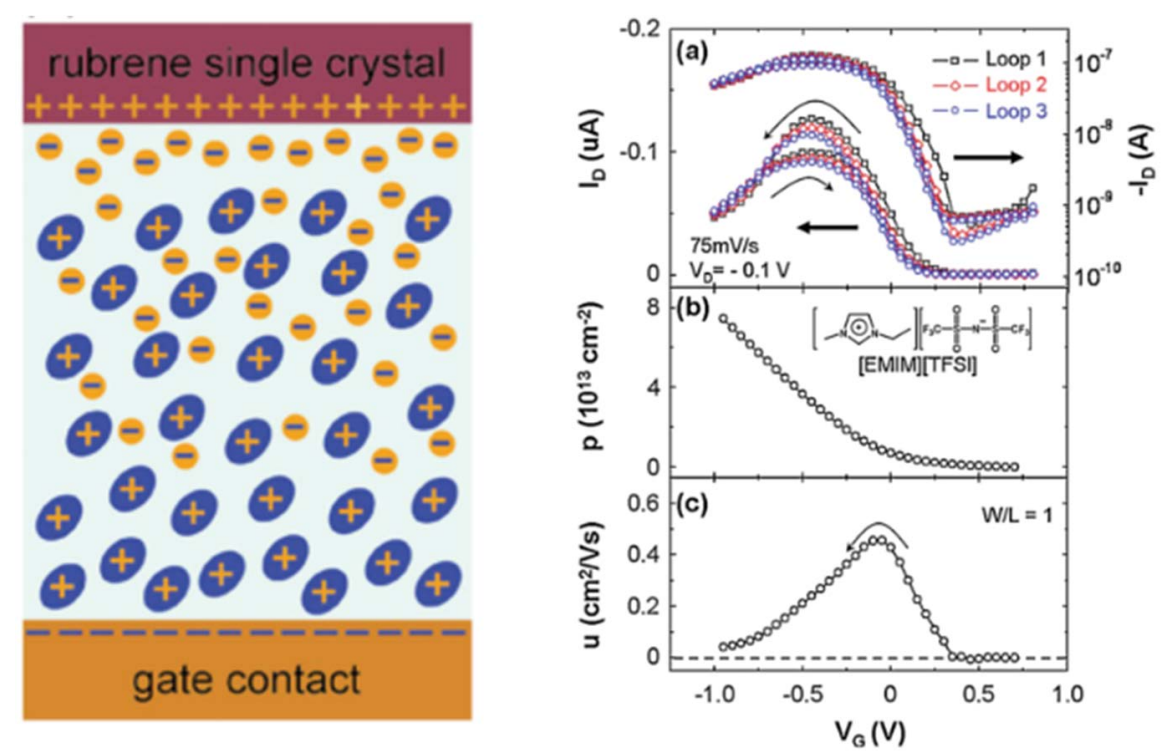

Figure 4.7 (left) Scheme of the channel charge and ion distribution. (right) Electrical characterization of a rubrene single-crystal EDLT. (a) ID-VG characteristics for three consecutive VG sweeps, acquired at the rate of $75 \mathrm{mV} / \mathrm{s}$, at a fixed drain bias. (b) Gate-induced hole density versus $V_{G}$, determined from capacitance-voltage measurements. (c) Mobility versus $V_{G}$. The inset in (b) shows the molecular structure of the ionic liquid used as the gate dielectric. Adapted with permission from Xia et al. 7 . 


\subsubsection{Our Contribution}

In Paper VI we study a range of transport phenomena related to the interplay between inter-carrier Coulomb interactions and multiple occupation in OFETs at high charge carrier concentrations. Two effects, however, stand out since they offer alternative but related explanations to the two phenomena discussed in section 4.4.

1) At a certain ratio between strength of inter-carrier Coulomb interaction and size of the on-site barrier $E_{B}$ for double occupation, we find an increase in magnitude and concentration dependence of the mobility, where concentration dependence decreases with smaller on-site barriers. This is similar to the results obtained by Di Pietro et al. for different degrees of crystallinity. From an investigation of the DOS and DOOS, we found the decreasing concentration dependence to be related to an increase of double occupation of low-energy tail states which reduces state-filling and its positive effect on the mobility. In fact, upon inspecting the modifications of the DOS it becomes clear that the deviating magnitude-concentration dependence relationship of the mobility concurs with the opening of a soft Coulomb gap, following the form suggested by Efros and Shklovskii described in section 2.2.8. The Coulomb gap decreases with decreasing inter-carrier Coulomb interactions (increasing intersite distance) as does the effect of a decreasing magnitude and concentration dependence of the mobility.

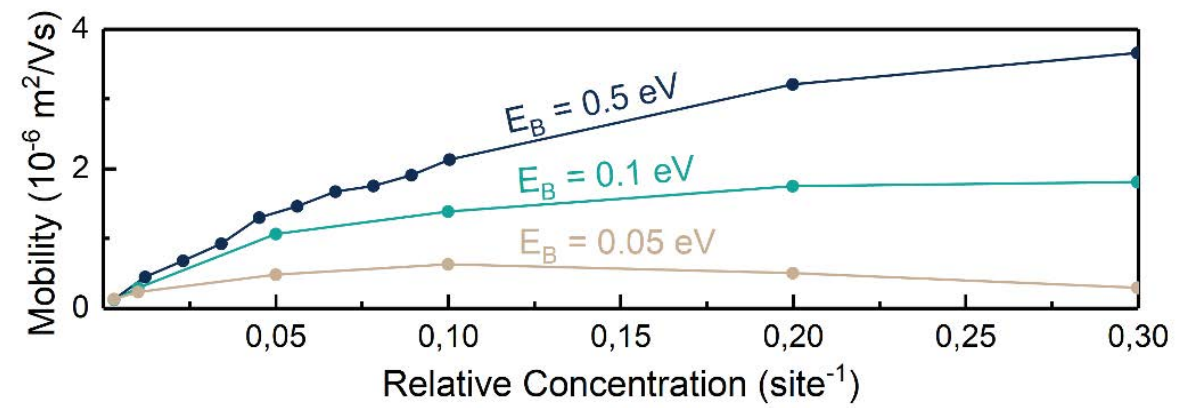

Figure 4.8 Increasing concentration dependence and magnitude of the mobility for increasing on-site barrier $E_{B}$ calculated with the kinetic Monte Carlo algorithm described in section 5 .

2) For larger unit cells, where inter-carrier Coulomb interactions are smaller, the numerical simulations show a collapse of the mobility at per-site concentrations of integer multiples of $100 \%$ followed by a subsequent recovery. This is attributed to the formation of a Mott-Hubbard Coulomb gap. With increasing strength of Coulomb interactions (smaller inter-site distances) or decreasing on-site barrier the mobility 74 
peak shifts to smaller concentrations and is furthermore characterized by a later recovery of the mobility or absence of it. Using the lattice parameters from Xia et al. as input parameters to the $M C$ simulations, we were able to accurately reproduce the mobility peak observed in their work, thus offering a different explanation to the one given in Ref. ${ }^{7}$.

\subsection{THERMOELECTRIC PROPERTIES OF OFETS}

Contrary to doped materials, only little literature exists on thermoelectric investigations of OFETs, with the first report on the Seebeck coefficient of Pentacene FETs in $2007{ }^{26}$.

Nonetheless, thermoelectric investigations can of course also give additional insight in to charge transport of OFETs by providing information about the transport energetics and thus aid identification of the transport mechanism (section 3.2). This approach was taken by Pernstich et al. who measured the Seebeck coefficient of Rubrene single crystal and Pentacene thin film FETs as a function of induced concentration and temperature ${ }^{27}$. The Seebeck coefficient showed a logarithmic dependence on the concentration and by fitting the temperature dependence to the expression for the Seebeck coefficient in the ME model (Equation (3.31)) the transport constant $A$ was obtained (The values for $E_{F}$ and $E^{*}$ have been obtained by a different model). The values for the constant $A$, which essentially describes the DOS above the ME, were found to be within the range of those found for inorganic crystalline semiconductors, thus indicating band-like transport characterized by scattering processes. An investigation of the mobility was, however, absent.

As discussed in section 4.3 there was believed to be a strong correlation between crystalline morphology, low energetic disorder and high field-effect mobility. This correlation, however, was interrupted by the discovery of donor-acceptor copolymers with near amorphous morphologies and mobilities in the range of or even higher than (semi-) crystalline polymers ${ }^{1,28}$. This apparent contradiction of previous results was subsequently resolved by Venkateshvaran et al. through a combined thermoelectric and morphological study comparing the structurally unordered polymers IDTBT, PSeDPPBT and DPPTTT to PBTTT, a semi-crystalline reference system ${ }^{29}$. The Seebeck coefficient showed a logarithmic dependence on induced charge carrier concentration, where the slope was found to be related to the density of traps, 
with less energetically disordered systems exhibiting smaller slopes. This prediction was corroborated by measurements of the Urbach energy by thermal deflectance measurements, which showed that the crystalline polymer PBTTT exhibits the highest energetic disorder ( $40 \mathrm{meV}$ ), while IDTBT was found to have the smallest energetic disorder ( $20 \mathrm{meV}$ ) despite the absence of long-range order, thus restoring the relationship between high mobility and low energetic disorder. A combination of molecular dynamics simulation and Raman spectroscopy related this finding to a strong resilience of the polymer backbone to conformational deformations.

All available literature data to date on the Seebeck coefficient of OFETs, including data in Paper V, shows a negative logarithmic dependence on charge carrier concentrations and conductivity, as predicted by most charge transport models discussed in chapter $3^{27,30,31}$. However, as discussed in section 3.3.6 many doped organic polymers, including materials that have been investigated in OFET geometries such as P3HT and PBTTT, exhibit a power-law relationship between Seebeck coefficient and conductivity ${ }^{32,33}$. One report directly compares thermoelectric properties of P3HT, PBTTT as well as of two n-type naphthalene diimides derivatives in OFET geometry and chemically doped films ${ }^{31}$. The authors find a logarithmic dependence on the conductivity in both cases, contrary to previously published results for doped P3HT and PBTTT. However, in this study PBTTT was doped using $\mathrm{NOPF}_{6}$ that has previously been shown to behave differently than other dopants and to deviate from the power-law and instead create an $S \propto \ln (\sigma)$ relationship when used to chemically dope $\mathrm{P}_{3} \mathrm{HT}^{33,34}$.

\subsubsection{Our Contribution}

OFETs generally exhibit charge carrier concentrations in the range of $10^{15} \mathrm{~m}^{-2}$ to $10^{17}$ $\mathrm{m}^{-2}$ leading to pairwise inter-carrier Coulomb energies ranging from $17 \mathrm{meV}$ to 127 $\mathrm{meV}$. These energies are greater than or at least in the range of the typical values for the width of the DOS of around $30 \mathrm{meV}$ to $50 \mathrm{meV}$ and $25 \mathrm{meV}$ thermal energy at room temperature. Hence, these Coulomb interactions should have an influence on the energetics and thus also on charge transport and cannot be ignored in the description thereof. In Paper $\mathbf{V}$ we investigate the effect of Coulomb interactions on charge and energy transport by $\mathrm{MC}$ simulations and compare our results to own experiments and all available literature data. We find that also simulated OFETs exhibit a logarithmic dependence of the Seebeck coefficient on induced charge carrier density and conductivity. The slope of both dependencies increases with the initial energetic disorder of the material, which qualitatively agrees with the findings of 
Venkateshvaran et al. ${ }^{30}$ rather than Pernstich et al. ${ }^{27}$. Upon removing inter-carrier Coulomb interactions in the simulations, conductivity as well as Seebeck coefficient are increased, contrary to the common inverse relationship between these two parameters. By investigating the variations of the energy levels involved (Equation (3.20)) we find that the reason for this behaviour is that broadening of the DOOS move the Fermi level closer to the transport energy, thereby reducing the Seebeck coefficient. At significantly higher concentrations, the effects of Coulomb interactions also lead to modifications of the DOS, which are further described in section 2.1 and Paper VI. The conductivity, on the other hand, is largely dominated by Coulomb scattering between charge carriers and benefits from the absence of Coulomb interactions. The magnitude of these effects scales with the disorder of the unperturbed DOS leading to a spread and thus a non-universal $S \propto \ln (\sigma)$ relationship for materials of different disorder seen on the left side of Figure 4.9. The spread between different materials is also observed in literature, seen on the right side of Figure 4.9. Note that this contrasts with the material independent power-law relationship $S \propto \sigma^{-\frac{1}{4}}$ relationship for doped polymers described in section 3.3.6. A practical implication of this finding is that OFETs are, in general, not a suitable testbed for thermoelectric investigations of doped ODSCs.
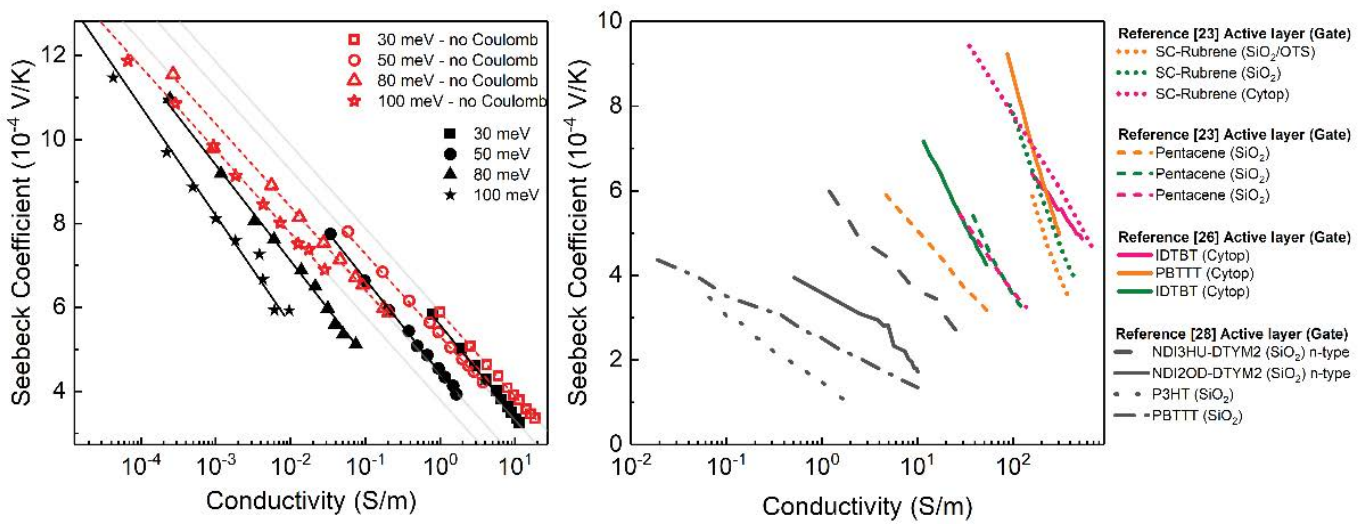

Figure 4.9 (left) Seebeck coefficient as a function of conductivity calculated with the Monte Carlo algorithm described in section 5. Black filled symbols are calculated including full Coulomb interactions, while calculation of the red open symbols excluded Coulomb interactions. Solid lines are guides to the eye. (right) Compilation of experimental literature data, from references indicated in the legend, shows a large spread in inclination and position in the $S$ vs. $\sigma$ plot. 


\subsection{BIBLIOGRAPHY}

1. Zhang, W. et al. Indacenodithiophene semiconducting polymers for high performance air stable transistors Indacenodithiophene semiconducting polymers for high performance air stable transistors. J. Am. Chem. Soc. 132, 11437 (2010).

2. Zhang, X. et al. Molecular origin of high field-effect mobility in an indacenodithiophene-benzothiadiazole copolymer. Nat. Commun. 4, 1-9 (2013).

3. Kim, G. et al. A thienoisoindigo-naphthalene polymer with ultrahigh mobility of $14.4 \mathrm{~cm} 2 / V$ ??s that substantially exceeds benchmark values for amorphous silicon semiconductors. J. Am. Chem. Soc. 136, 9477-9483 (2014).

4. Chen, Z. et al. High-Performance Ambipolar Diketopyrrolopyrrole-Thieno[3,2b ]thiophene Copolymer Field-Effect Transistors with Balanced Hole and Electron Mobilities. Adv. Mater. 24, 647-652 (2012).

5. Shimotani, H., Asanuma, H., Takeya, J. \& Iwasa, Y. Electrolyte-gated charge accumulation in organic single crystals. Appl. Phys. Lett. 89, (2006).

6. Yuen, J. D. et al. Electrochemical doping in electrolyte-gated polymer transistors. J. Am. Chem. Soc. 129, 14367-14371 (2007).

7. Xia, Y., Xie, W., Ruden, P. P. \& Frisbie, C. D. Carrier Localization on Surfaces of Organic Semiconductors Gated with Electrolytes. Phys. Rev. Lett. 105, 36802 (2010).

8. Horowitz, G., Hajlaoui, R. \& Delannoy, P. Temperature Dependence of the Field-Effect Mobility of Sexithiophene. Determination of the Density of Traps. J. Phys. III 5, 355-371 (1995).

9. Salleo, A. et al. Intrinsic hole mobility and trapping in a regioregular poly(thiophene). Phys. Rev. B 70, 115311 (2004).

10. Brown, a. R., Jarrett, C. P., de Leeuw, D. M. \& Matters, M. Field-effect transistors made from solution-processed organic semiconductors. Synth. Met. 88, 37-55 (1997). 
11. Österbacka, R., An, C. P., Jiang, X. M. \& Vardeny, Z. V. Two-Dimensional Electronic Excitations in Self-Assembled Conjugated Polymer Nanocrystals. Science (80-. ). 287, 839-842 (2000).

12. Vissenberg, M. C. J. M. \& Matters, M. Theory of the field-effect mobility in amorphous organic transistors. Phys. Rev. B 57, 13 (1998).

13. Brondijk, J. J. et al. Two-dimensional charge transport in disordered organic semiconductors. Phys. Rev. Lett. 109, 1-5 (2012).

14. Kronemeijer, A. J. et al. Two-dimensional carrier distribution in top-gate polymer field-effect transistors: Correlation between width of density of localized states and urbach energy. Adv. Mater. 26, 728-733 (2014).

15. Dinelli, F. et al. Spatially Correlated Charge Transport in Organic Thin Film Transistors. Phys. Rev. Lett. 92, 116802 (2004).

16. Fabiano, S. et al. From monolayer to multilayer n-channel polymeric fieldeffect transistors with precise conformational order. Adv. Mater. 24, 951-956 (2012).

17. Granstrom, E. L. \& Frisbie, C. D. Field effect conductance measurements on thin crystals of sexithiophene. J. Phys. Chem. B 103, 8842-8849 (1999).

18. Muck, T. et al. In situ electrical characterization of DH4T field-effect transistors. Synth. Met. 146, 317-320 (2004).

19. Janasz, L. et al. Microstructure-Dependent Charge Carrier Transport of Poly(3hexylthiophene) Ultrathin Films with Different Thicknesses. Langmuir 33, 4189-4197 (2017).

20. Zaumseil, J. \& Sirringhaus, H. Electron and ambipolar transport in organic fieldeffect transistors. Chem. Rev. 107, 1296-1323 (2007).

21. Tanase, C., Meijer, E. J., Blom, P. W. M. \& De Leeuw, D. M. Unification of the hole transport in polymeric field-effect transistors and light-emitting diodes. Phys. Rev. Lett. 91, 216601 (2003).

22. Sirringhaus, H. Device physics of solution-processed organic field-effect transistors. Adv. Mater. 17, 2411-2425 (2005). 
23. Veres, J., Ogier, S. D., Leeming, S. W., Cupertino, D. C. \& Khaffaf, S. M. Low-k insulators as the choice of dielectrics in organic field-effect transistors. Adv. Funct. Mater. 13, 199-204 (2003).

24. Coehoorn, R., Pasveer, W., Bobbert, P. \& Michels, M. Charge-carrier concentration dependence of the hopping mobility in organic materials with Gaussian disorder. Phys. Rev. B 72, 155206 (2005).

25. Di Pietro, R. et al. Coulomb Enhanced Charge Transport in Semicrystalline Polymer Semiconductors. Adv. Funct. Mater. 26, 8011-8022 (2016).

26. Von Mühlenen, A., Errien, N., Schaer, M., Bussac, M. N. \& Zuppiroli, L. Thermopower measurements on pentacene transistors. Phys. Rev. $B$ Condens. Matter Mater. Phys. 75, 3-8 (2007).

27. Pernstich, K. P., Rössner, B. \& Batlogg, B. Field-effect-modulated Seebeck coefficient in organic semiconductors. Nat. Mater. 7, 321-325 (2008).

28. McCulloch, I. et al. Liquid-crystalline semiconducting polymers with high charge-carrier mobility. Nat. Mater. 5, 328-333 (2006).

29. Venkateshvaran, D. et al. Approaching disorder-free transport in high-mobility conjugated polymers. Nature 515, 384-388 (2014).

30. Venkateshvaran, D., Broch, K., Warwick, C. N. \& Sirringhaus, H. Thermoelectric transport properties of high mobility organic semiconductors. in (eds.

McCulloch, I. \& Jurchescu, O. D.) 9943, 99430 U (2016).

31. Zhang, F. et al. Modulated Thermoelectric Properties of Organic Semiconductors Using Field-Effect Transistors. Adv. Funct. Mater. 25, 30043012 (2015).

32. Kaiser, A. B. Electronic transport properties of conducting polymers and carbon nanotubes. Reports Prog. Phys. 64, 1 (2001).

33. Glaudell, A. M., Cochran, J. E., Patel, S. N. \& Chabinyc, M. L. Impact of the Doping Method on Conductivity and Thermopower in Semiconducting Polythiophenes. Adv. Energy Mater. 5, 1401072 (2015).

34. Xuan, Y. et al. Thermoelectric properties of conducting polymers: The case of poly(3-hexylthiophene). Phys. Rev. B 82, (2010). 


\section{Kinetic Monte CARLo AlgoRItHM}

In all Papers of this thesis, with the exception of Paper III, we used a kinetic Monte Carlo (MC) algorithm developed by Martijn Kemerink to simulate charge transport by thermally activated nearest-neighbour-hopping on a regular lattice ${ }^{1-3}$. The MC algorithm has been positively benchmarked against a wide variety of applications, ranging from solar cells to high concentration OFETs and has continuously proven to be a valuable tool in the investigation of transport, energetics and position of charge carriers in organic semiconductors ${ }^{4,5}$.

While there are many input parameters to the simulations, only surprisingly few have a significant influence on the calculation output (within reasonable limits). Within the context of this thesis, the three basic physical principles that govern movement of the simulated charge carriers are:

- Nearest neighbour hopping on a regular lattice

- Energetically disordered hopping sites

- Full Coulomb interaction between all charges and influence on site energies

Hence, throughout the work in this thesis the varied parameters are related to either of these three concepts and centre around

- Attempt-to-hop frequency and electric field

- The shape and width of the disorder

- Dielectric constant, inter-site distance and charge carrier concentration

These simple assumptions are sufficient to coherently investigate charge and energy transport. The detailed workings the used kinetic $M C$ technique are described in the following 


\subsection{GeOMetries ANd Hopping MeChANISM}

In principle, our kMC simulations mimic real-world experiments, with the added benefit of being able to control every aspect of the virtual sample. However, to facilitate reasonable computation times, the sample to be simulated must be simplified and translated to a mathematical model. In the present case the biggest simplification is the assumption of hopping sites being arranged on a regular lattice depicted in Figure 5.1 with a unit cell of volume

$$
V_{U C}=\left(a_{N N x} \times a_{N N y} \times a_{N N Z}\right),
$$

rather than following the disordered morphology of many ODSCs. The lattice constants in $x_{-}^{-}, y^{-}$, and $z$-direction are $a_{N N x}, a_{N N y}$ and $a_{N N Z}$, respectively. However, the influence of the morphology mostly plays out in energetic disorder and furthermore, the latter is dominating charge transport as has been extensively discussed throughout the thesis. This makes the assumption of a regular lattice a reasonable assumption. The size of the virtual sample (box size) is determined by the number of unit cells in $x-, y$-, and $z$-direction. Throughout the work of this thesis it was assured that the box size was large enough to not create size-related artefacts in the output of the simulations. However, in view of higher calculation speed a smaller box is desirable.

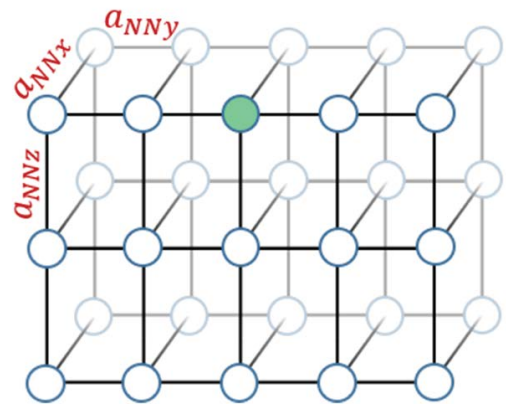

Figure 5.1 Geometric relations of a rectangular lattice model as used in e.g. Papers IV, $V$ and $\mathbf{V I}$.

Initially, site energies $E$ are distributed randomly according to either a Gaussian, exponential or constant distribution function (see Equations (2.2) and (2.3)). Transition rates between sites $i$ and $j$ are calculated by the Miller Abrahams expression (Equation (2.13)) and since hopping in the algorithm is limited to nearest 
neighbour sites, the hopping distance $\vec{r}_{i j}$ is constant and so is the first exponent in Equation (2.13) and it follows

$$
\vartheta_{i j}=\vartheta_{0}^{*} \begin{cases}\exp \left(-\frac{E_{j}-E_{i}-q \vec{r}_{i j} \vec{F}}{k_{B} T}\right) & E_{j}>E_{i} \\ 1 & E_{j} \leq E_{i} .\end{cases}
$$

To induce directed hopping and thereby net charge flow, an electric field $\vec{F}$ is applied. When simulating FETs, a field in the $z$-direction is applied that populates the system with charge carriers according to Equation (4.1). As discussed in section 2.2.3, the term $q \vec{r}_{i j} \vec{F}$ accounts for the increased hopping rate in the direction of the field. In the case of a non-cubic lattice, such as in Figure 5.1, hopping rates are modified by the ratio $a_{d} / a_{0}$ between $a_{d}$ the lattice constant in the direction of the hop and $a_{0}$ a reference lattice constant according to

$$
\vartheta_{d} \propto \exp \left(-\frac{2\left(a_{d}-a_{0}\right)}{\alpha}\right)
$$

with $\alpha$ the localization length (see section 2.2.1). The choice of which of the 6 possible hops actually occurs follows a random selection process with the hopping rate used as a weighing factor. The time $\tau$ between two hopping events is calculated as

$$
\tau=-\frac{\ln (\varsigma)}{\sum \vartheta}
$$

where $\varsigma$ is a randomly generated number in the interval ]0,1[ and the denominator is the sum of all possible hopping rates. The movement of charges is tracked individually and the sum of transported charge $q$ over a distance $\Delta r$ per time $t$ gives the current density in the direction of $r$ as a function of time

$$
j(t)=\frac{d}{d t}\left[\frac{1}{A L} \sum_{k} q \Delta r_{k}\right],
$$

with $\mathrm{A}$ the cross section of the simulation box and $L$ the length of the box along which the current is calculated.

The charge carriers are initially distributed at random energies and positions before thermalizing to their thermal equilibrium positions and energies by penalty-free hops to lower energies. This process was used in Paper I to find an empirical expression for 
the relaxation rate of the effective temperature $T_{\text {eff }}$ (see also sections 2.3 .3 and 2.3.4). Hence, the current at small times is high and "relaxes" to a constant value at larger times. Since this thesis considers equilibrium charge transport (except Paper I) convergence of the current density was assured in all cases before the calculation of transport parameters. The mobility is obtained from the drift equation

$$
\mu=\frac{q n F}{j} \text {. }
$$




\subsection{Calculation of Density of States and Density of Occupied States}

As was mentioned multiple times in previous sections, the DOS is heavily influenced by the Coulomb potential of charge carriers. This is especially true at high concentrations, the regime which this thesis mostly deals with, and hence Coulomb interactions must be considered in the $\mathrm{MC}$ algorithm. Coulomb interactions are included between all charge carriers and their corresponding twin charges due to periodic boundary conditions at each end of the box up to a cut-off length of 5 box sizes. In the case of a FET structure (Papers IV, $\mathbf{V}$ and $\mathbf{V I}$ ) the interaction of charges with their image charges due to the gate contact and dielectric contrast between the semiconductor layer and the gate dielectric are included as well.

The energy $E_{\text {site }}^{i}$ of a carrier occupying site $i$ and Coulombically interacting with all $N$ other charge carriers is calculated as the sum of the site energy $E_{i}$ of the input distribution, and the Coulomb energy between the two charges as

$$
E_{\text {site }}^{i}=E_{i}+\sum_{j \neq i}^{N}-\frac{q}{4 \pi \varepsilon_{0} \varepsilon_{r} R_{i j}}=E_{i}+\sum_{j \neq i}^{N} \mathrm{E}_{\mathrm{C}}^{i, j}
$$

where $R_{i j}$ is the distance between two charge carriers. To avoid the problem of divergence in the Coulomb term on the right-hand side of Equation (5.6) when two charges occupy the same site, $E_{C}$ is truncated at an energy $E_{B}$, which serves the function of an on-site barrier to be overcome for multiple occupation. Throughout this thesis $E_{B}$ was chosen to be $0.5 \mathrm{eV}$ which is comparable to the binding energy of a Frenkel exciton in organic semiconductors. In Paper VI the effect of the on-site barrier on multiple occupation and charge transport was investigated and $E_{B}$ was varied between $0.05 \mathrm{eV}, 0.1 \mathrm{eV}$ and $0.5 \mathrm{eV}$.

The same energy modification applies to all sites a charge carrier can move to and so also the DOS seen by the charge $i$ is modified by the Coulomb potentials of all other charge carriers. For this, the final site energies $E_{\text {site, } f}^{i}$ as experienced by a carrier on the initial site $i$ with energy $E_{\text {site }}^{i}$ by hopping to all other sites $f$, is calculated in analogy to Equation (5.6)

$$
E_{\text {site }, f}^{i}=E_{f}+\sum_{j \neq i}^{N} E_{C}^{f, j} .
$$


All these changes are implicitly included in the calculation of the hopping rate via the term $E_{j}-E_{i}$ in Equation (5.1) and have therefore also significant influence on charge transport. After every hopping event all Coulomb energies are updated to reflect the new situation after the hop. Due to the interdependencies between the current position of a charge and its next position, also the DOS experiences a thermalisation process, which needs to be completed before an analysis can be made. The DOS is calculated as explained in section 2.1 by creating a histogram of all final site energies $E_{\text {site }, f}^{i}$ averaged over all particles in the simulation and moving to all possible sites in the simulation box. Analogously, the DOOS is the histogram of the site energies $E_{\text {site }}^{i}$ of all $N$ particles.

The Fermi level $E_{F}$ and the effective temperature $T_{\text {eff }}$ are obtained by fitting the Fermi-Dirac distribution to the quotient of DOOS and DOS (see also Figure 2.22). As described in Paper I, the value of $T_{e f f}$ can deviate significantly from the lattice temperature at high electric fields.

In Paper VI we show that significant multiple occupation of individual sites ( $>2$ charges per site) can occur already at low concentrations if the on-site barrier is small compared to the typical Coulomb energy between charges. To retain meaning of the Fermi level a hopping site is added in energy space to the total DOS for every occupied site of the empty DOS, with an energy according to Equations (5.6) and (5.7) plus a shift by $E_{B}$ towards higher energies. This ensures the per-site occupation remains below-or-equal to 1 and Fermi-Dirac statistics are valid, allowing the calculation of meaningful Fermi levels. Since we place no restrictions on the number of charge carriers that can occupy an individual site in space, our MC algorithm does not treat the particles as Fermions with spin but rather as classical particles. Multiple occupation is then paramount to filling up a large number of sub-sites, which are close in energy space compared to the other energy scales in the system. 


\subsection{THERMOELECTRIC COEFFICIENTS}

Seebeck coefficient is calculated according to the general expressions in Equations (3.18) where the transport energy $E^{*}$ is accordingly calculated as

$$
E^{*}=\frac{\int E \sigma(E) d E}{\int d E \sigma(E)} .
$$

The electrical conductivity is calculated as the quotient of the current density $j(E)$ and the electric field $F$,

$$
\sigma(E)=\frac{j(E)}{F} .
$$




\subsection{BIBLIOGRAPHY}

1. Schönherr, G., Bässler, H. \& Silver, M. Dispersive hopping transport via sites having a Gaussian distribution of energies. Philos. Mag. B 44, 47-61 (1981).

2. Bassler, H. Charge Transport in Disordered Organic Photoconductors. Phys. Status. Solidi. B, Basic Res. 175, (1993).

3. Greenham, N. C. \& Bobbert, P. A. Two-dimensional electron-hole capture in a disordered hopping system. Phys. Rev. B 68, 245301 (2003).

4. Zuo, G., Liu, X., Fahlman, M. \& Kemerink, M. High Seebeck Coefficient in Mixtures of Conjugated Polymers. Adv. Funct. Mater. 1703280, 1703280 (2017).

5. Melianas, A. Non-Equilibrium Charge Motion in Organic Solar Cells. (2017). 


\section{EXPERIMENTAL TECHNIQUES}

This chapter briefly describes the experimental techniques used to measure conductivity, mobility and Seebeck coefficient. Numerical methods are described in section 5 and analytical models are introduced in sections 2-4. All papers also include a section on the methods and materials used.

\subsection{Electrical Characterisation}

Room temperature measurements of the conductivity for Paper II were obtained in air while in Paper III all samples were measured in a glove box under dry nitrogen atmosphere. Voltage $V$ and current $I$ are recorded during a voltage sweep in a Kelvin (4 point) arrangement. The conductivity is then calculated from the device geometry and the thickness of the conductive channel $t_{C}$, measured with a profilometer, by

$$
\sigma=\frac{L_{C}}{t_{C} W_{C}} \frac{I}{V},
$$

where $W_{C}$ and $L_{C}$ are the width and length of the channel, respectively. Typical film thicknesses are in the $100 \mathrm{~nm}$ range and thus the assumption that charge transport takes place through the whole sample is valid.

To obtain the temperature dependence of the electrical conductivity for Papers I and III, the previously described measurement was done in a temperature-controlled vacuum probe station, where good thermal contact was ensured by application of a thin layer of thermal paste. After settling of the temperature after every temperature step, the sample was allowed to thermalize for 5-10 minutes before a measurement was taken.

The electrical characterization of OFETs in Papers IV and $\mathbf{V}$ was done externally by Simone Fabiano and follows the methods described in sections 4.1 and 4.2. 


\subsection{Determination of the SeebeCk Coefficient}

Experimentally, the Seebeck coefficient $S$ is obtained per Equation (3.3) by measuring the thermovoltage $V_{t h}$ and the temperature difference $\Delta T$

$$
S=\frac{V_{t h}}{\Delta T} .
$$

$V_{t h}$ can either be measured directly upon applying a temperature gradient between two contacts and measuring the voltage that arises between them or, as was done in Paper III by successive current-voltage (IV) measurements, shown in Figure 6.1 and described in the following. The latter method has the added benefit of removing constant offsets from the measurement system.
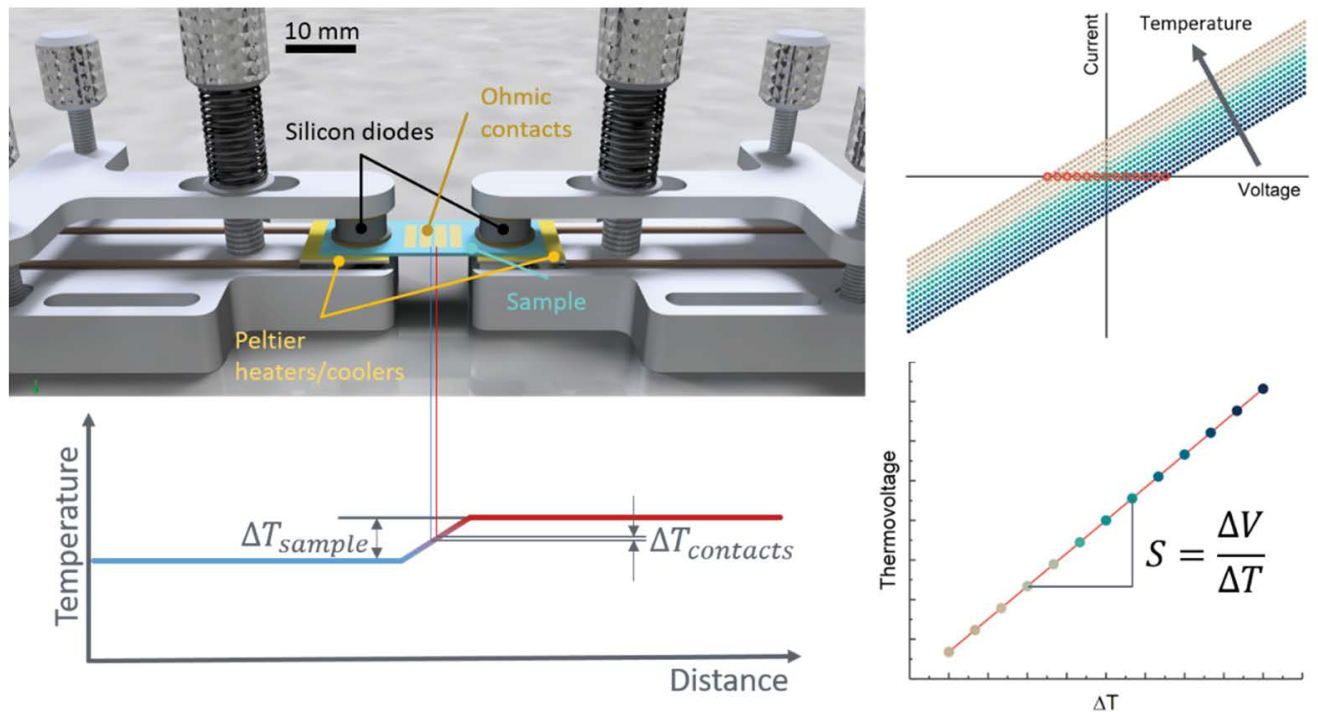

Figure 6.1 (top left) Rendering of the Seebeck measurement setup. (bottom left) Temperature gradient along the sample and between electrical contacts. (top right) Current-voltage characteristics obtained at different temperatures as indicated by the colours. Thermovoltages are indicated by the red circles. (bottom right) Thermovoltage plotted as a function of temperature difference between contacts. The inclination corresponds to the Seebeck coefficient.

The sample is sandwiched between Peltier elements and silicon temperature sensors. While one Peltier element heats up the other cools down according to the sign of the current applied to them. This creates a temperature gradient along the sample and 
the temperature difference between the ohmic contacts can be calculated from the geometric proportionalities, assuming a linear temperature gradient as shown in the left part of Figure 6.1. After the temperature at the temperature sensors has settled and the sample had time to fully thermalize (typically 300 seconds) an IV measurement is taken and the whole process is repeated for different temperature differences.

After a number of IV curves have been taken at different temperatures, seen in the top right part of Figure 6.1, the intercepts with the voltage axis are determined from linear fits to the IV data. These intercepts correspond to the thermovoltages and if plotted against $\Delta T$ and fitted with a linear model, give the Seebeck coefficient as the inclination of the linear fit as can be seen in the bottom right part of Figure 6.1.

To suppress the creation of a thermovoltage between the ohmic contacts and the probe needles, both were chosen to be of the same material, namely gold. However, no correction has been made to account for the possibility of a thermovoltage between the sample material and the contacts, due to the relatively small Seebeck coefficient of gold $(1.5 \mu \mathrm{V} / \mathrm{K})$ compared to the materials studied in this thesis ( $>30$ $\mu \mathrm{V} / \mathrm{K})$.

Figure 6.2 shows a picture of the setup used in Paper III for room temperature measurements under Nitrogen atmosphere. The overall sample length was $27 \mathrm{~mm}$, with a channel length $L_{C}=0.5 \mathrm{~mm}$ and a channel width $W_{C}=7 \mathrm{~mm}$. The small channel length was needed due to the small conductivities for most samples measured in Paper III. The temperature differences between the two contacts was varied from $0 \mathrm{~K}$ to around $\pm 1 \mathrm{~K}$. For temperature dependent measurements a smaller setup was used, pictured in Figure 6.3, to be able to accommodate the setup in the vacuum probe station. 


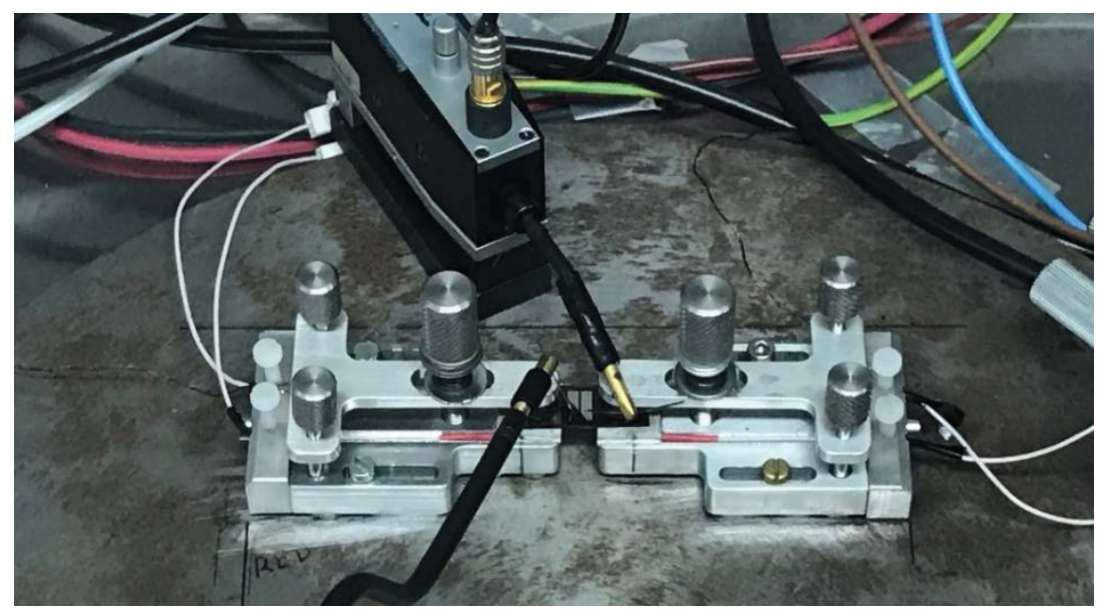

Figure 6.2 Photograph of the setup used to measure the Seebeck coefficient in Paper III at room temperature under nitrogen atmosphere.

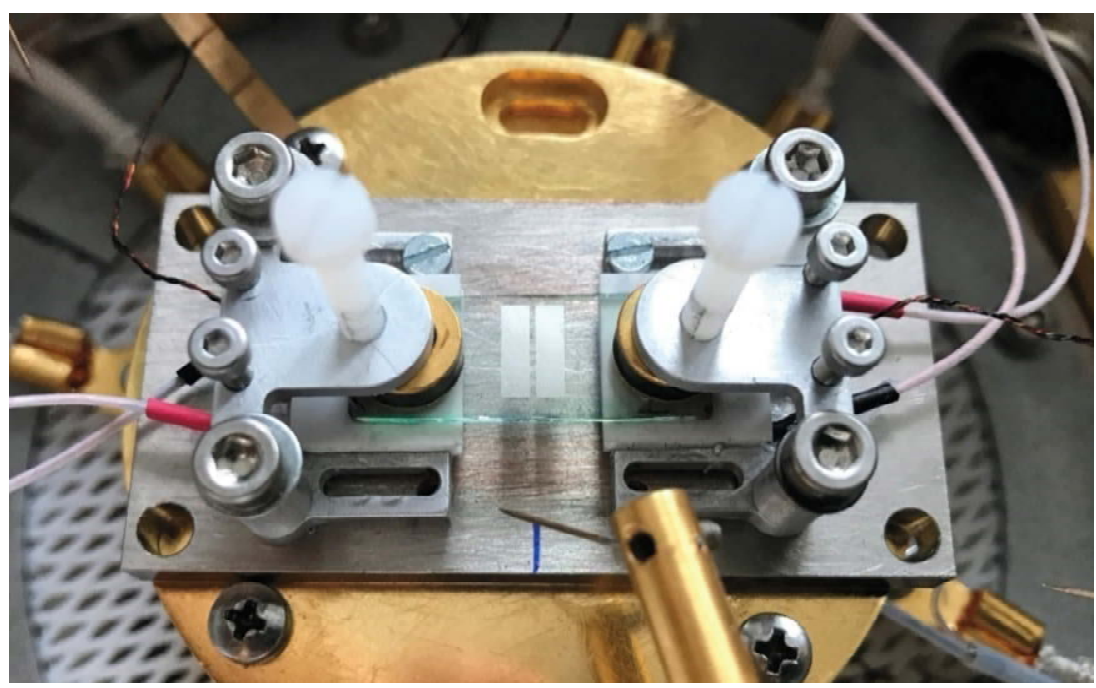

Figure 6.3 Setup used for temperature dependence measurement of the Seebeck coefficient in the vacuum probe station. 


\subsection{Determination of the Seebeck Coefficient of OFETS}

The Seebeck coefficient of OFETs in Paper $\mathbf{V}$ were measured by Simone Fabiano in the laboratory of organic electronics at the Norrköping campus of Linköping University. The measurements follow the same principle as described above for the thin film samples and follows the measurement and calibration routines described by Venkateshvaran et al. in Ref. ${ }^{1}$. The experimental setup is schematically shown in Figure 6.4 with a channel length $L_{C}$ between 20-50 $\mu \mathrm{m}$ and channel width $W_{C}=1000$ $\mu \mathrm{m}$. Due to the small length scales involved, the source and drain contacts act also as temperature sensors. After calibration, the change in resistance of these gold contacts indicates their temperature. All contacts have a width of $20 \mu \mathrm{m}$. The heater is another gold strip separated from the closest contact by a distance of $20 \mu \mathrm{m}$, which heats the sample via resistive heating upon application of a current. The active layer and gate electrode do not overlap with the heating wire to avoid cross-talk between them.

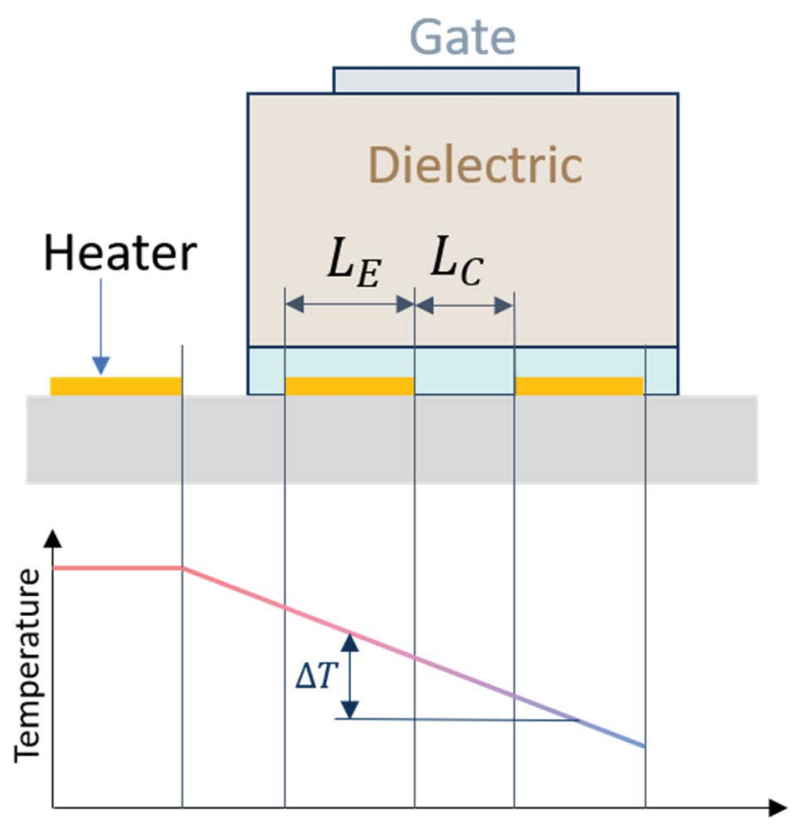

Figure 6.4 Experimental setup to measure the Seebeck coefficient of OFETs at the laboratory of organic electronics at the Norrköping campus. 


\subsection{BIBLIOGRAPHY}

1. Venkateshvaran, D. et al. Approaching disorder-free transport in high-mobility conjugated polymers. Nature 515, 384-388 (2014). 


\section{CONCLUSION}

The main conclusion to be taken away from this thesis is that the broadened DOS that arises from the disordered nature of ODSCs and is so characteristic for the field of organic electronics, is not constant with charge carrier concentration, regardless of doping mechanism. Further broadening and modifications of the shape of the DOS occur with increasing concentration and, importantly, the changes to the DOS and the increasing inter-carrier Coulomb interactions have a noticeable effect on charge and energy transport and become dominant at high concentrations.

Due to these changes, the resulting conductivity, mobility and Seebeck coefficient behaviour cannot be explained by "classical" models that assume an unvarying DOS but require the inclusion of the Coulomb-induced effects, such as described in this thesis. The intricacies of these effects are rather complex and implicit in their nature and thus hard to infer just from experimental measurements alone, but insights can be gained by adequate numerical methods that account for the dominant effects, such as the kinetic Monte Carlo algorithm used in this thesis. Predictions from numerical simulations can also be used to test analytical models and serve as a starting point for new experimental investigations, based on interesting phenomena observed numerically.

The work of this thesis also raises new questions and promotes further investigations of the high-carrier density regime.

One example is the still unexplained conductivity - Seebeck coefficient behaviour of PEDOT derivatives, which generally do not follow the empirical trend observed by nearly all other doped polymer systems. This is especially interesting since PEDOT:PSS and PEDOT:Tos are considered to be the most promising polymer materials for p-type thermoelectric applications, exhibiting high power factors and good mechanical and chemical stability.

Another topic of further investigation would be the Seebeck coefficient of organic field-effect transistors at high charge carrier concentrations. Numerically, we expect a sign change of the Seebeck coefficient at much lower concentrations than expected from other models, due to the Coulomb-induced changes of the DOS. However, no such investigations have been published to date. 


\section{Chemical AbBreViations}

\section{[(EMIM) ${ }^{+}($TFSI) $]$}

1-ethyl-3-methylimidazoliumbis(trifluoromethylsulfonyl)imide

\section{DMF}

dimethyl formamide

\section{DMSO}

dimethyl sulfoxide

\section{DPPTTT}

poly-[2,5-bis(2-octyldodecyl)-3,6-di(thiophen-2-yl)pyrrolo[3,4-c]pyrrole-1,4(2H,5H)dionel-alt-thieno[3,2-b]thiophene

\section{$\mathrm{F}_{4} \mathrm{TCNQ}$}

2,3,5,6-tetrafluoro- 7,7,8,8-tetracyanoquinodimethane

\section{IDTBT}

indacenodithiophene-co-benzothiadiazole

$\mathrm{MDMO}=\mathrm{OC}_{1} \mathrm{C}_{10}-\mathrm{PPV}$

poly(2-methoxy-5-(30; 70-dimethyloctyloxy)-p-phenylene vinylene)

\section{P(NDI2OD-T2)}

poly\{[N, $N^{\prime}$-bis(2- octyldodecyl)-naphthalene-1,4,5,8-bis(dicarboximide)-2,6-diyl]-alt5,5'-(2,2'-bithiophene)\}

\section{P3HT}

poly(3-hexylthiophene)

\section{PBTTT}

Poly(2,5-bis(3- $n$-alkyl-2yl)thieno[3,2- $b$ ] thiophene)

\section{PEDOT}

poly(3,4-ethylenedioxythiophene)

$\mathrm{PF}_{6-}$

Hexafluorophosphate

\section{PMMA}

poly methyl-methacrylate

PPV

Polyphenylenevinylene

\section{PQT-12}

Poly[5,5 '-bis(3-dodecyl-2-thienyl)-2,2' -bithiophene] 


\section{PSS}

poly(4-styrenesulfonate)

PTV

poly (2, 5-thienylene vinylene)

T6

sexithiophene

THF

tetrahydrofuran

Tos

tosylate

VOPc

vanadyl-phthalocyanine 



\section{ACKNOWLEDGEMENTS}

I sincerely hope that I have sufficiently acknowledged the people that I am indebted to during the 4 years of this thesis verbally, but in any case, I would like to mention them here in writing.

The major contribution to the creation of this thesis and the completion of my PhD comes from my supervisor Martijn Kemerink who has guided my relentlessly out of the self-dug pits of unimportant details, destructive self-criticism and obsessive comma placement and back onto the road of scientific reasoning, deduction and investigation. I am especially grateful for the sheer endless amount of patience and goodwill he has shown me and amazed by the incredible depth and variety of his knowledge, extending far beyond physics.

Without Armantas Melianas I would not have entered the PhD journey and would not be writing any of these words since he encouraged me to apply with and introduced me to Martijn. He also introduced me to the scientific practices and techniques and offered solace to my PhD worries by sharing his insights and own experiences. He is an amazing scientist and teacher and someone to look up to (however, he failed to teach me a 360 flip).

Great many thanks are also due to Simone Fabiano for fabricating, measuring and analysing the organic field-effect transistors that provided the experimental basis for our numerical investigations and Kevin van de Ruit for supplying me with a wealth of experimental data on PEDOT to start my PhD. To Liangqi Ouyang I am grateful for the large amount of PEDOT samples in all kinds of variations he has supplied me with.

Thanks to our administrators Jenny Äh/ström and Anna-Maria Uhlin, all the paperwork that comes with working, travelling and graduating was never a problem and easily handled. I would also like to thank IFM's lab technician Jörgen Bengtsson for his help and support with building and fixing various lab equipment.

The sometimes-frustrating moments in the laboratory or in front of the computer were made enjoyable by my group colleagues Nikolaos Felekidis, Guangzheng Zuo, 
Olof Andersson, Indré Urbanavičiūtè, Tim Cornelissen and Davood Abbaszadeh. I would especially like to thank Guangzheng for his collaborations with me and incredible number of high-quality samples he produced and patiently explaining me the basic concepts of chemistry. Thank you, Nikolaos, Olof and Guangzheng for the fun times in Russia, China, France, Greece and Sweden and your help in finding my way back to the hotel.

Luncheon was always a highlight of the day not only because of sustenance but also because it meant meeting some fun and interesting people including Riccardo Volpi, Rafael Sanchez, Arnaud le Febvrier, Davide Gambino (lead vocals), Víctor Gervilla Palomar (guitar and supporting vocals), Andreas Jamnig, Ivan Yakimenko, Laurent Souqui, Claudia Schnitter and Tim Cornelissen. Without you guys life would have been infinitely duller, not only during lunchtime.

Without the constant encouragement, motivation, support and love from my girlfriend I would have given up a long time ago. Thank you, Anna-Giulia Scaglia and thank also to all other Scaglias.

Finally, I thank my family for the constant support they have given me and especially my mother. Danke für Alles Mutti! 


\section{Papers}

The papers associated with this thesis have been removed for copyright reasons. For more details about these see:

http://urn.kb.se/resolve?urn=urn:nbn:se:liu:diva-145673 